\title{
Estimation of spatial autoregressions with stochastic weight matrices
}

\author{
Abhimanyu Gupta* \\ Department of Economics \\ University of Essex
}

March 15, 2018

\begin{abstract}
We examine a higher-order spatial autoregressive model with stochastic, but exogenous, spatial weight matrices. Allowing a general spatial linear process form for the disturbances that permits many common types of error specifications as well as potential 'long memory', we provide sufficient conditions for consistency and asymptotic normality of instrumental variables, ordinary least squares and pseudo maximum likelihood estimates. The implications of popular weight matrix normalizations and structures for our theoretical conditions are discussed. A set of Monte Carlo simulations examines the behaviour of the estimates in a variety of situations. Our results are especially pertinent in situations where spatial weights are functions of stochastic economic variables, and this type of setting is also studied in our simulations.
\end{abstract}

JEL classifications: C21, C31, C36

Keywords: Spatial autoregression; stochastic spatial weights; spatial linear process; instrumental variables; ordinary least squares; Gaussian pseudo maximum likelihood

*Email: a.gupta@essex.ac.uk. 


\section{Introduction}

Spatial autoregressive (SAR) models, due to Cliff and Ord (1973), have recently become very popular in applied and theoretical research. Their main feature is the modelling of spatial dependence by allowing a form of direct interaction between units through spatial weight matrices $W_{j}, j=1, \ldots, p$. The elements of $W_{j}$ measure distance between units, which may be geographic but in general can be (inverse) economic distances.

While the majority of the literature on estimation and inference for SAR models, e.g. Kelejian and Prucha (1998, 1999, 2001), Lee (2002, 2003, 2004), Robinson (2010), Lee and Liu (2010), Su and Jin (2010), Lee and $\mathrm{Yu}$ (2013), Gupta and Robinson (2015), has assumed the $W_{j}$ to be deterministic, examples abound that imply stochastically generated $W_{j}$. Most commonly a typical element of $W_{j}$ is determined by economic variables that may themselves be stochastic. Conley and Ligon (2002) study cross-country spillovers in long-run growth rates using several distance measures. While one of them, geographic distance, is evidently fixed, the other two measures, United Parcel Service shipping costs and airfare, are more difficult to justify to be fixed in repeated sampling. Both, at the very least, are subject to random shocks in the economic conditions of each pair of countries, among many other factors. Conley and Dupor (2003) take input-output relations as a measure of economic distance, and it is reasonable to imagine that these relations are stochastic and not fixed. In Yuzefovich (2003) spatial weight matrices are constructed using a variety of economic distances, e.g. trade between two countries and competition in borrowing from a common lender. These variables would generally be considered stochastic in econometric analyses that use such data. Another example is Baltagi, Fingleton, and Pirotte (2014), who construct a weight matrix using commuting frequencies between districts in the UK. Commuting frequencies between two districts depend heavily on macro and microeconomic factors that are stochastic, and therefore may be anticipated to be stochastic too. Souza (2015) considers a SAR model in which networks may form stochastically, captured by nonzero spatial weight matrix elements. However he treats these elements as unobserved heterogeneity, and hence unknown, whereas in our treatment they are known. Robinson (2008) discusses a SAR with stochastic weights in the context of correlation testing. In a recent survey, Boucher and Fortin (2016) discuss the concept of stochastic spatial weight matrices from the perspective of impact caused by a policy shock. They stress that social interaction models based on a stochastic network structure, such as the SAR model considered in this paper, should include a description of the network formation process, and that researchers should discuss the impact of proposed policy shocks on the assumed network.

In this paper we will justify instrumental variables (IV), ordinary least squares (OLS) and pseudo maximum likelihood estimates (PMLE) with stochastic but exogenous $W_{j}$. Asymptotic theory for IV estimates of SAR model parameters was introduced first in Kelejian and Prucha (1998), and subsequently also studied by Lee (2003). IV is employed because of endogeneity problems, but Lee (2002) demonstrated that OLS can deliver consistent and asymptotically normal estimates of SAR model parameters under certain circumstances, thus correcting a tendency to casually discard OLS as a suitable method for SAR estimation and inference. A more general treatment by Gupta and Robinson (2015) examined IV and OLS estimates for an increasing order version of the SAR model, but with iid disturbances. For PMLE, Lee (2004) developed asymptotic theory for SAR models with a single weight matrix, while Gupta and Robinson (2018) consider SAR models in which both the number of weight matrices and regressors can diverge with sample size. Both papers take independent 
disturbances that are also identically distributed in the case of Lee (2004) but need not be in Gupta and Robinson (2018). On the other hand, Delgado and Robinson (2015) provide asymptotic theory for estimation of spatial models of a rather general type, including SAR, spatial moving average (SMA) and spatial ARMA, allowing a linear process type disturbance structure for their distributional results.

Theory has been developed for estimation with endogenous $W_{j}$. Kelejian and Piras (2014) consider such a model and develop IV type estimates. Qu and Lee (2015) were critical of their restrictive assumptions, and instead use the near epoch dependence (NED) theory of Jenish and Prucha (2012) to establish consistency and asymptotic normality of estimates in a more general setting. Building on the spatial martingale limit theory in Kuersteiner and Prucha (2013), endogenous weight matrices are also permitted in a generalized method of moments framework in Kuersteiner and Prucha (2015). However the intermediate case, with stochastic but exogenous $W_{j}$ has received little theoretical attention. This case can cover situations of economic interest where spatial weights are generated by exogenous regressors, and can be examined in a very general framework that does not require NED process theory. For observations recorded at locations $r$ and $s$ the latter essentially requires the locations to be geographic (in the sense that they are in Euclidean space) due to a notion of dependence reducing as the distance between $r$ and $s$ increases. Thus it is not generally applicable to data whose locations do not have a geographic interpretation. On the other hand, the SAR model has been considered to be particularly appealing because of its ability to handle data in general economic spaces, such as income space, where geographical interpretations may not be natural and, indeed, locations may be unknown. If the locations indeed have a geographical interpretation, NED based theory provides powerful results and a greater ability to handle nonlinear models, see e.g. Xu and Lee (2015). Thus neither approach dominates the other. The results in this paper justify the use of methods developed for fixed $W_{j}$ when the $W_{j}$ are stochastic and, as a result, should provide reassurance to practitioners faced with such $W_{j}$, although the theory requires additional conditions to account for the stochastic nature of the $W_{j}$.

An additional innovation is that we allow for a general 'spatial linear process' structure in the disturbances, cf. Robinson and Thawornkaiwong (2012) and Delgado and Robinson (2015). The former do not consider models with spatial lags in the dependent variables explicitly, nor do they provide theory for OLS or PMLE, while the latter do not consider models with regressors. The spatial weights are deterministic in both papers. In this sense we make a novel contribution to the literature also in the deterministic $W_{j}$ case that we formally cover.

The paper is organized as follows: Section 2 contains asymptotic theory for IV estimates, Section 3 for OLS and Section 4 for PMLE. We discuss implications of common weight matrix normalizations and structures in Section 5, while Section 6 contains a Monte Carlo simulation study that includes two different regimes for generating the spatial weight matrices, with an additional regime discussed in an online appendix associated with this article, available at Cambridge Journals Online (journals.cambridge.org/ect). Section 7 concludes the paper. Two appendices contain theorem proofs and technical lemmas.

\section{IV estimation}

Assume that, for an $n \times 1$ vector of observations $y_{n}$, an $n \times k$ matrix of regressors $X_{n}$ and $n \times n$ weight matrices $W_{j n}, j=1, \ldots, p$, there exist unknown vectors $\lambda=\left(\lambda_{1}, \lambda_{2}, \ldots, \lambda_{p}\right)^{\prime}$ and $\beta \neq 0$ such 
that

$$
y_{n}=\sum_{j=1}^{p} \lambda_{j} W_{j n} y_{n}+X_{n} \beta+u_{n}
$$

where $u_{n}$ is an $n \times 1$ vector of unobserved disturbances. The $W_{j n}$ in (2.1) are sometimes normalized in ways that make their elements dependent on $n$, e.g. row-normalization, and $X_{n}$ may contain spatial lags of basic explanatory variables. Both points imply triangular arrays and justify the $n$ subscripting in (2.1), but the linear process type structure we permit for the disturbances also entails $n$ subscripting on these. Subsequently we will drop $n$ subscripts for brevity, but will occasionally remind the reader of the $n$-dependence of certain quantities.

Let $Z=Z_{n}$ be a matrix of instruments with dimension $n \times p_{1}, p_{1} \geq p$. Denoting $\theta=\left(\lambda^{\prime}, \beta^{\prime}\right)^{\prime}$, define the IV estimate of $\theta$ as

$$
\hat{\theta}=n^{-1} \overline{\bar{Q}}^{-1} \overline{\bar{K}}^{\prime} \bar{J}^{-1}[Z, X]^{\prime} y=\theta+\overline{\bar{Q}}^{-1} \overline{\bar{K}}^{\prime} \bar{J}^{-1} q,
$$

where $\overline{\bar{Q}}=\overline{\bar{Q}}_{n}=\overline{\bar{K}}^{\prime} \bar{J}^{-1} \overline{\bar{K}}($ dimension $p+k)$ and $\overline{\bar{K}}=\overline{\bar{K}}_{n}=n^{-1}[Z, X]^{\prime}[R, X]\left(\operatorname{dimension}\left(p_{1}+k\right) \times\right.$ $(p+k))$, with $R=\left[W_{1} y, \ldots, W_{p} y\right], \bar{J}=\bar{J}_{n}=n^{-1}[Z, X]^{\prime}[Z, X]\left(\right.$ dimension $\left.p_{1}+k\right), q=n^{-1}[Z, X]^{\prime} u$. Throughout the paper $C$ denotes a generic positive constant, arbitrarily large but bounded and independent of $n$.

Assumption 1. (2.1) holds with $u=u_{n}=\left(u_{1 n}, \ldots, u_{n n}\right)^{\prime}$, and

$$
u_{r n}=u_{r}=\sum_{l=1}^{\infty} c_{r l} \epsilon_{l}, \quad r=1, \ldots, n, \quad n \geq 1 \text {, }
$$

where $\epsilon_{l}$ are scalar independent random variables with zero mean and variance $\sigma^{2}, c_{r l}=c_{r l n}$, and satisfy

$$
\sum_{l=1}^{\infty} c_{r l}^{2}<C, \quad r=1, \ldots, n, \quad n \geq 1 .
$$

Assumption 2. The elements of $W_{j}, j=1, \ldots, p$, are random variables that are uniformly $\mathscr{O}_{p}\left(h_{W_{n}}^{-1}\right)$, as $n \rightarrow \infty$, with $h_{W_{n}}=h_{W}$ a bounded or divergent sequence that is bounded away from zero and satisfies $h_{W}=o(n)$ if it is divergent.

Assumption 1 permits a wide variety of disturbance processes including SAR and SMA, and implies that each $u_{r}$ forms a triangular array. The square summability of linear process coefficients in (2.4) allows spatial 'long-memory', while identity of distribution of the $\epsilon_{l}$ is avoided. Robinson and Thawornkaiwong (2012), who introduced this assumption, discuss it in detail. The time series literature commonly allows for martingale $\epsilon_{l}$, but this is avoided in spatial settings as there may be no natural ordering available. Assumption 2 is an extension to stochastic weights of a commonly employed assumption that controls spatial weights, cf. Lee (2002, 2004), Gupta and Robinson (2015). Allowing $h_{W}$ to be bounded can imply a fixed number of neighbours as $n \rightarrow \infty$. Write $S=I_{n}-$ $\sum_{j=1}^{p} \lambda_{j} W_{j}, I_{n}$ denoting the $n$-dimensional identity matrix, and introduce:

Assumption 3. $P(S$ is non-singular $)=1$, for all sufficiently large $n$, at the true parameter value $\lambda=\lambda_{0}$.

For a generic matrix $M$, define $\|M\|$ as the square root of the largest eigenvalue of $M M^{\prime}$ (the spectral norm), $\|M\|_{R}$ as the largest absolute row-sum of $M$ (the maximum row-sum norm) and 
$\|M\|_{F}=\left(\operatorname{tr}\left(M^{\prime} M\right)\right)^{\frac{1}{2}}$ (the Frobenius norm). Assumption 3 ensures that a reduced form exists almost everywhere (a.e.) for $y$, and is satisfied if $P\left(\left\|\sum_{j=1}^{p} \lambda_{j} W_{j}\right\|<1\right)=1$. Indeed, we can write (2.1) as

$$
S y=X \beta+u
$$

or, equivalently, $y=R \lambda+X \beta+u$. Assumption 3 implies that

$$
y=S^{-1} X \beta+S^{-1} u, \text { a.e. }
$$

so $R=A+B$ where $A=\left[G_{1} X \beta, \ldots, G_{p} X \beta\right], B=\left[G_{1} u, \ldots, G_{p} u\right]$ and $G_{j}=W_{j} S^{-1}$ for $j=1, \ldots, p$. Also define $\bar{K}=\bar{K}_{n}=n^{-1}[Z, X]^{\prime}[A, X], \quad \bar{Q}=\bar{Q}_{n}=\bar{K}^{\prime} \bar{J}^{-1} \bar{K}$ and introduce user chosen real numbers $\zeta_{i}, i=1, \ldots, 12$, (because twelve such numbers will be needed in the subsequent results) such that $1<\zeta_{i}<C$ for each $i$ and $\zeta_{j}^{-1}+\zeta_{j+1}^{-1}=1$ for odd $j$. The $\zeta_{i}$ will be used in Hölder inequalities in the proofs.

Assumption 4. $X, W_{j}$ and $z_{r}$ are independent of $\epsilon_{l}, r=1, \ldots, n, l=1, \ldots, j=1, \ldots, p$, where $z_{r}$ is the $r$-th column of $Z^{\prime}$. Let $a_{r j n}=a_{r j}$ denote the $(r, j)$-th element of $[Z, X]$. Then

$$
\max _{1 \leq r \leq n, 1 \leq j \leq p_{1}+k} \mathbb{E}\left|a_{r j}\right|^{2 \zeta_{1}}<C
$$

and, as $n \rightarrow \infty$,

$$
\bar{K} \stackrel{p}{\longrightarrow} K, \bar{J} \stackrel{p}{\longrightarrow} J
$$

where $K$ and $J$ are full-rank constant matrices, with $J$ symmetric.

A consequence of Assumption 4 is that $\bar{Q}-Q=o_{p}(1)$, with $Q=K^{\prime} J^{-1} K$. Condition (2.7) implies finite $2 \zeta_{1}$-th moments for instruments and regressors. The requirement of the whole regressor matrix $X$ being independent of the $\epsilon_{l}$ stems from the fact the instruments are typically constructed using linearly independent columns of $W_{j}^{s} X, j=1, \ldots, p, s \geq 1$, cf. Kelejian and Prucha (1998). Evidently a given instrument vector then contains elements from different rows of $X$, as was noted by Gupta and Robinson (2015).

Denote

$$
\chi_{n}=n^{-\frac{1}{2}} \max _{1 \leq j \leq p}\left(\mathbb{E}\left\|W_{j}\right\|^{2 \zeta_{2} \zeta_{3}}\right)^{\frac{1}{2 \zeta_{2} \zeta_{3}}}\left(\mathbb{E}\left\|S^{-1}\right\|^{2 \zeta_{2} \zeta_{4}}\right)^{\frac{1}{2 \zeta_{2} \zeta_{4}}} .
$$

Theorem 2.1. Let Assumptions 1-4 hold and

$$
\chi_{n} \rightarrow 0 \text {, as } n \rightarrow \infty \text {. }
$$

Then $\hat{\theta}-\theta \stackrel{p}{\longrightarrow} 0$, as $n \rightarrow \infty$.

The condition (2.10) limits the extent of spatial correlation. Note that (2.10) does not impose that $\left\|W_{j}\right\|$ or $\left\|S^{-1}\right\|$ have finite $2 \zeta_{2} \zeta_{3}$-th or $2 \zeta_{2} \zeta_{4}$-th moments, but allows these to grow with $n$. In this sense it is not as strong as may be imagined at first glance. We will look at a specific example with potentially unbounded moments in Section 5. There is an implication of being able to 'trade-off' the magnitude of moments of $\left\|W_{j}\right\|$ and $\left\|S^{-1}\right\|$ in $\chi_{n}$ and $a_{r j}$ by choices of $\zeta_{i}, i=1,2,3,4$. Some of the existing literature on SAR models with fixed weights imposes restrictions on $\left\|W_{j}\right\|_{R}$ or $\left\|S^{-1}\right\|_{R}$, but these are evidently stronger than those based on the spectral norm. Indeed, taking $\zeta_{i}=2$, 
$i=1,2,3,4$, for simplicity, the inequality $\|F\|^{2} \leq\|F\|_{R}\left\|F^{\prime}\right\|_{R}$ immediately implies $\chi_{n}=\mathscr{O}\left(\chi_{n, R}\right)$ with

$$
\chi_{n, R}=n^{-\frac{1}{2}} \max _{1 \leq j \leq p}\left(\mathbb{E}\left\|W_{j}\right\|_{R}^{8} \mathbb{E}\left\|W_{j}^{\prime}\right\|_{R}^{8} \mathbb{E}\left\|S^{-1}\right\|_{R}^{8} \mathbb{E}\left\|S^{\prime-1}\right\|_{R}^{8}\right)^{\frac{1}{16}} .
$$

Let $\mathbb{1}(\cdot)$ denote indicator function.

Assumption 5. $\sup _{l \geq 1} \mathbb{E}\left(\epsilon_{l}^{2} \mathbb{1}\left(\left|\epsilon_{l}\right|>\delta\right)\right) \rightarrow 0$, as $\delta \rightarrow \infty$.

Assumption 6. With $a_{r}^{\prime}$ denoting the $r$-th row of $[Z, X]$ and $\Phi$ a positive definite (p.d.) constant matrix,

$$
\begin{aligned}
& n^{-1} \sum_{r, s=1}^{n} \sum_{l=1}^{\infty} c_{r l} c_{s l} a_{r} a_{s}^{\prime} \stackrel{p}{\longrightarrow} \Phi \text {, as } n \rightarrow \infty, \\
& n^{-1} \sup _{l \geq 1}\left\|\sum_{r=1}^{n} a_{r} c_{r l}\right\|^{2} \stackrel{p}{\longrightarrow} 0, \text { as } n \rightarrow \infty .
\end{aligned}
$$

Assumption 5 avoids identity of distribution for the $\epsilon_{l}$, (2.11) simply asserts convergence of the covariance matrix of $n^{-\frac{1}{2}}[Z, X]^{\prime} u$ while (2.12) is the form of the Lindeberg condition required for the central limit theorem.

Theorem 2.2. Let Assumptions 1-6 and (2.10) hold. Then

$$
n^{\frac{1}{2}}(\hat{\theta}-\theta) \stackrel{d}{\longrightarrow} \mathcal{N}\left(0, \sigma^{2} Q^{-1} K^{\prime} J^{-1} \Phi J^{-1} K Q^{-1}\right) \text {, as } n \rightarrow \infty .
$$

\section{OLS estimation}

Define the OLS estimate

$$
\tilde{\theta}=n^{-1} \overline{\bar{L}}^{-1}[R, X]^{\prime} y=\theta+\overline{\bar{L}}^{-1} w
$$

where $\overline{\bar{L}}=\overline{\bar{L}}_{n}=n^{-1}[R, X]^{\prime}[R, X]$ (dimension $\left.p+k\right), \ell=\ell_{n}=n^{-1}[R, X]^{\prime} u$. Also define $\bar{L}=\bar{L}_{n}=$ $n^{-1}[A, X]^{\prime}[A, X]$. Assumption 2 needs to be strengthened to the following sufficient condition:

Assumption 7. The $\zeta_{i}$ are chosen such that $\zeta_{5} \zeta_{7}=2 \zeta_{11}$ and

$$
\max _{1 \leq j \leq p} \mathbb{E}\left(\left\{\max _{1 \leq r, s \leq n}\left|w_{r s, j}\right|\right\}^{2 \zeta_{11}}\right)=\mathscr{O}\left(h_{W}^{-2 \zeta_{11}}\right),
$$

where $w_{r s, j}$ is the $(r, s)$-th element of $W_{j}, j=1, \ldots, p$.

This assumption implies $\max _{1 \leq r, s \leq n, 1 \leq j \leq p}\left|w_{r s, j}\right|=\mathscr{O}_{p}\left(h_{W}^{-1}\right)$. Various bounds depending on the distribution of $w_{r s, j}$ exist in the extreme value literature for the expectation, but Assumption 7 ensures also that the familiar case with fixed $w_{r s, j}=\mathscr{O}\left(h_{W}^{-1}\right)$, uniformly in $r, s$, is formally covered. The restriction $\zeta_{5} \zeta_{7}=2 \zeta_{11}$ is satisfied in the case where the Cauchy Schwarz inequality is used in place of the Hölder inequality, implying that $\zeta_{i}=2$ for all $i$.

Assumption 8. $X$ and $W_{j}$ are independent of $\epsilon_{l}, l=1, \ldots, j=1, \ldots, p$. Let $t_{r j n}=t_{r j}$ denote the $(r, j)$-th element of $[A, X]$. Then

$$
\max _{1 \leq r \leq n, 1 \leq j \leq p+k} \mathbb{E}\left|t_{r j}\right|^{2 \zeta_{1}}<C
$$


and, as $n \rightarrow \infty$,

$$
\bar{L} \stackrel{p}{\longrightarrow} L,
$$

where $L$ is a constant, symmetric and non-singular matrix.

Define

$$
\pi_{n}=h_{W}^{-\frac{1}{2}}\left(\max _{1 \leq j \leq p} \mathbb{E}\left\|W_{j}^{\prime}\right\|_{R}^{\zeta_{6} \zeta_{9}}\right)^{\frac{1}{2 \zeta_{6} \zeta_{9}}}\left(\mathbb{E}\left\|S^{\prime-1}\right\|_{R}^{\zeta_{5} \zeta_{8}}\right)^{\frac{1}{2 \zeta_{5} \zeta_{8}}}\left(\mathbb{E}\left\|S^{\prime-1}\right\|_{R}^{\zeta_{6} \zeta_{10}}\right)^{\frac{1}{2 \zeta_{6} \zeta_{10}}} .
$$

Theorem 3.1. Let Assumptions 1-3, 7, 8 hold and

$$
h_{W}^{-1}+\chi_{n}+\pi_{n} \longrightarrow 0 \text { as } n \rightarrow \infty
$$

Then $\tilde{\theta}-\theta \stackrel{p}{\longrightarrow} 0$, as $n \rightarrow \infty$.

For consistency of OLS estimates $h_{W} \rightarrow \infty$ is necessary even with deterministic $W_{j}$ (cf. Lee (2002), Gupta and Robinson (2015)), and (3.5) strengthens the restrictions on spatial correlation relative to $h_{W}$. Our conditions match those in the aforementioned literature except the additional requirements on moments of $\left\|W_{j}\right\|$ and $\left\|S^{-1}\right\|$ which arise because these are stochastic.

Taking $p=1$ for simplicity (and writing $W_{1}=W$ ), an example of non-stochastic $W$ for which $h_{W} \rightarrow \infty$ can be constructed as in Case (1991), where each of $d$ districts contains $m$ farmers, so $n=m d$. Interdistrict independence is assumed, as is equal reaction within districts, implying

$$
W=I_{d} \otimes B_{m}, \text { with } B_{m}=(m-1)^{-1}\left(l_{m} l_{m}^{\prime}-I_{m}\right),
$$

where $l_{m}$ is the $m$-dimensional vector of ones and $\otimes$ denotes Kronecker product. Here $h_{W}=m-1$, which diverges as $m \rightarrow \infty$, and thus satisfies (3.5). More general versions of such specifications are also studied recently in Hillier and Martellosio (2018).

The specification given in (3.6) consists of a block-diagonal $W$ with very dense blocks. On the other hand, specifications of $W$ can be of the more sparse variety, in which case $h_{W}$ will be bounded and (3.5) will fail. As an example consider the case of circulant $W$, where each unit has only one neighbour on 'either side'. Mathematically, define $W^{*}$ as the symmetric circulant matrix with first row elements given by

$$
w_{1 r}^{*}= \begin{cases}0 & \text { if } r=1 \text { or } r=3, \ldots, n-1 \\ \omega & \text { if } r=2 \text { or } r=n,\end{cases}
$$

with $\omega$ some known real number, see e.g. Das, Kelejian, and Prucha (2003). A binary neighbourhood criterion would take $\omega=1$. Now define

$$
W=W^{*} /\left\|W^{*}\right\|
$$

where $\left\|W^{*}\right\|=2 \omega$, because $W^{*}$ is a symmetric, circulant matrix (see e.g. Davis (1979) p. 73). Then $W$ is also a symmetric circulant matrix with first row elements given by $w_{1 r}^{*} / 2 \omega$. In this case $h_{W}=2 \omega$, which does not diverge with $n$ in general.

Assumption 9. $\sup _{l \geq 1} \mathbb{E}\left(\epsilon_{l}^{4} \mathbb{1}\left(\left|\epsilon_{l}\right|>\delta\right)\right) \rightarrow 0$, as $\delta \rightarrow \infty$.

This assumption seems hard to relax for a CLT. Indeed, even for (2.1) with $p=1, W_{j}$ fixed and no 
linear process structure, Lee (2002) required $\mathbb{E}\left|u_{r}\right|^{4+\eta}<C$, for some $\eta>0$. Gupta and Robinson (2015) relaxed this slightly to $\mathbb{E} u_{r}^{4}<C$, with increasing $p, k$ but restricted themselves to iid $u_{r}$. Here we avoid identity of distribution of $\epsilon_{l}$, and $u_{r}$, but require the uniform integrability of the $\epsilon_{l}^{4}$ that Assumption 9 entails.

Assumption 10. With $t_{r}^{\prime}$ denoting the r-th row of $[A, X]$ and $\Psi$ a p.d. constant matrix,

$$
\begin{gathered}
n^{-1} \sum_{r, s=1}^{n} \sum_{l=1}^{\infty} c_{r l} c_{s l} t_{r} t_{s}^{\prime} \quad \stackrel{p}{\longrightarrow} \Psi, \text { as } n \rightarrow \infty, \\
n^{-1} \sup _{l \geq 1}\left\|\sum_{r=1}^{n} t_{r} c_{r l}\right\|^{2} \stackrel{p}{\longrightarrow} \quad 0, \text { as } n \rightarrow \infty .
\end{gathered}
$$

Theorem 3.2. Let Assumptions 1-3, 7-9 and (3.5) hold and

$$
h_{W}^{-1} n^{\frac{1}{2}}\left(\mathbb{E}\left\|S^{\prime-1}\right\|_{R}^{2 \zeta_{12}}\right)^{\frac{1}{2 \zeta_{12}}} \longrightarrow 0, \text { as } n \rightarrow \infty .
$$

Then

$$
n^{\frac{1}{2}}(\tilde{\theta}-\theta) \stackrel{d}{\longrightarrow} \mathcal{N}\left(0, \sigma^{2} L^{-1} \Psi L^{-1}\right) \text {, as } n \rightarrow \infty .
$$

The proof requires some care to ensure that (2.4) does not need strengthening. Lee (2002) established that asymptotic normality of OLS relies not just on divergence of $h_{W}$, but sufficiently fast divergence, viz. $n^{\frac{1}{2}}=o\left(h_{W}\right)$. Condition (3.11) indicates the additional requirement that arises when the $W_{j}$ are stochastic and reduces to the condition in Lee (2002) for non-stochastic $W_{j}$. In the $W$ described in (3.6) we have $h_{W}=m-1$ and $n=m d$, so (3.11) is satisfied if $d^{\frac{1}{2}} / m^{\frac{1}{2}} \rightarrow 0$ as $d, m \rightarrow \infty$. On the other hand, the $W$ of (3.8) will not satisfy (3.11) in general.

With all $\zeta_{i}=2$, we obtain

$$
\begin{aligned}
\chi_{n} & =n^{-\frac{1}{2}}\left(\max _{1 \leq j \leq p} \mathbb{E}\left\|W_{j}\right\|^{8} \mathbb{E}\left\|S^{-1}\right\|^{8}\right)^{\frac{1}{8}} \\
\pi_{n} & =h_{W}^{-\frac{1}{2}}\left(\max _{1 \leq j \leq p} \mathbb{E}\left\|W_{j}^{\prime}\right\|_{R}^{4}\right)^{\frac{1}{8}}\left(\mathbb{E}\left\|S^{\prime-1}\right\|_{R}^{4}\right)^{\frac{1}{4}}
\end{aligned}
$$

$(3.11)$ becomes $h_{W}^{-1} n^{\frac{1}{2}}\left(\mathbb{E}\left\|S^{\prime-1}\right\|_{R}^{4}\right)^{\frac{1}{4}} \rightarrow 0$, as $n \rightarrow \infty$, and $(2.7),(3.2)$ require finite fourth moments for the $a_{r j}$ and $t_{r j}$ respectively.

In both Theorems 2.2 and 3.2, if the $W_{j}$ are taken to be deterministic and the $u_{r}$ are iid, the limit distributions are identical to those obtained by Gupta and Robinson (2015), taking $p$ and $k$ to be fixed in their results.

\section{Pseudo maximum likelihood estimation}

In this section, we parameterize the coefficients in the linear process given in Assumption 1 . In particular, suppose there is an unknown $q \times 1$ vector $\tau_{0} \in \mathcal{T}$ such that $c_{r l}=c_{r l}\left(\tau_{0}\right)$, all $r=1, \ldots, n$ and $l \geq 1$. Writing $\sigma_{0}^{-2} \mathbb{E}\left(u_{n} u_{n}^{\prime}\right)=\Xi_{n}\left(\tau_{0}\right) \equiv \Xi\left(\tau_{0}\right)$, the pseudo likelihood function based on Gaussianity 
and conditional on $W_{j}, j=1, \ldots, p, X$, is

$$
\log \left(2 \pi \sigma^{2}\right)-\frac{2}{n} \log |S(\lambda)|+\frac{1}{n} \log |\Xi(\tau)|+\frac{1}{\sigma^{2} n}(S(\lambda) y-X \beta)^{\prime} \Xi(\tau)^{-1}(S(\lambda) y-X \beta),
$$

at any admissible point $\left(\varrho^{\prime}, \sigma^{2}\right)^{\prime}$ with $\varrho=\left(\theta^{\prime}, \tau^{\prime}\right)^{\prime}$, for nonsingular $S(\lambda)$ and $\Xi(\tau)$, although Gaussianity is nowhere assumed. True parameter values are identified by a zero subscript and for any parameter $\alpha$ and any quantity $x(\alpha)$ we adopt the convention $x\left(\alpha_{0}\right) \equiv x$.

For given $\gamma=\left(\lambda^{\prime}, \tau^{\prime}\right)^{\prime},(4.1)$ is minimised with respect to $\beta$ and $\sigma^{2}$ by

$$
\begin{aligned}
\bar{\beta}(\gamma) & =\left(X^{\prime} \Xi(\tau)^{-1} X\right)^{-1} X^{\prime} \Xi(\tau)^{-1} S(\lambda) y, \\
\bar{\sigma}^{2}(\gamma) & =n^{-1} y^{\prime} S^{\prime}(\lambda) \Xi(\tau)^{\prime-\frac{1}{2}} M(\tau) \Xi(\tau)^{-\frac{1}{2}} S(\lambda) y,
\end{aligned}
$$

where $M(\tau)=I_{n}-\Xi(\tau)^{-\frac{1}{2}} X\left(X^{\prime} \Xi(\tau)^{\prime-\frac{1}{2}} \Xi(\tau)^{-\frac{1}{2}} X\right)^{-1} X^{\prime} \Xi(\tau)^{\prime-\frac{1}{2}}$ and $\Xi(\tau)^{-\frac{1}{2}} \equiv \Xi_{n}(\tau)^{-\frac{1}{2}}$ is the $n \times n$ matrix such that $\Xi(\tau)^{\prime-\frac{1}{2}} \Xi(\tau)^{-\frac{1}{2}}=\Xi(\tau)^{-1}$. This is a slight abuse of notation but makes the proofs easier to read. The PMLE of $\gamma_{0}$ is $\check{\gamma}=\arg \min _{\gamma \in \Gamma} \mathcal{L}(\gamma)$, where

$$
\mathcal{L}(\gamma)=\log \bar{\sigma}^{2}(\gamma)+n^{-1} \log \left|S^{\prime-1}(\lambda) \Xi(\tau) S^{-1}(\lambda)\right|,
$$

and $\Gamma=\Lambda \times \mathcal{T}$ is taken to be a compact subset of $\mathbb{R}^{p+q}$. The PMLEs of $\beta_{0}$ and $\sigma_{0}^{2}$ are defined as $\bar{\beta}(\check{\gamma}) \equiv \check{\beta}$ and $\bar{\sigma}^{2}(\check{\gamma}) \equiv \check{\sigma}^{2}$ respectively.

Assumption 11. For all sufficiently large $n$, the $u_{r}$ have uniformly bounded fourth moment, $r=$ $1, \ldots, n$.

Assumption 12. $\max _{j=1, \ldots, p}\left\|W_{j}\right\|+\left\|S^{-1}\right\|=\mathscr{O}_{p}(1)$.

Assumption 13. $\varlimsup_{n \rightarrow \infty} \sup _{\tau \in \mathcal{T}}\left(\|\Xi(\tau)\|+\left\|\Xi(\tau)^{-1}\right\|\right)<\infty$.

Assumption 14. For any $\tau^{\dagger} \in \mathcal{T}$ and any $\eta>0$, there exists $\varepsilon>0$ such that

$$
\varlimsup_{n \rightarrow \infty} \sup _{\tau \in\left\{\tau:\left\|\tau-\tau^{\dagger}\right\|<\varepsilon\right\} \cap \mathcal{T}}\left\|\Xi(\tau)-\Xi\left(\tau^{\dagger}\right)\right\|<\eta
$$

Assumption 12 implies that $\max _{j=1, \ldots, p}\left\|G_{j}\right\|=\mathscr{O}_{p}(1)$. The approach to conditions on the $W_{j}$ and $S^{-1}$ differ somewhat in this section as compared to the previous two. There we focused on more primitive conditions based on moments of $\left\|W_{j}\right\|$ and $\left\|S^{-1}\right\|$, and also allowed a great amount of flexibility both in the way the Hölder inequality is applied (exemplified by the twelve different $\zeta_{i}$ ) and the fact that the moments may diverge at a certain rate. The PMLE considered in this section is inherently harder to derive asymptotic properties for because it is only implicitly defined, and matters are complicated further also by the presence of $\Xi(\tau)$ in the objective function (4.1). Thus to maintain tractability we prefer conditions of the type given in Assumption 12. Assumption 13 is a standard type of regularity condition that may be regarded as guaranteeing asymptotic boundedness and non-singularity. Assumption 14 also holds with $\Xi(\tau)-\Xi\left(\tau^{\dagger}\right)$ replaced by $\Xi(\tau)^{-1}-\Xi\left(\tau^{\dagger}\right)^{-1}$ due to Assumption 13, and is useful in an equicontinuity argument in the consistency proof. Further discussion of it may be found in Delgado and Robinson (2015), who also discuss sufficient conditions for it to hold. Write $T(\lambda)=S(\lambda) S^{-1}$ and define $\sigma^{2}(\gamma)=n^{-1} \sigma_{0}^{2} \operatorname{tr}\left(T^{\prime}(\lambda) \Xi(\tau)^{-1} T(\lambda) \Xi\right)=n^{-1} \sigma_{0}^{2}\left\|\Xi(\tau)^{-\frac{1}{2}} T(\lambda) \Xi^{\frac{1}{2}}\right\|_{F}^{2}$, which is nonnegative by definition and bounded in probability by Assumptions 12 and 13 . 
Assumption 15. There exist positive and finite constants $c$ and $C$ such that $c \leq \sigma^{2}(\gamma) \leq C$ with probability one, for all $\gamma \in \Gamma$.

Assumption 16. $\gamma_{0} \in \Gamma$ and, for any $\eta>0$,

$$
P\left(\varliminf_{n \rightarrow \infty} \inf _{\gamma \in \overline{\mathcal{N}}^{\gamma}(\eta)} \frac{n^{-1} \operatorname{tr}\left(T^{\prime}(\lambda) \Xi(\tau)^{-1} T(\lambda) \Xi\right)}{\left|T^{\prime}(\lambda) \Xi(\tau)^{-1} T(\lambda) \Xi\right|^{1 / n}}>1\right)=1
$$

where $\overline{\mathcal{N}}^{\gamma}(\eta)=\Gamma \backslash \mathcal{N}^{\gamma}(\eta)$ and $\mathcal{N}^{\gamma}(\eta)=\left\{\gamma:\left\|\gamma-\gamma_{0}\right\|<\eta\right\} \cap \Gamma$.

The trace in the numerator inside the probability in (4.6) equals $\left\|\Xi(\tau)^{-\frac{1}{2}} T(\lambda) \Xi^{\frac{1}{2}}\right\|_{F}^{2}$, while the determinant in the denominator is similarly $\left|\Xi(\tau)^{-\frac{1}{2}} T(\lambda) \Xi^{\frac{1}{2}}\right|^{2}$. Thus the inequality between arithmetic and geometric means implies that the ratio inside the probability is at least one. On the other hand, when $\gamma=\gamma_{0}$ the ratio equals one, so (4.6) essentially requires that this situation not arise outside an arbitrarily small neighbourhood of $\gamma_{0}$. Therefore it is an identification condition which can be compared to those already in the literature. The closest conditions may be found in Gupta and Robinson (2018) and Delgado and Robinson (2015), the latter showing the equivalence of their condition to that of Lee (2004) in the case of SAR models. With deterministic weight matrices, the latter interprets the condition as related to the uniqueness of the covariance matrix of $y$. Our setup is rather different, with a SAR model coupled with linear process disturbances and stochastic weight matrices, for which our condition accounts. A Lee (2004)-like interpretation is possible in terms of the uniqueness of the covariance matrix of $y$ conditional on the $W_{j}$ and $X, j=1, \ldots, p$, viz. $S^{-1} \Xi S^{\prime-1}$, because the logarithm of the ratio inside the probability equals $n^{-1} \log \left|\sigma^{2}(\gamma) S(\lambda)^{-1} \Xi(\tau) S^{\prime}(\lambda)^{-1}\right|-n^{-1} \log \left|\sigma_{0}^{2} S^{-1} \Xi S^{\prime-1}\right|$, cf. also equation (37) and the discussion thereafter in Delgado and Robinson (2015).

Assumption 17. $\beta_{0} \neq 0$ and, for any $\eta>0$,

$$
P\left(\lim _{n \rightarrow \infty} \inf _{\left(\lambda^{\prime}, \tau^{\prime}\right)^{\prime} \in \Lambda \times \overline{\mathcal{N}}^{\tau}(\eta)} n^{-1} \beta_{0}^{\prime} X^{\prime} T^{\prime}(\lambda) \Xi(\tau)^{\prime-\frac{1}{2}} M(\tau) \Xi(\tau)^{-\frac{1}{2}} T(\lambda) X \beta_{0} /\left\|\beta_{0}\right\|^{2}>0\right)=1 .
$$

Because the event inside the probability is equivalent (by straightforward minimization) to

$$
\varliminf_{n \rightarrow \infty} \min _{\beta \in \mathbb{R}^{k}} \inf _{\left(\lambda^{\prime}, \tau^{\prime}\right)^{\prime} \in \Lambda \times \overline{\mathcal{N}}^{\tau}(\eta)} n^{-1}\left(X \beta-T(\lambda) X \beta_{0}\right)^{\prime} \Xi(\tau)^{-1}\left(X \beta-T(\lambda) X \beta_{0}\right) /\left\|\beta_{0}\right\|^{2}>0
$$

Assumption 17 is in fact an identification condition similar to one used for nonlinear regression in a SAR model by Gupta and Robinson (2018). Let $\bar{\varphi}(A)$ (respectively $\underline{\varphi}(A)$ ) denote the largest (respectively smallest) eigenvalue of a generic square nonnegative definite matrix $A$. Then a necessary condition for the event in (4.7) is that

$$
\varliminf_{n \rightarrow \infty} \inf _{\left(\lambda^{\prime}, \tau^{\prime}\right)^{\prime} \in \Lambda \times \overline{\mathcal{N}}^{\tau}(\eta)} \underline{\varphi}\left(n^{-1} X^{\prime} T^{\prime}(\lambda) \Xi(\tau)^{\prime-\frac{1}{2}} M(\tau) \Xi(\tau)^{-\frac{1}{2}} T(\lambda) X\right)>0 .
$$

The final identification condition is a familiar asymptotic full-rank condition

Assumption 18. $\left\{\underline{\varphi}\left(n^{-1} X^{\prime} X\right)\right\}^{-1}=\mathscr{O}_{p}(1)$.

This assumption implies $\left\{\sup _{\tau \in \mathcal{T}} \underline{\varphi}\left(n^{-1} X^{\prime} \Xi(\tau)^{-1} X\right)\right\}^{-1}=\mathscr{O}_{p}(1)$ in view of Assumption 13. 
Theorem 4.1. Let Assumptions 1-3, 8 and 11-17 hold. Then $\left(\varrho^{\prime}, \check{\sigma}^{2}\right)^{\prime}-\left(\varrho_{0}^{\prime}, \sigma_{0}^{2}\right)^{\prime} \stackrel{p}{\longrightarrow} 0$ as $n \rightarrow \infty$.

Assumption 19. $\gamma_{0}$ is an interior point of $\Gamma$.

Assumption 20. The $\epsilon_{j}$ in Assumption 1 have finite third and fourth moments $\mu_{3}$ and $\mu_{4}$ respectively, finite eighth moment and, denoting by $\xi_{r s}(\tau)$ the $(r, s)$-th element of $\Xi(\tau)$ and defining

$$
c_{r j}^{*}=c_{r j} / \xi_{r r}^{\frac{1}{2}}, r=1, \ldots, n, n \geq 1, l \geq 1
$$

we have

$$
\varlimsup_{n \rightarrow \infty} \sup _{r=1, \ldots, n} \sum_{l=1}^{\infty}\left|c_{r l}^{*}\right|+\sup _{l \geq 1} \varlimsup_{n \rightarrow \infty} \sum_{r=1}^{n}\left|c_{r l}^{*}\right|<\infty .
$$

Assumption 19 is standard for a central limit theorem for implicitly defined estimates. Assumption 20 is introduced by Delgado and Robinson (2015), and is discussed therein. It implies that Assumption 11 holds.

Assumption 21. $\max _{j=1, \ldots, p}\left\|W_{j}\right\|_{R}+\max _{j=1, \ldots, p}\left\|W_{j}^{\prime}\right\|_{R}+\left\|S^{-1}\right\|_{R}+\left\|S^{\prime-1}\right\|_{R}+\|X\|_{R}=\mathscr{O}_{p}(1)$ as $n \rightarrow \infty$.

Assumption 21 is quite standard even in the literature with non-stochastic $W_{j}$ and $X$, where the $\mathscr{O}_{p}(1)$ requirement is replaced by simply $\mathscr{O}(1)$. Note that $\|X\|_{R}=\mathscr{O}(1)$ would then be satisfied if $X$ has uniformly bounded, constant elements. Let $\Xi_{i_{1}}(\tau)=\partial \Xi(\tau) / \partial \tau_{i_{1}}, \Xi_{i_{1} i_{2}}(\tau)=\partial \Xi_{i_{1}}(\tau) / \partial \tau_{i_{2}}$ and $\Xi_{i_{1} i_{2} i_{3}}(\tau)=\partial \Xi_{i_{1} i_{2}}(\tau) / \partial \tau_{i_{3}}, i_{1}, i_{2}, i_{3}=1, \ldots, q$, where the matrices are differentiated element-wise and the existence of the derivatives is guaranteed by Assumption 22 below, with typical elements $\xi_{r s i_{1}}, \xi_{r s i_{1} i_{2}}$ and $\xi_{r s i_{1} i_{2} i_{3}}$ respectively.

Assumption 22. For $i_{1}, i_{2}, i_{3}=1, \ldots, q$ and all sufficiently large $n$, the elements of $\Xi$ are thrice continuously differentiable, for any $\eta>0$

$$
\varlimsup_{n \rightarrow \infty} \sup _{\tau \in \mathcal{N}^{\tau}(\eta)}\left(\left\|\Xi(\tau)^{-1}\right\|_{R}+\left\|\Xi_{i_{1}}(\tau)\right\|_{R}+\left\|\Xi_{i_{1} i_{2}}(\tau)\right\|_{R}+\left\|\Xi_{i_{1} i_{2} i_{3}}(\tau)\right\|_{R}\right)<\infty
$$

and for a positive sequence $h_{\Xi} \equiv h_{\Xi n}$ that is either bounded or divergent and satisfies $h_{\Xi} / n \rightarrow 0$ as $n \rightarrow \infty$, we have

$$
\varlimsup_{n \rightarrow \infty} \sup _{\tau \in \mathcal{N}^{\tau}(\eta)} \sup _{r, s=1, \ldots, n} h_{\Xi}\left(\left|\xi_{r s}(\tau)\right|+\left|\xi_{r s i_{1}}(\tau)\right|+\left|\xi_{r s i_{1} i_{2}}(\tau)\right|+\left|\xi_{r s i_{1} i_{2} i_{3}}(\tau)\right|\right)<\infty
$$

Assumptions 21 and 22 serve to control the extent of spatial correlation to a greater degree than Assumptions 12 and 13 in view of the inequalities $\|A\|^{2} \leq\|A\|_{R}\left\|A^{\prime}\right\|_{R}$ and $\|A\| \leq\|A\|_{F}$ for a generic matrix $A$. Assumption 22 also imposes local smoothness conditions on $\Xi(\tau)$.

The second derivative matrix of $(4.1)$ at $\left(\varrho, \sigma^{2}\right)$ is denoted $H\left(\varrho, \sigma^{2}\right)$. Let $P_{G 1}(\lambda)$ and $P_{G 2}(\lambda)$ denote the $p \times p$ matrices with $(i, j)$-th elements $\operatorname{tr}\left(G_{j}(\lambda) G_{i}(\lambda)\right), \operatorname{tr}\left(G_{j}^{\prime}(\lambda) G_{i}(\lambda)\right)$, respectively, $P_{\Xi}(\tau)$ the $q \times q$ matrix with $(i, j)$-th element $\operatorname{tr}\left(\Xi(\tau)^{-1} \Xi_{i}(\tau) \Xi(\tau)^{-1} \Xi_{j}(\tau)\right)$ and $\Upsilon(\tau)$ the $q \times q$ matrix with $(i, j)$-th element $\operatorname{tr}\left(G_{i} \Xi(\tau)^{-1} \Xi_{j}(\tau)\right)$. Then

$$
\Sigma=\mathbb{E}(H)=2 \sigma_{0}^{-2} n^{-1}\left[\begin{array}{ccc}
\sigma_{0}^{2}\left(P_{G 1}+P_{G 2}\right)+A^{\prime} \Xi^{-1} A & A^{\prime} \Xi^{-1} X & \sigma_{0}^{2} \Upsilon \\
* & X^{\prime} \Xi^{-1} X & 0 \\
* & * & 2^{-1} \sigma_{0}^{2} P_{\Xi}
\end{array}\right]
$$


where the expectation is taken conditional on $W_{j}$ and $X, j=1, \ldots, p$. We also need the conditional covariance matrix of the first derivative of (4.1) at the true parameter values, which we write as $n^{-1}(2 \Sigma+\Omega)$. The next paragraph is devoted to defining $\Omega$, for which purpose let $c_{l}(\tau)$ be the $n \times 1$ vector with $r$-th element $c_{r l}(\tau)$.

Introduce the following matrices: the $n \times p$ and $p \times p$ (respectively) matrices $F_{1}$ and $F_{2}$ with $j$-th column $\sum_{l=1}^{\infty} \Xi^{-1} c_{l} c_{l}^{\prime} G_{j} \Xi^{-1} c_{l}$ and $(i, j)$-th element $\sum_{l=1}^{\infty} c_{l}^{\prime} G_{i} \Xi^{-1} c_{l} c_{l}^{\prime} G_{j} \Xi^{-1} c_{l}$, respectively, the $n \times p$ matrix $F_{3}$ with $j$-th column $\sum_{l=1}^{\infty} \Xi^{-1} c_{l} c_{l}^{\prime} \Xi^{-1} G_{j}^{\prime} c_{l}$, the $n \times q$ and $p \times q$ (respectively) matrices $F_{4}$ and $F_{5}$ with $j$-th column $\sum_{l=1}^{\infty} \Xi^{-1} c_{l} c_{l}^{\prime} \Xi^{-1} \Xi_{j} \Xi^{-1} c_{l}$ and $(i, j)$-th element $\sum_{l=1}^{\infty} c_{l}^{\prime} G_{i} \Xi^{-1} c_{l} c_{l}^{\prime} \Xi^{-1} \Xi_{j} \Xi^{-1} c_{l}$, respectively, the $n \times q$ matrix $F_{6}$ with $j$-th column $\sum_{l=1}^{\infty} \Xi^{-1} c_{l} c_{l}^{\prime} \Xi^{-1} \Xi_{i} \Xi^{-1} c_{l}$ and the $q \times q$ matrix $F_{7}$ with $(i, j)$-th element given by $\sum_{l=1}^{\infty} c_{l}^{\prime} \Xi^{-1} \Xi_{i} \Xi^{-1} c_{l} c_{l}^{\prime} \Xi^{-1} \Xi_{j} \Xi^{-1} c_{l}$. Then

$$
\Omega=\sigma_{0}^{-4} n^{-1}\left[\begin{array}{ccc}
4 \mu_{3}\left(A^{\prime} F_{1}+F_{1}^{\prime} A\right)+4\left(\mu_{4}-3 \sigma_{0}^{4}\right) F_{2} & 4 \mu_{3} F_{3}^{\prime} X & 2 \mu_{3} A^{\prime} F_{4}+2\left(\mu_{4}-3 \sigma_{0}^{4}\right) F_{5} \\
* & 0 & 2 \mu_{3} X^{\prime} F_{6} \\
* & * & \left(\mu_{4}-3 \sigma_{0}^{4}\right) F_{7}
\end{array}\right] .
$$

Let $N=\operatorname{diag}\left[I_{p}, I_{k}, I_{q} h_{\Xi}^{\frac{1}{2}}\right]$.

Assumption 23. The matrices $\Delta=\operatorname{plim}_{n \rightarrow \infty} N(2 \Sigma+\Omega) N$ and $\Pi=\operatorname{plim}_{n \rightarrow \infty} N \Sigma N$ exist and are positive definite.

Theorem 4.2. Let Assumptions 1-3, 8 and 12-23 hold. Then

$$
n^{\frac{1}{2}} N^{-1}\left(\varrho \varrho \varrho_{0}\right) \stackrel{d}{\rightarrow} \mathcal{N}\left(0, \Pi^{-1} \Delta \Pi^{-1}\right), \text { as } n \rightarrow \infty .
$$

The differential norming entailed by $N^{-1}$ is due to the fact that the elements of $P_{\Xi}$ are $\mathscr{O}\left(h_{\Xi}^{-1}\right)$ and will thus all converge to zero when $h_{\Xi}$ is divergent, causing singularity of the limiting covariance matrix. The implication is that when $h_{\Xi}$ diverges $\check{\tau}$ is $n^{\frac{1}{2}} / h_{\Xi}^{\frac{1}{2}}$-consistent, whereas $n^{\frac{1}{2}}$-consistency is achieved when $h_{\Xi}$ is bounded.

Some simplifications are possible in $\Pi$ and $\Delta$ when $h_{W}$ is divergent. The reason is as follows: asymptotic normality of $\varrho \varrho-\varrho_{0}$ is established via the asymptotic normality of the first derivative of (4.1) with respect to $\varrho$. This derivative, given by (A.36) in Appendix A, involves the quadratic in $u$ terms $n^{-1} u^{\prime} G_{j} \Xi^{-1} u-n^{-1} \sigma_{0}^{2} t r G_{j}, j=1, \ldots, p$, which are readily shown to be negligible when $h_{W}$ diverges. We omit the proof of this negligibility to conserve space, and also because it is straightforward given the techniques used in the proof of Theorem 4.2. Thus these quadratic entries can be ignored while establishing asymptotic normality and do not contribute to the asymptotic covariance matrix, eliminating $P_{G_{2}}$ in $\Sigma$ and all blocks of $\Omega$ involving $F_{1}, F_{2}$ and $F_{3}$. Also, because $n^{-1} P_{G_{1}}$ has elements that are uniformly $\mathscr{O}_{p}\left(h_{W}^{-1}\right)$ by Lemma B.6, this part of $\Sigma$ is negligible if $h_{W}$ diverges. In sum, we can replace $\Sigma$ and $\Omega$ by

$$
2 \sigma_{0}^{-2} n^{-1}\left[\begin{array}{ccc}
A^{\prime} \Xi^{-1} A & A^{\prime} \Xi^{-1} X & \sigma_{0}^{2} \Upsilon \\
* & X^{\prime} \Xi^{-1} X & 0 \\
* & * & 2^{-1} \sigma_{0}^{2} P_{\Xi}
\end{array}\right]
$$


and

$$
\sigma_{0}^{-4} n^{-1}\left[\begin{array}{ccc}
0 & 0 & 2 \mu_{3} A^{\prime} F_{4}+2\left(\mu_{4}-3 \sigma_{0}^{4}\right) F_{5} \\
* & 0 & 2 \mu_{3} X^{\prime} F_{6} \\
* & * & \left(\mu_{4}-3 \sigma_{0}^{4}\right) F_{7}
\end{array}\right]
$$

respectively.

Furthermore, suppose that $p=1$, the $u_{i}$ in (2.1) are uncorrelated and identically distributed normal random variables. Thus there is no parameterization by $\tau, \Omega=0$ and we can replace $\Sigma$ by

$$
2 \sigma_{0}^{-2} n^{-1}\left[\begin{array}{cc}
\left(G X \beta_{0}\right)^{\prime}\left(G X \beta_{0}\right) & \left(G X \beta_{0}\right)^{\prime} X \\
* & X^{\prime} X
\end{array}\right],
$$

which matches the distribution obtained in this setting by Lee (2004) in his Theorem 3.2.

\section{Normalizations of weight matrices}

In this section we discuss the effect of various normalizations of the $W_{j}$ on (2.10) and (3.5). For simplicity of exposition we will focus on the case when $\zeta_{i}=2$, all $i$, given in (3.12) and (3.13). Due to the $n^{-\frac{1}{2}}$ factor it is not necessary that the elements of $W_{j}$ and $S^{-1}$ have finite eighth moments for (2.10) to hold, but for given $n$ it is not sufficient either. Similarly due to the $h_{W}^{-\frac{1}{2}}$ factor and $h_{W} \rightarrow \infty$ finite fourth moments for elements of $W_{j}^{\prime}$ or $S^{\prime-1}$ are neither necessary nor sufficient, for given $n$, for (3.5) to hold. Both (2.10) and (3.5) can be compared to conditions imposed in Gupta and Robinson (2015) for deterministic $W_{j}$ elements, where $\max _{1 \leq j \leq p}\left\|W_{j}\right\|+\left\|S^{-1}\right\| \leq C$ was assumed, for which a necessary condition was boundedness of the elements of $W_{j}, S^{-1}$. Thus (2.10) and (3.5) may be viewed as controlling the spatial correlation asymptotically, and in particular controlling the magnitudes of the moments of the $W_{j}$ and $S^{-1}$.

Various sufficient conditions may be found for (2.10) and (3.5) to hold. For example, suppose that

$$
W_{j}=W_{j}^{*} /\left\|W_{j}^{*}\right\|,
$$

for some matrices $W_{j}^{*}$. Then $\left\|W_{j}\right\|^{s} \leq 1$, for any $s>0$, while

$$
\left\|S^{-1}\right\| \leq \sum_{l=0}^{\infty}\left(\sum_{j=1}^{p}\left\|\lambda_{j} W_{j}\right\|\right)^{l} \leq C,
$$

if

$$
\sum_{j=1}^{p}\left|\lambda_{j}\right|<1
$$

The $W_{j}$ can have a special 'single nonzero diagonal block' structure in some applications. Here there are $m_{j} \times m_{j}$ matrices $V_{j}, j=1, \ldots, p$ and $\sum_{j=1}^{p} m_{j}=n$, such that each $W_{j}$ has $V_{j}$ as its $j$-th diagonal block and zeroes elsewhere. In this case Gupta and Robinson (2015) prove that

$$
\max _{1 \leq j \leq p}\left|\lambda_{j}\right|<1
$$

can replace the more general condition given in (5.3). Thus under (5.1), condition (2.10) is satisfied 
always if (5.3) holds for general $W_{j}$ and (5.4) holds for $W_{j}$ with 'single nonzero diagonal block' structure. Another popular normalization when the weight matrix has nonnegative elements is rownormalization, where each row of $W_{j}$ sums to 1 , thus implying $\left\|W_{j}\right\|_{R}=1$ so negligibility of $\chi_{n, R}$ follows if (5.3) holds in the general case and (5.4) holds in the 'single nonzero diagonal block' case, as illustrated for the spectral norm. More generally, if $\left\|W_{j}\right\|_{M} \leq C$, where $\|\cdot\|_{M}$ denotes a generic matrix norm, then $\mathbb{E}\left\|W_{j}\right\|_{M}^{s} \leq C$, any $s>0$. A sufficient condition for $\mathbb{E}\left\|S^{-1}\right\|_{M} \leq C$ is that $\left\|\sum_{j=1}^{p} \lambda_{j} W_{j}\right\|_{M}$ has a moment generating function.

On the other hand, all types of normalizations are not economically justified. For instance Bell and Bockstael (2000) point out that row-normalization is not justified in certain models with real estate data while Lee and $\mathrm{Yu}$ (2014) discuss problems in estimation that occur with row-normalized weight matrices and present some simulation evidence using non-normalized matrices. Thus the moment conditions implied by (2.10) and (3.5) will be different under various normalizations of $W_{j}$. The choice of normalization ultimately lies with the practitioner, but we believe (5.1) is the most attractive option. Unlike row-normalization it does not change the economic content of the spatial weight matrices because it preserves relative distances, and performs the task of stabilizing moments. Kelejian and Prucha (2010) provide an excellent discussion of normalizations and their implications, particularly for parameter spaces.

For another sufficient condition we focus on (3.6) with generic blocks $B_{m}$, where we have $p=1$ and write $W_{1}=W$ and $\lambda_{1}=\lambda$. This is sometimes referred to as a Balanced Group Interaction (BGI) setting, see e.g. Hillier and Martellosio (2013). It implies inter group independence for clustered data. Note that it does not have a 'single nonzero diagonal block' structure. We take $d, m \rightarrow \infty$, which is a combination of 'increasing domain' and 'infill' asymptotics. Suppose that the elements of $B_{m}$ are such that $\mathbb{E}\left\|B_{m}\right\|^{8}=\mathscr{O}\left(m^{\psi_{1}}\right)$ and $\mathbb{E}\left\|\left(I_{m}-\lambda B_{m}\right)^{-1}\right\|^{8}=\mathscr{O}\left(m^{\psi_{2}}\right)$, for some $\psi_{1}, \psi_{2} \geq 0$. Then $\mathbb{E}\|W\|^{8}=\mathscr{O}\left(m^{\psi_{1}}\right)$ and $\mathbb{E}\left\|S^{-1}\right\|^{8}=\mathscr{O}\left(m^{\psi_{2}}\right)$ due to their block diagonality with equal blocks, implying $\chi_{n}=\mathscr{O}\left(d^{-\frac{1}{2}} m^{\frac{\psi_{1}+\psi_{2}}{8}-\frac{1}{2}}\right)=o(1)$ always if $\psi_{1}+\psi_{2} \leq 4$ and if $m=o\left(d^{\frac{4}{\psi_{1}+\psi_{2}-4}}\right)$ when $\psi_{1}+\psi_{2}>4$.

\section{Monte Carlo}

Finite sample performance of IV, OLS and PML estimates with fixed $W_{j}$ has been examined before, see e.g. Lee (2004), Gupta and Robinson (2015), Gupta and Robinson (2018). Our aim in this section is twofold: we wish to compare performance of estimates when $W_{j}$ are stochastic as opposed to fixed, and we seek information on how estimates behave according to different spatial weight matrix structures.

\subsection{Spatial weights generated by iid random variables}

Our first design takes $p=1, k=2$ with $\lambda=0.5, \beta_{1}=1, \beta_{2}=0.7, X$ generated as independent draws from $U(0,1)$ and $u_{r}$ independent draws from a standard normal distribution in each of the 1000 replications. In this setup we examine what happens when the spatial matrices are very dense. We define $W_{1}^{*}=\operatorname{diag}\left[V_{1(m \times m)}, 0_{(m \times m)}\right], W_{2}^{*}=\operatorname{diag}\left[0_{(m \times m)}, V_{2(m \times m)}\right]$, so $n=2 m$, and generate $V_{j}$ (both of which have zeros on the diagonals) as iid replications using $\left|t_{v}\right|$ distributions with $v=$ $1,10,20,100$, and $m=48,72,144$. For the fixed design we generate such matrices once and keep them 
fixed for all 1000 replications, while for the stochastic design these are generated anew in each trial. The dependent variable is generated as $y=\lambda\left(W_{1}+W_{2}\right) y+X \beta+u$ where, for $j=1,2$, the $W_{j}$ are obtained by normalizing $W_{j}^{*}$ with the spectral norm as in (5.1). For ease of exposition we refer to the latter procedure as 'spectral-normalization'. We choose to use this block-diagonal specification to address the fact that very dense spatial weight matrices entail $h_{W}=\mathscr{O}(n)$, a situation that leads to inconsistent estimates, as noted by Lee (2004). Using a block-diagonal weight matrix with very dense blocks mitigates this problem, because now we have $h_{W}=m-1$. Instruments are selected to be $Z=\left[X,\left(W_{1}+W_{2}\right) X\right]$, while for the MLE we take $\Lambda=[-0.99,0.99]$.

We analyze absolute Monte Carlo bias, mean squared error (MSE) and size for the autoregression parameter and absolute average Monte Carlo bias, MSE and size for the regression parameters. By 'average' statistics we mean that in the rows corresponding to $\hat{\theta}$ the columns labelled $\beta$ contain entries equal to

$$
\left(\left|\operatorname{bias}\left(\hat{\beta}_{1}\right)\right|+\left|\operatorname{bias}\left(\hat{\beta}_{2}\right)\right|\right) / 2 \text {, }
$$

for instance, with similar definitions for the rows corresponding to $\tilde{\theta}$ and $\check{\theta}$. Throughout the section all Monte Carlo statistics for $\beta$ reported are averages defined like (6.1). Sizes are based on $t$-ratios, defined again as in (6.1) for $\beta$, to be compared against a nominal value of $5 \%$, computed using a critical value of 1.96 .

\section{TABLES 6.1 AND 6.2 ABOUT HERE}

Tables 6.1 and 6.2 summarize the results for stochastic and fixed $W_{j}$ respectively. The biases are quite similar for the fixed and stochastic designs for all three estimates. Out of the 72 possible comparisons, bias is greater in the fixed design for 38 cases. A similar conclusion holds for the MSE, which is higher for the fixed design in 34 cases. However, $\hat{\theta}$ seems to perform better in terms of MSE in the fixed design. Out of 24 cases, the stochastic setting yields a lower MSE on only 7 occasions, although the differences are rather small. One aspect that merits attention is the behaviour of estimates of $\lambda$ when weights are generated by $\left|t_{1}\right|$, a distribution with no defined moments. Also of interest is the behaviour more generally as $v$ increases. The bias and MSE of all estimates of $\lambda$ are high when $v=1$, indeed for the IV estimate the magnitude of the MSE is alarming when $m=72$. Increasing $m$ can sometimes help matters, possibly due to smoothing out the effect of extreme draws from a distribution with no finite moments. The theory indicates an important role played by conditions on the moments of $W_{j}$, such as Assumptions 7 and 12. Evidently the moment stabilizing transformation (5.1) that we carry out does not sufficiently improve matters when $v=1$, but increasing $v$ does so and broadly speaking helps even in improving the bias of the estimates of $\beta$, although not monotonically. The MSE improves dramatically from $v=1$ to $v>1$ but subsequently stays quite stable across the board. The findings suggest that, in practice, normalization is not enough to stabilize moments of weight matrices when the raw weights are generated by a distribution with no finite moments.

In both Tables 6.1 and 6.2, it is notable that $\tilde{\theta}$ is less biased than $\check{\theta}$ in general, even for large $m$, while the MSE is quite similar. Large $m$ means large $h_{W}$, and because the objective function for MLE differs from OLS only in a Jacobian term that is $\mathscr{O}_{p}\left(h_{W}^{-1}\right)$, this implies that there is little to choose between the two when $h_{W}$ is large. The difference in bias possibly stems from the fact that the MLE is only implicitly defined, and such estimates can sometimes have worse finite sample bias properties 
than closed form estimates. On the other hand the closeness in MSE implies that $\check{\theta}$ has much smaller variance than $\tilde{\theta}$. Empirical sizes follow the same pattern as the bias and MSE discussed above, in the sense that there is no clear dominance between the fixed and stochastic designs. The MLE tends to undersize for $\lambda$, but the problem is worse when $v=1$ and more acute in the fixed design.

The next setup considers more sparse spatial weight matrices, whereby the need for a blockdiagonal specification to mitigate denseness is dispensed with. Now we take $n=96,144,288$ (to match the sample sizes in the dense case) and generate the $n \times n$ matrix $W^{*}$ once again with iid $\left|t_{v}\right|$ elements, $v=1,10,20,100$. Next, we generate an $n \times n$ matrix with elements given by $0.75 \times$ $U(0,1)$ and round all its elements that are less than 0.5 down to zero. Finally all elements of $W^{*}$ corresponding to zeros in this matrix are set to zero. This produces a sparse $W^{*}$, in fact replacing 0.75 with a smaller (larger) factor would produce a more (less) sparse $W^{*}$. The fixed and stochastic designs differ as for the dense case. We then take $W=W^{*} /\left\|W^{*}\right\|$ and generate the dependent variable as $y=\lambda W y+X \beta+u$, with the other aspects of the experiment identical to the dense case above.

\section{TABLES 6.3 AND 6.4 ABOUT HERE}

The results are in Tables 6.3 and 6.4 for stochastic and fixed designs respectively. Biases are generally higher for $\tilde{\theta}$ than in the dense case, this being the case because sparser $W$ implies smaller $h_{W}$, cf. (3.5). Once again the case with $v=1$ produces odd results. For $n=96$ in the stochastic design, $\tilde{\lambda}$ has less bias in the sparse case than the dense one (cf. Tables 6.1 and 6.3) but this counterintuitive feature is reversed as $n$ grows, with a similar smoothing of extreme draws as discussed before. However it persists for all $n$ in the fixed design (cf. Tables 6.2 and 6.4), undoubtedly because a fresh draw of $W$ is not made in each trial and this inhibits the smoothing effect.

The biases in the stochastic design are higher in 45 cases out of 72 , as opposed to the 34 cases of Tables 6.1 and 6.2. On the other hand, the MSE is higher in the stochastic design in only 30 cases. Thus the estimates are more precise in the stochastic design, but also more biased. Sizes follow the same pattern as in Tables 6.1 and 6.2, and the undersizing for $\lambda$ with the MLE noted there is present.

The final iid setup considers extremely sparse $W$. We choose the circulant $W$ defined in (3.7) and (3.8). Once again $\omega$ is generated from iid $\left|t_{v}\right|, v=1,10,20,100$, with the fixed and stochastic designs differing because in the latter this generation happens anew in each trial. The rest of the design is as in the sparse and dense cases discussed above.

\section{TABLES 6.5 AND 6.6 ABOUT HERE}

Tables 6.5 and 6.6 contain the results for stochastic and fixed designs respectively. Theorems 3.1 and 3.2 in this paper and the results of Lee (2002) indicate that the performance of OLS estimates is poor in this situation. This is confirmed in the tables, with $\tilde{\theta}$ exhibiting unacceptably poor performance in terms of bias and size. The MSE seems more acceptable, but is much higher than that for $\check{\theta}$ and $\hat{\theta}$. Given the highly biased nature of the estimates, a low variance provides scant comfort. On the other hand, both $\hat{\theta}$ and $\check{\theta}$ perform well for both the fixed and stochastic designs. Within each table, $\check{\theta}$ is much more efficient, as is well-known to be the case for the MLE. Comparing the biases of $\hat{\theta}$ and $\ddot{\theta}$ between Tables 6.5 and 6.6 , we find that bias is bigger in the stochastic design for 31 out of 48 comparisons. A similar MSE comparison returns an equal verdict of 24 each. Thus we may conclude that there is no substantial difference between the fixed and stochastic designs. The MLE still tends 
to undersize for $\lambda$, while sizes for the IV estimate are perfectly acceptable with one exception: the $v=10$ and $n=96$ entry in Table 6.5. The online appendix contains a simulation study when the spatial weights are possibly dependent, as opposed to the iid weights considered in this section.

\subsection{Spatial weights generated by explanatory variables}

We mentioned that an important motivation of stochastic spatial weights is the fact that these are frequently generated using economic variables that are themselves stochastic. In this subsection we wish to analyse the performance of estimates when the spatial weights are generated by elements of $X$. The rank conditions for our asymptotic theorems rule out any identification problems arising from this, but we wish to study the finite sample implications of this type of feature.

We generate the spatial weight matrices as

$$
\begin{aligned}
w_{r s, 1}^{*} & =\frac{\left\|x_{r}-x_{s}\right\|}{1+\left\|x_{r}-x_{s}\right\|^{2}} \mathbb{1}(r \neq s), \\
w_{r s, 2}^{*} & =\exp \left(-\left\|x_{r}-x_{s}\right\|\right) \mathbb{1}(r \neq s),
\end{aligned}
$$

for $r, s=1, \ldots, n$ with $w_{r s, j}^{*}$ a typical element of $W_{j}^{*}, x_{r}^{\prime}$ the $r$-th row of $X$ and $n=48,96,144$. Robinson (2008) originally suggested the formula given in (6.2), though no finite-sample implications were studied in that paper. Now we generate $y=\lambda_{1} W_{1} y+\lambda_{2} W_{2} y+X \beta+u$ with $\lambda_{1}=0.2$ and $\lambda_{2}=0.3$, with $W_{j}$ denoting the spectral-normalized $W_{j}^{*}$, and search over $\Lambda=[-0.99,0.99] \times[-0.99,0.99]$ in each of the 1000 replications. Table 6.7 reports the results, reporting average statistics as in (6.1) for the two-dimensional $\lambda$ also. These paint a positive picture, in the sense that estimates exhibit bias and MSE that declines with sample size to quite acceptable levels. The bias reaches zero to two decimal places for IV for instance, and the MSE likewise reaches zero to one decimal place quite comfortably. Sizes are perfectly acceptable except for oversizing of OLS and PMLE when $n=48$, which is alleviated when $n=96$.

\section{TABLE 6.7 ABOUT HERE}

\subsection{Summary of Monte Carlo findings}

To conclude, in our design we find that there is no clear pattern of dominance between stochastic and fixed designs when spatial weight matrices are dense. On the other hand, in the sparse case the stochastic design can yield more precise estimates but with larger absolute bias than the fixed design. We also find that finite sample performance of estimates is satisfactory even when spatial weight matrices are functions of the explanatory variables that form the regression component of the model, which is anticipated from our theoretical results when multicollinearity is ruled out. However, a caveat applies to the interpretation of these findings, which are dependent on the particular way of comparing fixed and stochastic designs that we have chosen.

Finally, we note that weight matrix normalization may not stabilize moments sufficiently when raw weights come from distributions with no finite moments. Due to normalization, the theoretical conditions, such as (2.10) and (3.5), do hold in this case but finite sample performance is not up to the mark, especially so in the fixed $W_{j}$ case. A possible reason for this difference is the smoothing out of extreme draws in the stochastic design. 


\section{Conclusion and extensions}

We examined IV, OLS and PML estimates for the parameters of SAR models with stochastic weight matrices and spatial linear process dependence in disturbances. We also discussed the implications of popular weight matrix normalizations on our conditions. In the dependent disturbances setup that we consider, heteroskedasticity and autocorrelation consistent (HAC) covariance matrix estimation is an important problem. With deterministic $W_{j}$ this has been considered in the literature, see e.g. Kelejian and Prucha $(2007,2010)$ and Robinson and Thawornkaiwong (2012) for HAC estimation with SAR/SMA disturbances and disturbances satisfying Assumption 1 respectively. These approaches are straightforward to extend to the case with stochastic $W_{j}$ and using them in practice requires no change in earlier techniques.

\section{Acknowledgements}

I am grateful to co-editor Guido Kuersteiner and three anonymous referees for excellent comments that improved the paper substantially. I thank Peter Robinson for several comments and am also grateful to Nicolas Debarsy, Javier Hidalgo, Ingmar Prucha and Renata Rabovic for useful suggestions and discussions.

\section{Appendices}

\section{A Proofs of theorems}

For any matrices $\bar{F}$ and $\overline{\bar{F}}$ of equal dimension, we will write $\overline{\bar{\Delta}}^{F}=\bar{F}-\overline{\bar{F}}$.

Proof of Theorem 2.1. By (2.2) $\hat{\theta}-\theta=\bar{Q}^{-1} \overline{\bar{\Delta}}^{Q}(\hat{\theta}-\theta)-\bar{Q}^{-1} \overline{\bar{\Delta}}^{\prime} \bar{J}^{-1} q+\bar{Q}^{-1} \bar{K}^{\prime} \bar{J}^{-1} q$, so

$$
\left(I_{p+k}-\bar{Q}^{-1} \overline{\bar{\Delta}}^{Q}\right)(\hat{\theta}-\theta)=-\bar{Q}^{-1} \overline{\bar{\Delta}}^{K^{\prime}} \bar{J}^{-1} q+\bar{Q}^{-1} \bar{K}^{\prime} \bar{J}^{-1} q
$$

We first show $\overline{\bar{\Delta}}^{K}=o_{p}(1)$. Write $e_{r}$ for the $n \times 1$ vector with unity in the $r$-th position and zeroes elsewhere and $b_{i}$ for the $i$-th column of $[Z, X]$. By the law of iterated expectations, the expectation of the square of a typical $(i, j)$-th element, $i, j=1, \ldots, p_{1}+k$, of $\overline{\bar{\Delta}}^{K}=n^{-1}[Z, X]^{\prime}[B, 0]$ is

$$
n^{-2} \mathbb{E}\left(b_{i}^{\prime} G_{j} u u^{\prime} G_{j}^{\prime} b_{i}\right)=n^{-2} \mathbb{E}\left(\sum_{r, s=1}^{n} b_{i}^{\prime} G_{j} e_{r} \mathbb{E}\left(u_{r n} u_{s n}\right) e_{s}^{\prime} G_{j}^{\prime} b_{i}\right) .
$$

Now $\mathbb{E}\left(u_{r n} u_{s n}\right)=\sum_{k, l=1}^{\infty} c_{r k} c_{s l} \mathbb{E}\left(\epsilon_{k} \epsilon_{l}\right)=\sigma^{2} \sum_{k=1}^{\infty} c_{r k} c_{s k} \leq \sigma^{2}\left(\sum_{k=1}^{\infty} c_{r k}^{2}\right)^{\frac{1}{2}}\left(\sum_{k=1}^{\infty} c_{s k}^{2}\right)^{\frac{1}{2}} \leq C$, by Assumption 5 and Cauchy-Schwarz inequality, so (A.2) is bounded by $\mathrm{Cn}^{-2}$ times

$$
\mathbb{E}\left(b_{i}^{\prime} G_{j} \sum_{r, s=1}^{n} e_{r} e_{s}^{\prime} G_{j}^{\prime} b_{i}\right)=\mathbb{E}\left(b_{i}^{\prime} G_{j} \sum_{r=1}^{n} e_{r} e_{r}^{\prime} G_{j}^{\prime} b_{i}\right)=\mathbb{E}\left(b_{i}^{\prime} G_{j} G_{j}^{\prime} b_{i}\right)
$$

The term inside the expectation on the far right is bounded by $\left\|b_{i}\right\|^{2}\left\|G_{j}\right\|^{2}$ so, by the Hölder inequality 
(for expectations), (A.3) is bounded by

$$
\left(\mathbb{E}\left\|b_{i}\right\|^{2 \zeta_{1}}\right)^{\frac{1}{\zeta_{1}}}\left(\mathbb{E}\left\|G_{j}\right\|^{2 \zeta_{2}}\right)^{\frac{1}{\zeta_{2}}}
$$

The expectation inside parentheses in the first factor in (A.4) equals $\mathbb{E}\left(\sum_{r=1}^{n} a_{r i}^{2}\right)^{\zeta_{1}}$, which, by the Hölder inequality (for sums of real numbers) is bounded by

$$
n^{\zeta_{1}\left(1-\frac{1}{\zeta_{1}}\right)} \sum_{r=1}^{n} \mathbb{E}\left|a_{r i}\right|^{2 \zeta_{1}}=\mathscr{O}\left(n^{\zeta_{1}}\right)
$$

by (2.7). The second factor in (A.4) is bounded by

$$
\left\{\mathbb{E}\left(\left\|W_{j}\right\|^{2 \zeta_{2}}\left\|S^{-1}\right\|^{2 \zeta_{2}}\right)\right\}^{\frac{1}{\zeta_{2}}} \leq\left\{\left(\mathbb{E}\left\|W_{j}\right\|^{2 \zeta_{2} \zeta_{3}}\right)^{\frac{1}{\zeta_{3}}}\left(\mathbb{E}\left\|S^{-1}\right\|^{2 \zeta_{2} \zeta_{4}}\right)^{\frac{1}{\zeta_{4}}}\right\}^{\frac{1}{\zeta_{2}}}=\mathscr{O}\left(n \chi_{n}^{2}\right),
$$

once again using Hölder's inequality. Combining (A.5) and (A.6) yields

$$
\left(\mathbb{E}\left\|b_{i}\right\|^{2 \zeta_{1}}\right)^{\frac{1}{\zeta_{1}}}\left(\mathbb{E}\left\|G_{j}\right\|^{2 \zeta_{2}}\right)^{\frac{1}{\zeta_{2}}}=\mathscr{O}\left(n^{2} \chi_{n}^{2}\right)
$$

whence Markov's inequality implies that

$$
\left\|\overline{\bar{\Delta}}^{K}\right\|=\mathscr{O}_{p}\left(\chi_{n}\right)=o_{p}(1)
$$

by (2.10). By (2.8) and (A.8),

$$
\left\|\overline{\bar{\Delta}}^{Q}\right\| \leq\left\|\overline{\bar{\Delta}}^{K}\right\|\left\|\overline{\bar{J}}^{-1}\right\|\left(\left\|\overline{\bar{\Delta}}^{K}\right\|+2\|\bar{K}\|\right)=\mathscr{O}_{p}\left(\left\|\overline{\bar{\Delta}}^{K}\right\|\right)=o_{p}(1) .
$$

Finally, the expectation of the square of a typical element of $q$ is

$$
n^{-2} \mathbb{E}\left(b_{i}^{\prime} u u^{\prime} b_{i}\right)=n^{-2} \mathbb{E}\left(\sum_{r, s=1}^{n} b_{i}^{\prime} e_{r} \mathbb{E}\left(u_{r n} u_{s n}\right) e_{s}^{\prime} b_{i}\right) \leq C n^{-2} \mathbb{E}\left\|b_{i}\right\|^{2} \leq C n^{-1},
$$

so

$$
q=\mathscr{O}_{p}\left(n^{-\frac{1}{2}}\right) .
$$

Using (A.8), (A.9), (A.10) and (2.8) in (A.1) we obtain the desired result.

Proof of Theorem 2.2. In view of (A.1), (A.8), (A.9) and (A.10) it suffices to show $n^{\frac{1}{2}} q \stackrel{d}{\longrightarrow} \mathcal{N}\left(0, \sigma^{2} \Phi\right)$. The proof now follows Robinson and Thawornkaiwong (2012), who modified a proof of Robinson and Hidalgo (1997). Write $d=d_{n}=n^{-\frac{1}{2}} \sum_{r=1}^{n} a_{r} u_{r}=n^{-\frac{1}{2}} \sum_{l=1}^{\infty} f_{l} \epsilon_{l}$, where $f_{l}=f_{l n}=\sum_{r=1}^{n} a_{r} c_{r l}$. By Lemma A1 of Robinson and Thawornkaiwong (2012), there exists a sequence $N=N_{n}$, increasing in $n$ without bound, such that $d-d_{N}=o_{p}(1)$, where $d_{N}=n^{-\frac{1}{2}} \sum_{l=1}^{N} f_{l} \epsilon_{l}$. Writing $E=E_{n}=n^{-1} \sum_{l=1}^{N} f_{l} f_{l}^{\prime}$, again Lemma A1 of Robinson and Thawornkaiwong (2012) implies that $E \stackrel{p}{\longrightarrow} \sigma^{2} \Phi$, by Assumption 6. Let $\alpha \in \mathbb{R}^{p+k}$ such that $\|\alpha\|^{2}=1$ and $c_{N}=\alpha^{\prime} E^{-\frac{1}{2}} d_{N}$, $v_{l}=v_{l n}=n^{-\frac{1}{2}} \alpha^{\prime} E^{-\frac{1}{2}} f_{l}$. Then $c_{N}=\sum_{l=1}^{N} v_{l} \epsilon_{l}$, and Assumption 6 implies that $\left\{f_{l} \epsilon_{l}, 1 \leq l \leq N\right\}$ is a martingale difference sequence for each $N \geq 1$. We show $c_{N} \stackrel{d}{\longrightarrow} \mathcal{N}(0,1)$, conditional on $X, z_{r}$ and $W_{j}, j=1, \ldots, p$, which follows by Theorem 2 of Scott (1973) if, conditional on $X, z_{r}$ and $W_{j}$, 
$j=1, \ldots, p$, as $n \rightarrow \infty$,

$$
\mathbb{E}\left(\sum_{l=1}^{N} v_{l}^{2} \epsilon_{l}^{2} \mid \epsilon_{j}, j<l\right) \stackrel{p}{\rightarrow} 1,
$$

and for all $\xi>0$,

$$
\mathbb{E}\left(\sum_{l=1}^{N} v_{l}^{2} \mathbb{E}\left(\epsilon_{l}^{2} \mathbb{1}\left(\left|v_{l} \epsilon_{l}\right|>\xi\right) \mid z_{r}, X, W_{1}, \ldots, W_{p}\right)\right) \rightarrow 0 .
$$

The LHS of (A.11) equals 1, while the LHS of (A.12) is bounded by

$$
\max _{1 \leq l \leq N} \mathbb{E}\left\{\epsilon_{l}^{2} \mathbb{1}\left(\epsilon_{l}^{2}>\frac{\xi^{2}}{\max _{1 \leq l \leq N} v_{l}^{2}}\right)\right\} \mathbb{E}\left(\sum_{l=1}^{N} v_{l}^{2}\right) .
$$

By Assumption 5, it suffices to show that $\max _{1 \leq l \leq N} v_{l}^{2}=o_{p}(1)$ as $n \rightarrow \infty$, as the rightmost factor equals 1. Now, $\max _{1 \leq l \leq N} v_{l}^{2} \leq n^{-1}\left\|E^{-\frac{1}{2}}\right\|^{2} \sup _{l \geq 1}\left\|\sum_{r=1}^{n} a_{r} c_{r l}\right\|^{2}=o_{p}(1)$ by Assumptions 4,5 and 6.

Proof of Theorem 3.1. By (3.1) $\tilde{\theta}-\theta=\bar{L}^{-1} \overline{\bar{\Delta}}^{L}(\tilde{\theta}-\theta)+\bar{L}^{-1} w$, so

$$
\left(I_{p+k}-\bar{L}^{-1} \overline{\bar{\Delta}}^{L}\right)(\tilde{\theta}-\theta)=\bar{L}^{-1} w .
$$

Note that $\|\ell\| \leq\left\|n^{-1}[A, X]^{\prime} u\right\|+\left\|n^{-1}[B, 0]^{\prime} u\right\|$, where the first term on the RHS is readily shown to be negligible, as we deduced (A.10), but using (3.2) in Assumption 8 instead of (2.7) in Assumption 4 because here $Z$ is replaced by $A$. Next $n^{-1}[B, 0]^{\prime} u=o_{p}(1)$ by (3.5) and Lemma B.3, so that $\ell=o_{p}(1)$. It remains to prove that $\overline{\bar{\Delta}}^{L}=o_{p}(1)$, for which first note that

$$
\left\|\overline{\bar{\Delta}}^{L}\right\| \leq n^{-1}\|B\|^{2}+2 n^{-1}\left\|[A, X]^{\prime}[B, 0]\right\| .
$$

The first term on the RHS is $\mathscr{O}_{p}\left(\pi_{n}\right)$, by the proof of Lemma B.3, and is negligible by (3.5). The second term on the RHS is bounded exactly like $\left\|\overline{\bar{\Delta}}^{K}\right\|=n^{-1}[Z, X]^{\prime}[B, 0]$ in the proof of Theorem 2.1, but again using (3.2) in Assumption 8 instead of (2.7) in Assumption 4 because here $Z$ is replaced by $A$, see also the proof of Theorem 4.1 in Gupta and Robinson (2015). Therefore

$$
\left\|\overline{\bar{\Delta}}^{L}\right\|=\mathscr{O}_{p}\left(\max \left\{\pi_{n}, \chi_{n}\right\}\right)=o_{p}(1),
$$

and the theorem is proved.

Proof of Theorem 3.2. We claim that it is sufficient to prove

$$
n^{-\frac{1}{2}}[A, X]^{\prime} u \stackrel{d}{\rightarrow} \mathcal{N}(0, \Psi),
$$

for which, in view of (A.13), (A.15) and $\ell=n^{-1}[A, X]^{\prime} u+n^{-1}[B, 0]^{\prime} u$ it is enough to show that

$$
n^{-\frac{1}{2}}[B, 0]^{\prime} u=o_{p}(1)
$$

To show (A.17), we can exploit Assumption 9 to obtain a sharper bound for $[B, 0]^{\prime} u$ than the one used 
in the proof of Theorem 3.1, where Assumption 5 sufficed. Indeed, by Lemma B.4, $n^{-\frac{1}{2}}[B, 0]^{\prime} u=$ $\mathscr{O}_{p}\left(n^{\frac{1}{2}}\left(\mathbb{E}\left\|S^{\prime-1}\right\|_{R}^{2 \zeta_{12}}\right)^{\frac{1}{2 \zeta_{12}}} h_{W}^{-1}\right)=o_{p}(1)$, by (3.11). The proof of (A.16) follows exactly as the proof of asymptotic normality of $n^{\frac{1}{2}} q$ in Theorem 2.2, and we omit the details, noting only that here $A$ replaces $Z$, and Assumption 10 replaces Assumption 6.

Proof of Theorem 4.1. We show $\check{\gamma} \stackrel{p}{\rightarrow} \gamma_{0}$, whence $\breve{\beta} \stackrel{p}{\rightarrow} \beta_{0}$ and $\check{\sigma}^{2} \stackrel{p}{\rightarrow} \sigma_{0}^{2}$ follow from (4.2) and (4.3) respectively. First note that

$$
\mathcal{L}(\gamma)-\mathcal{L}=\log \bar{\sigma}^{2}(\gamma) / \bar{\sigma}^{2}-n^{-1} \log \left|T^{\prime}(\lambda) \Xi(\tau)^{-1} T(\lambda) \Xi\right|=\log \bar{\sigma}^{2}(\gamma) / \sigma^{2}(\gamma)-\log \bar{\sigma}^{2} / \sigma_{0}^{2}+\log r(\gamma),
$$

where recall that $\sigma^{2}(\gamma)=n^{-1} \sigma_{0}^{2} \operatorname{tr}\left(T^{\prime}(\lambda) \Xi(\tau)^{-1} T(\lambda) \Xi\right), \bar{\sigma}^{2}=\bar{\sigma}^{2}\left(\gamma_{0}\right)=n^{-1} u^{\prime} \Xi^{\prime-\frac{1}{2}} M \Xi^{-\frac{1}{2}} u$, using (4.3) and also $r(\gamma)=n^{-1} \operatorname{tr}\left(T^{\prime}(\lambda) \Xi(\tau)^{-1} T(\lambda) \Xi\right) /\left|T^{\prime}(\lambda) \Xi(\tau)^{-1} T(\lambda) \Xi\right|^{1 / n}$. From $(2.6), \bar{\sigma}^{2}(\gamma)=$ $n^{-1}\left\{S^{-1^{\prime}}\left(X \beta_{0}+u\right)\right\}^{\prime} S^{\prime}(\lambda) \Xi(\tau)^{\prime-\frac{1}{2}} M(\tau) \Xi(\tau)^{-\frac{1}{2}} S(\lambda) S^{-1}\left(X \beta_{0}+u\right)=c_{1}(\gamma)+c_{2}(\gamma)+c_{3}(\gamma)$, where

$$
\begin{aligned}
c_{1}(\gamma) & =n^{-1} \beta_{0}^{\prime} X^{\prime} T^{\prime}(\lambda) \Xi(\tau)^{\prime-\frac{1}{2}} M(\tau) \Xi(\tau)^{-\frac{1}{2}} T(\lambda) X \beta_{0}, \\
c_{2}(\gamma) & =n^{-1} \sigma_{0}^{2} \operatorname{tr}\left(T^{\prime}(\lambda) \Xi(\tau)^{\prime-\frac{1}{2}} M(\tau) \Xi(\tau)^{-\frac{1}{2}} T(\lambda) \Xi\right), \\
c_{3}(\gamma) & =n^{-1} \operatorname{tr}\left(T^{\prime}(\lambda) \Xi(\tau)^{\prime-\frac{1}{2}} M(\tau) \Xi(\tau)^{-\frac{1}{2}} T(\lambda)\left(u u^{\prime}-\sigma_{0}^{2} \Xi\right)\right) \\
& +2 n^{-1} \beta_{0}^{\prime} X^{\prime} T^{\prime}(\lambda) \Xi(\tau)^{\prime-\frac{1}{2}} M(\tau) \Xi(\tau)^{-\frac{1}{2}} T(\lambda) u .
\end{aligned}
$$

Then

$$
\begin{aligned}
\log \frac{\bar{\sigma}^{2}(\gamma)}{\sigma^{2}(\gamma)} & =\log \frac{\bar{\sigma}^{2}(\gamma)}{\left(c_{1}(\gamma)+c_{2}(\gamma)\right)}+\log \frac{c_{1}(\gamma)+c_{2}(\gamma)}{\sigma^{2}(\gamma)} \\
& =\log \left(1+\frac{c_{3}(\gamma)}{c_{1}(\gamma)+c_{2}(\gamma)}\right)+\log \left(1+\frac{c_{1}(\gamma)-f(\gamma)}{\sigma^{2}(\gamma)}\right)
\end{aligned}
$$

where $f(\gamma)=n^{-1} \sigma_{0}^{2} \operatorname{tr}\left(\Xi^{\prime \frac{1}{2}} T^{\prime}(\lambda) \Xi(\tau)^{\prime-\frac{1}{2}}\left(I_{n}-M(\tau)\right) \Xi(\tau)^{-\frac{1}{2}} T(\lambda) \Xi^{\frac{1}{2}}\right)$. Then (A.18) implies

$$
\begin{aligned}
P\left(\left\|\hat{\gamma}-\gamma_{0}\right\| \in \overline{\mathcal{N}}^{\gamma}(\eta)\right) & =P\left(\inf _{\gamma \in \overline{\mathcal{N}}^{\gamma}(\eta)} \mathcal{L}(\gamma)-\mathcal{L} \leq 0\right) \\
\leq & P\left(\log \left(1+\sup _{\gamma \in \overline{\mathcal{N}}^{\gamma}(\eta)}\left|\frac{c_{3}(\gamma)}{c_{1}(\gamma)+c_{2}(\gamma)}\right|\right)+\left|\log \left(\bar{\sigma}^{2} / \sigma_{0}^{2}\right)\right|\right. \\
& \left.\geq \inf _{\gamma \in \overline{\mathcal{N}}^{\gamma}(\eta)}\left(\log \left(1+\frac{c_{1}(\gamma)-f(\gamma)}{\sigma^{2}(\gamma)}\right)+\log r(\gamma)\right)\right),
\end{aligned}
$$

where recall that $\overline{\mathcal{N}}^{\gamma}(\eta)=\Gamma \backslash \mathcal{N}^{\gamma}(\eta), \mathcal{N}^{\gamma}(\eta)=\left\{\gamma:\left\|\gamma-\gamma_{0}\right\|<\eta\right\} \cap \Gamma$. Because $\bar{\sigma}^{2} / \sigma_{0}^{2} \stackrel{p}{\rightarrow} 1$, the property $\log (1+x)=x+o(x)$ as $x \rightarrow 0$ implies that it is sufficient to show that

$$
\begin{array}{r}
\sup _{\gamma \in \overline{\mathcal{N}}^{\gamma}(\eta)}\left|\frac{c_{3}(\gamma)}{c_{1}(\gamma)+c_{2}(\gamma)}\right| \stackrel{p}{\longrightarrow} 0, \\
\sup _{\gamma \in \overline{\mathcal{N}}^{\gamma}(\eta)}\left|\frac{f(\gamma)}{\sigma^{2}(\gamma)}\right| \stackrel{p}{\longrightarrow} 0,
\end{array}
$$




$$
P\left(\inf _{\gamma \in \overline{\mathcal{N}}^{\gamma}(\eta)}\left\{\frac{c_{1}(\gamma)}{\sigma^{2}(\gamma)}+\log r(\gamma)\right\}>0\right) \longrightarrow 1
$$

Because $\overline{\mathcal{N}}^{\gamma}(\eta) \subseteq\left\{\Lambda \times \overline{\mathcal{N}}^{\tau}(\eta / 2)\right\} \cup\left\{\overline{\mathcal{N}}^{\lambda}(\eta / 2) \times \mathcal{T}\right\}$, we have

$$
\begin{aligned}
& P\left(\inf _{\gamma \in \mathcal{\mathcal { N }}^{\gamma}(\eta)}\left\{\frac{c_{1}(\gamma)}{\sigma^{2}(\gamma)}+\log r(\gamma)\right\}>0\right) \geq P\left(\min \left\{\inf _{\Lambda \times \overline{\mathcal{N}}^{\tau}(\eta / 2)} \frac{c_{1}(\gamma)}{\sigma^{2}(\gamma)}, \inf _{\overline{\mathcal{N}}^{\lambda}(\eta / 2)} \log r(\gamma)\right\}>0\right)
\end{aligned}
$$

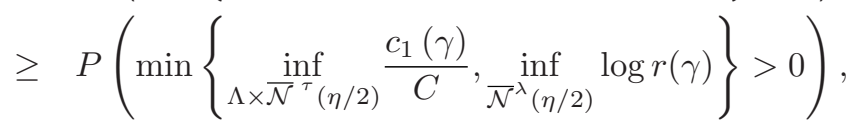

from Assumption 15, whence Assumptions 16 and 17 imply (A.21). Again using Assumption 15, uniformly in $\gamma,\left|f(\gamma) / \sigma^{2}(\gamma)\right|=\mathscr{O}_{p}(|f(\gamma)|)$ and

$$
\begin{aligned}
|f(\gamma)| & =\mathscr{O}_{p}\left(\operatorname{tr}\left(\Xi^{\prime \frac{1}{2}} T^{\prime}(\lambda) \Xi(\tau)^{-1} X\left(X^{\prime} \Xi(\tau)^{-1} X\right)^{-1} X^{\prime} \Xi(\tau)^{-1} T(\lambda) \Xi^{\frac{1}{2}}\right) / n\right) \\
& =\mathscr{O}_{p}\left(\operatorname{tr}\left(\Xi^{\prime \frac{1}{2}} T^{\prime}(\lambda) \Xi(\tau)^{-1} X X^{\prime} \Xi(\tau)^{-1} T(\lambda) \Xi^{\frac{1}{2}}\right) / n^{2}\right)=\mathscr{O}_{p}\left(\left\|X^{\prime} \Xi(\tau)^{-1} T(\lambda) \Xi^{\frac{1}{2}} / n\right\|_{F}^{2}\right) \\
& =\mathscr{O}_{p}\left(\|X / n\|_{F}^{2} \bar{\varphi}^{2}\left(\Xi(\tau)^{-1}\right)\|T(\lambda)\|^{2}\left\|\Xi^{\frac{1}{2}}\right\|^{2}\right)=\mathscr{O}_{p}\left(\|X / n\|_{F}^{2}\|T(\lambda)\|^{2} \bar{\varphi}(\Xi) / \underline{\varphi}^{2}(\Xi(\tau))\right) \\
& =\mathscr{O}_{p}\left(\|T(\lambda)\|^{2} / n\right),
\end{aligned}
$$

where we have twice made use of the inequality

$$
\|A B\|_{F} \leq\|A\|_{F}\|B\|
$$

for generic multiplication compatible matrices $A$ and B. (A.20) now follows by Assumption 12 and compactness of $\Lambda$ because $T(\lambda)=I_{n}+\sum_{j=1}^{p}\left(\lambda_{0 j}-\lambda_{j}\right) G_{j}$. Finally consider (A.19). We first prove pointwise convergence. For any fixed $\gamma \in \overline{\mathcal{N}}^{\gamma}(\eta)$ and large enough $n$, Assumptions 15 and 17 imply

$$
\begin{aligned}
& \left\{c_{1}(\gamma)\right\}^{-1}=\mathscr{O}_{p}\left(\left\|\beta_{0}\right\|^{-2}\right)=\mathscr{O}_{p}(1) \\
& \left\{c_{2}(\gamma)\right\}^{-1}=\mathscr{O}_{p}(1),
\end{aligned}
$$

because $\left\{n^{-1} \sigma_{0}^{2} \operatorname{tr}\left(\Xi^{\prime \frac{1}{2}} T^{\prime}(\lambda) \Xi(\tau)^{-1} T(\lambda) \Xi^{\frac{1}{2}}\right)\right\}^{-1}=\mathscr{O}_{p}(1)$ and, proceeding like in the bound for $|f(\gamma)|, \operatorname{tr}\left(\Xi^{\prime \frac{1}{2}} T^{\prime}(\lambda) \Xi(\tau)^{\prime-\frac{1}{2}}(I-M(\tau)) \Xi(\tau)^{-\frac{1}{2}} T(\lambda) \Xi^{\frac{1}{2}}\right)=\mathscr{O}_{p}\left(\|T(\lambda)\|^{2} / n\right)=\mathscr{O}_{p}(1 / n)$. In fact it is worth noting for the equicontinuity argument presented later that Assumptions 15 and 17 actually imply that (A.24) and (A.25) hold uniformly over $\overline{\mathcal{N}}^{\gamma}(\eta)$, a property not needed for the present pointwise arguments. Thus $c_{3}(\gamma) /\left(c_{1}(\gamma)+c_{2}(\gamma)\right)=\mathscr{O}_{p}\left(\left|c_{3}(\gamma)\right|\right)$ where, writing $\mathfrak{B}(\gamma)=$ $T^{\prime}(\lambda) \Xi(\tau)^{\prime-\frac{1}{2}} M(\tau) \Xi(\tau)^{-\frac{1}{2}} T(\lambda)$ with typical element $\mathfrak{b}_{r s}(\gamma), r, s=1, \ldots, n, c_{3}(\gamma)$ has mean 0 and variance

$$
\mathscr{O}_{p}\left(\frac{\|\mathfrak{B}(\gamma) \Xi\|_{F}^{2}}{n^{2}}+\frac{\sum_{r, s, t, v=1}^{n} \mathfrak{b}_{r s}(\gamma) \mathfrak{b}_{t v}(\gamma) \kappa_{r s t v}}{n^{2}}+\frac{\left\|\beta_{0}^{\prime} X^{\prime} \mathfrak{B}(\gamma) \Xi^{\frac{1}{2}}\right\|^{2}}{n^{2}}\right),
$$

with $\kappa_{r s t v}$ denoting the fourth cumulant of $u_{r}, u_{s}, u_{t}, u_{v}, r, s, t, v=1, \ldots, n$. Under the linear process 
assumed in Assumption 1 it is known that

$$
\sum_{r, s, t, v=1}^{n} \kappa_{r s t v}^{2}=\mathscr{O}(n)
$$

Using (A.23) and Assumptions 12 and 13, the first term in parentheses in (A.26) is

$$
\begin{aligned}
\mathscr{O}_{p}\left(\|\mathfrak{B}(\gamma)\|_{F}^{2} \bar{\varphi}^{2}(\Xi) / n^{2}\right) & =\mathscr{O}_{p}\left(\|T(\lambda)\|_{F}^{2}\left\|\Xi(\tau)^{-\frac{1}{2}}\right\|^{4}\|M(\tau)\|^{2}\|T(\lambda)\|^{2} / n^{2}\right) \\
& =\mathscr{O}_{p}\left(\|T(\lambda)\|^{4} / n \underline{\varphi}^{2}(\Xi(\tau))\right)=\mathscr{O}_{p}\left(\|T(\lambda)\|^{4} / n\right),
\end{aligned}
$$

while the second is similarly

$$
\mathscr{O}_{p}\left\{\left(\|\mathfrak{B}(\gamma)\|_{F}^{2} / n\right)\left(\sum_{r, s, t, v=1}^{n} \kappa_{r s t v}^{2} / n^{2}\right)^{\frac{1}{2}}\right\}=o_{p}\left(\|T(\lambda)\|^{4}\right),
$$

using (A.27). Finally, using (3.2), the third term in parentheses in (A.26) is

$$
\mathscr{O}_{p}\left(\|\mathfrak{B}(\gamma)\|^{2} / n\right)=\mathscr{O}_{p}\left(\|T(\lambda)\|^{4} / n\right) .
$$

By compactness of $\Lambda$ and Assumption 12, (A.28), (A.29) and (A.30) are negligible, thus pointwise convergence is established.

Uniform convergence will follow from an equicontinuity argument. First, for arbitrary $\varepsilon>0$ we can find points $\gamma_{*}=\left(\lambda_{*}^{\prime}, \tau_{*}^{\prime}\right)^{\prime}$, possibly infinitely many, such that the neighbourhoods $\left\|\gamma-\gamma^{*}\right\|<\varepsilon$ form an open cover of $\overline{\mathcal{N}}^{\gamma}(\eta)$. Since $\Gamma$ is compact any open cover has a finite subcover and thus we may in fact choose finitely many $\gamma_{*}=\left(\lambda_{*}^{\prime}, \tau_{*}^{\prime}\right)^{\prime}$, whence it suffices to prove

$$
\sup _{\left\|\gamma-\gamma_{*}\right\|<\varepsilon}\left|\frac{c_{3}(\gamma)}{c_{1}(\gamma)+c_{2}(\gamma)}-\frac{c_{3}\left(\gamma_{*}\right)}{c_{1}\left(\gamma_{*}\right)+c_{2}\left(\gamma_{*}\right)}\right| \stackrel{p}{\longrightarrow} 0 .
$$

Proceeding as in Gupta and Robinson (2018), we denote the two components of $c_{3}(\gamma)$ by $c_{31}(\gamma)$, $c_{32}(\gamma)$, and are left with establishing the negligibility of

$$
\frac{\left|c_{31}(\gamma)-c_{31}\left(\gamma_{*}\right)\right|}{c_{2}(\gamma)}+\frac{\left|c_{32}(\gamma)-c_{32}\left(\gamma_{*}\right)\right|}{c_{1}(\gamma)}+\frac{\left|c_{3}\left(\gamma_{*}\right)\right|}{c_{1}(\gamma) c_{1}\left(\gamma_{*}\right)}\left|c_{1}\left(\gamma_{*}\right)-c_{1}(\gamma)\right|+\frac{\left|c_{3}\left(\gamma_{*}\right)\right|}{c_{2}(\gamma) c_{2}\left(\gamma_{*}\right)}\left|c_{2}\left(\gamma_{*}\right)-c_{2}(\gamma)\right|,
$$

uniformly on $\left\|\gamma-\gamma_{*}\right\|<\varepsilon$. By the fact that (A.24) and (A.25) hold uniformly over $\Gamma$, we first consider only the numerators in the first two terms in (A.31). As in the proof of Theorem 1 of Delgado and Robinson (2015), (A.23) implies that $\mathbb{E}\left(\sup _{\left\|\gamma-\gamma_{*}\right\|<\varepsilon}\left|c_{31}(\gamma)-c_{31}\left(\gamma_{*}\right)\right|\right)$ is bounded by $n^{-1}\left(\mathbb{E}\|u\|^{2}+\sigma_{0}^{2} \operatorname{tr} \Xi\right) \sup _{\left\|\gamma-\gamma_{*}\right\|<\varepsilon}\left\|\mathfrak{B}(\gamma)-\mathfrak{B}\left(\gamma_{*}\right)\right\|=\mathscr{O}_{p}\left(\sup _{\left\|\gamma-\gamma_{*}\right\|<\varepsilon}\left\|\mathfrak{B}(\gamma)-\mathfrak{B}\left(\gamma_{*}\right)\right\|\right)$, because $\mathbb{E}\|u\|^{2}=\mathscr{O}(n)$ and $\operatorname{tr} \Xi=\mathscr{O}(n) \cdot \mathfrak{B}(\gamma)-\mathfrak{B}\left(\gamma_{*}\right)$ can be written as

$$
\begin{aligned}
& \left(T(\lambda)-T\left(\lambda_{*}\right)\right)^{\prime} \Xi(\tau)^{\prime-\frac{1}{2}} M(\tau) \Xi(\tau)^{-\frac{1}{2}} T(\lambda)+T\left(\lambda_{*}\right)^{\prime} \Xi^{\prime}\left(\tau_{*}\right) M\left(\tau_{*}\right) \Xi\left(\tau_{*}\right)^{-\frac{1}{2}}\left(T(\lambda)-T\left(\lambda_{*}\right)\right) \\
+ & T^{\prime}\left(\lambda_{*}\right)\left(\Xi(\tau)^{\prime-\frac{1}{2}} M(\tau) \Xi(\tau)^{-\frac{1}{2}}-\Xi\left(\tau_{*}\right)^{\prime-\frac{1}{2}} M\left(\tau_{*}\right) \Xi\left(\tau_{*}\right)^{-\frac{1}{2}}\right) T(\lambda),
\end{aligned}
$$


which, by the triangle inequality, has spectral norm bounded by

$$
\begin{aligned}
& \left\|T(\lambda)-T\left(\lambda_{*}\right)\right\|\left(\left\|\Xi(\tau)^{-\frac{1}{2}}\right\|^{2}\|T(\lambda)\|+\left\|\Xi\left(\tau_{*}\right)^{-\frac{1}{2}}\right\|^{2}\left\|T\left(\lambda_{*}\right)\right\|\right) \\
+ & \left\|T\left(\lambda_{*}\right)\right\|\left\|\Xi(\tau)^{\prime-\frac{1}{2}} M(\tau) \Xi(\tau)^{-\frac{1}{2}}-\Xi\left(\tau_{*}\right)^{\prime-\frac{1}{2}} M\left(\tau_{*}\right) \Xi\left(\tau_{*}\right)^{-\frac{1}{2}}\right\|\|T(\lambda)\| \\
= & \mathscr{O}_{p}\left(\left\|T(\lambda)-T\left(\lambda_{*}\right)\right\|+\left\|\Xi(\tau)^{\prime-\frac{1}{2}} M(\tau) \Xi(\tau)^{-\frac{1}{2}}-\Xi\left(\tau_{*}\right)^{\prime-\frac{1}{2}} M\left(\tau_{*}\right) \Xi\left(\tau_{*}\right)^{-\frac{1}{2}}\right\|\right) .
\end{aligned}
$$

By Assumption 12 the first term in parentheses on the right side of (A.33) is bounded uniformly on $\left\|\gamma-\gamma_{*}\right\|<\varepsilon$ by

$$
\sum_{j=1}^{p}\left|\lambda_{j}-\lambda_{* j}\right|\left\|G_{j}\right\| \leq \max _{j=1, \ldots, p}\left\|G_{j}\right\|\left\|\lambda-\lambda_{*}\right\|=\mathscr{O}_{p}(\varepsilon)
$$

while because $\Xi(\tau)^{\prime-\frac{1}{2}} M(\tau) \Xi(\tau)^{-\frac{1}{2}}=n^{-1} \Xi(\tau)^{-1} X\left(n^{-1} X^{\prime} \Xi(\tau)^{-1} X\right)^{-1} X^{\prime} \Xi(\tau)^{-1}$ for any $\tau \in \mathcal{T}$, the second one can be decomposed into terms with bounds typified by

$$
\begin{aligned}
& n^{-1}\left\|\Xi(\tau)^{-1}-\Xi\left(\tau_{*}\right)^{-1}\right\|\|X\|^{2}\left\|\left(n^{-1} X^{\prime} \Xi(\tau)^{-1} X\right)^{-1}\right\|\left\|\Xi(\tau)^{-1}\right\|^{2} \\
\leq & n^{-1}\left\|\Xi(\tau)-\Xi\left(\tau_{*}\right)\right\|\|X\|^{2}\left\|\left(n^{-1} X^{\prime} \Xi(\tau)^{-1} X\right)^{-1}\right\|\left\|\Xi(\tau)^{-1}\right\|^{3}\left\|\Xi\left(\tau_{*}\right)^{-1}\right\| \\
= & \mathscr{O}_{p}\left(\left\|\Xi(\tau)-\Xi\left(\tau_{*}\right)\right\|\right)=\mathscr{O}_{p}(\varepsilon),
\end{aligned}
$$

uniformly on $\left\|\gamma-\gamma_{*}\right\|<\varepsilon$, by Assumptions $13,14,18,(3.2)$ and the inequality $\|A\| \leq\|A\|_{F}$ for a generic matrix $A$, so that

$$
\sup _{\left\|\gamma-\gamma_{*}\right\|<\varepsilon}\left\|\mathfrak{B}(\gamma)-\mathfrak{B}\left(\gamma_{*}\right)\right\|=\mathscr{O}_{p}(\varepsilon) .
$$

Thus equicontinuity of the first term in (A.31) follows because $\varepsilon$ is arbitrary. The equicontinuity of the second term in (A.31) follows in much the same way. Indeed $\sup _{\left\|\gamma-\gamma_{*}\right\|<\varepsilon} c_{32}(\gamma)-c_{32}\left(\gamma_{*}\right)=$ $2 n^{-1} \beta_{0}^{\prime} X^{\prime} \sup _{\left\|\gamma-\gamma_{*}\right\|<\varepsilon}\left(\mathfrak{B}(\gamma)-\mathfrak{B}\left(\gamma_{*}\right)\right) u=\mathscr{O}_{p}\left(\sup _{\left\|\gamma-\gamma_{*}\right\|<\varepsilon}\left\|\mathfrak{B}(\gamma)-\mathfrak{B}\left(\gamma_{*}\right)\right\|\right)=\mathscr{O}_{p}(\varepsilon)$, using earlier arguments and (A.35). Because $c_{1}(\gamma)$ is bounded and bounded away from zero in probability (see A.24) for sufficiently large $n$ and all $\gamma \in \overline{\mathcal{N}}^{\gamma}(\eta)$, the third term in (A.31) may be bounded by $\left|c_{3}\left(\gamma_{*}\right)\right| / c_{1}\left(\gamma_{*}\right)\left(1+c_{1}\left(\gamma_{*}\right) / c_{1}(\gamma)\right) \stackrel{p}{\longrightarrow} 0$, convergence being uniform on $\left\|\gamma-\gamma_{*}\right\|<\varepsilon$ by pointwise convergence of $c_{3}(\gamma) /\left(c_{1}(\gamma)+c_{2}(\gamma)\right)$, cf. Gupta and Robinson (2018). The uniform convergence to zero of the fourth term in (A.31) follows in identical fashion, because $c_{2}(\gamma)$ is bounded and bounded away from zero (see (A.25)) in probability for sufficiently large $n$ and all $\gamma \in \overline{\mathcal{N}}^{\gamma}(\eta)$. This concludes the proof.

Proof of Theorem 4.2. We first introduce the derivative of (4.1) evaluated at $\left(\varrho_{0}^{\prime}, \sigma_{0}^{2}\right)^{\prime}$ :

$$
\begin{aligned}
& d=-n^{-1} \sigma_{0}^{-2}\left[2\left(u^{\prime} G_{1}^{\prime} \Xi^{-1} u-\sigma_{0}^{2} \operatorname{tr} G_{1}, \ldots, u^{\prime} G_{p}^{\prime} \Xi^{-1} u-\sigma_{0}^{2} \operatorname{tr} G_{p}, 0^{\prime}, 0^{\prime}\right)^{\prime}\right. \\
& +\quad\left(0^{\prime}, 0^{\prime}, u^{\prime} \Xi^{-1} \Xi_{1} \Xi^{-1} u-\sigma_{0}^{2} \operatorname{tr}\left(\Xi^{-1} \Xi_{1}\right), \ldots, u^{\prime} \Xi^{-1} \Xi_{q} \Xi^{-1} u-\sigma_{0}^{2} \operatorname{tr}\left(\Xi^{-1} \Xi_{q}\right)\right)^{\prime} \\
& \left.+\quad 2\left(u^{\prime} \Xi^{-1} A, u^{\prime} \Xi^{-1} X, 0^{\prime}\right)^{\prime}\right] .
\end{aligned}
$$

The first part of the proof consists of proving that

$$
n^{\frac{1}{2}} N d \stackrel{d}{\longrightarrow} \mathcal{N}(0, \Delta) .
$$


Proving (A.37) requires the establishment of asymptotic normality for normalized linear combinations of the LHS whence the required multivariate normality follows via the Cramer-Wold device, as usual. From (A.36) we observe that such a linear combination will have terms that are linear in $u$ and quadratic in $u$, and our aim of establishing asymptotic normality will be achieved by writing these terms as sums of martingale differences in the $\epsilon_{l}$. First, notice that $d=\sum_{l=1}^{\infty} w_{l}$ with $w_{l}=w_{1 l}+w_{2 l}+w_{3 l}$ and $w_{1 l}=-2 n^{-1} \sigma_{0}^{-2}\left(\nu_{l l}^{\prime \lambda}\left(\epsilon_{l}^{2}-\sigma_{0}^{2}\right)+2 \mathbb{1}(l \geq 2) \epsilon_{l} \sum_{k=1}^{l-1} \nu_{l k}^{\prime \lambda} \epsilon_{k}, 0^{\prime}, 0^{\prime}\right)^{\prime}, w_{2 l}=$ $-2 n^{-1} \sigma_{0}^{-2}\left(c_{l}^{\prime} \Xi^{-1} A \epsilon_{l}, c_{l}^{\prime} \Xi^{-1} X \epsilon_{l}, 0^{\prime}\right)^{\prime}, w_{3 l}=-n^{-1} \sigma_{0}^{-2}\left(0^{\prime}, 0^{\prime}, \nu_{l l}^{\prime \tau}\left(\epsilon_{l}^{2}-\sigma_{0}^{2}\right)+2 \mathbb{1}(l \geq 2) \epsilon_{l} \sum_{k=1}^{l-1} \nu_{l k}^{\prime \tau} \epsilon_{k}\right)^{\prime}$, where $\nu_{l k}^{\lambda}$ is a $p \times 1$ vector with $j$-th element $c_{l}^{\prime} G_{j} \Xi^{-1} c_{k}$ and $\nu_{l k}^{\tau}$ is a $q \times 1$ vector with $j$-th element $c_{l}^{\prime} \Xi^{-1} \Xi_{j} \Xi^{-1} c_{k} l, k \geq 1$. Notice that here $k$ is used as an index, without risk of confusion with the dimension of $\beta$. We first show that

$$
n^{\frac{1}{2}} N d_{*} \stackrel{p}{\longrightarrow} 0
$$

where $d_{*}=d-d_{L}, d_{L}=\sum_{l=1}^{L} w_{l}$ and $L=L_{n}$ is a positive integer sequence that is increasing in $n$. All expectations in the sequel are taken conditional on $W_{j}$ and $X, j=1, \ldots, p$.

By Chebyshev's inequality proving

$$
\mathbb{E}\left\|n^{\frac{1}{2}} N d_{*}\right\|^{2} \stackrel{p}{\longrightarrow} 0
$$

is sufficient to establish (A.38). The LHS of (A.39) is bounded by a constant times

$$
n \sum_{l=L+1}^{\infty} \mathbb{E}\left\|N w_{l}\right\|^{2} \leq C n \sum_{l=L+1}^{\infty}\left(\mathbb{E}\left\|N w_{1 l}\right\|^{2}+\mathbb{E}\left\|N w_{2 l}\right\|^{2}+\mathbb{E}\left\|N w_{3 l}\right\|^{2}\right)
$$

by uncorrelatedness of $w_{l}$ due to Assumption 1, and the triangle and $c_{r}$ inequalities. The negligibility of $n \sum_{l=L+1}^{\infty} \mathbb{E}\left\|N w_{3 l}\right\|^{2}$ follows by the proof of (57) in Delgado and Robinson (2015), while the linearity in $\epsilon_{l}$ of $w_{2 l}$ implies that $n \sum_{l=L+1}^{\infty} \mathbb{E}\left\|N w_{2 l}\right\|^{2}$ is easily shown to be negligible as in Lemma A1 of Robinson and Thawornkaiwong (2012). It remains to prove that $n \sum_{l=L+1}^{\infty} \mathbb{E}\left\|N w_{1 l}\right\|^{2}$ is negligible. Notice that $\mathbb{E}\left\|N w_{1 l}\right\|^{2} \leq C n^{-2}\left\|\nu_{l l}^{\lambda}\right\|^{2}+C n^{-2} \mathbb{1}(l \geq 2) \sum_{k=1}^{l-1}\left\|\nu_{l k}^{\lambda}\right\|^{2} \leq C n^{-2} \sum_{k=1}^{l}\left\|\nu_{l k}^{\lambda}\right\|^{2}$, so that

$$
\begin{aligned}
& n \sum_{l=L+1}^{\infty} \mathbb{E}\left\|N w_{1 l}\right\|^{2} \leq C n^{-1} \sum_{l=L+1}^{\infty} \sum_{k=1}^{l}\left\|\nu_{l k}^{\lambda}\right\|^{2} \leq C n^{-1} \sum_{j=1}^{p} \sum_{l=L+1}^{\infty} c_{l}^{\prime} G_{j} \Xi^{-1} \sum_{k=1}^{l} c_{k} c_{k}^{\prime} \Xi^{-1} G_{j}^{\prime} c_{l} \\
\leq & C\|\Xi\| n^{-1} \sum_{j=1}^{p} \sum_{l=L+1}^{\infty} c_{l}^{\prime} G_{j} \Xi^{-1} \Xi^{-1} G_{j}^{\prime} c_{l} \leq C n^{-1} \sum_{j=1}^{p} \sum_{l=L+1}^{\infty} \sum_{r, s, t=1}^{n} c_{r l} c_{t l} p_{j r s} p_{j t s} \\
\leq & C n^{-1} \sum_{l=L+1}^{\infty} \sum_{r, t=1}^{n}\left(\sup _{\substack{j=1, \ldots, p \\
s=1, \ldots, n}}\left|p_{j r s}\right|\right)\left|c_{r l}^{*}\right|\left|c_{t l}^{*}\right|\left(\max _{j=1, \ldots, p} \sum_{s=1}^{n}\left|p_{j t s}\right|\right),
\end{aligned}
$$

where $p_{j r s}$ is the $(r, s)$-th element of $G_{j} \Xi^{-1}$. Note that

$$
\left|p_{j r s}\right| \leq \sum_{s=1}^{n}\left|p_{j r s}\right| \leq\left\|G_{j} \Xi^{-1}\right\|_{R}=\mathscr{O}_{p}(1)
$$


uniformly in $r, s, j$, by Assumptions 21 and 22. Thus (A.41) is

$$
\mathscr{O}_{p}\left(n^{-1} \sum_{r=1}^{n} \sum_{l=L+1}^{\infty}\left|c_{r l}^{*}\right| \sum_{t=1}^{n}\left|c_{t l}^{*}\right|\right)=\mathscr{O}_{p}\left(\sup _{r=1, \ldots, n} \sum_{l=L+1}^{\infty}\left|c_{r l}^{*}\right|\right),
$$

by Assumption 20. By the same assumption, there exists $L_{r n}$ such that $\sum_{l=L_{r n}+1}^{\infty}\left|c_{r l}^{*}\right| \leq \varepsilon_{n}$ for any decreasing sequence $\varepsilon_{n} \rightarrow 0$ as $n \rightarrow \infty$. Choosing $L=\max _{r=1, \ldots, n} L_{r n}$ in $d_{L}$, we deduce that (A.43) is $\mathscr{O}_{p}\left(\varepsilon_{n}\right)=o_{p}(1)$, proving (A.39). Thus we need only focus on $d_{L}$.

Define $D=n \mathbb{E}\left(N d_{L} d_{L}^{\prime} N\right)=n \sum_{l=1}^{L} N \mathbb{E}\left(w_{l} w_{l}^{\prime}\right) N$. In view of our subsequent calculations it is straightforward to show that $D \stackrel{p}{\rightarrow} \Delta$, implying that $D$ is positive definite for sufficiently large $n$ and assuring the existence of a square matrix, denoted $D^{\frac{1}{2}}$, such that $D^{\prime \frac{1}{2}} D^{\frac{1}{2}}=D$. For a $(p+k+q) \times 1$ constant vector $\alpha$ such that $\|\alpha\|=1$ define $s=n^{\frac{1}{2}} \alpha^{\prime} D^{-\frac{1}{2}} N d_{L}=n^{\frac{1}{2}} \sum_{l=1}^{L} \alpha^{\prime} D^{-\frac{1}{2}} N w_{l}$, which has zero mean and unit variance, and we seek to establish that

$$
s \stackrel{d}{\longrightarrow} \mathcal{N}(0,1) \text {, as } n \rightarrow \infty .
$$

From Scott (1973), (A.44) follows if

$$
\sum_{l=1}^{L} \mathbb{E}\left\|n^{\frac{1}{2}} D^{-\frac{1}{2}} N w_{l}\right\|^{4} \stackrel{p}{\longrightarrow} 0, \text { as } n \rightarrow \infty
$$

and

$$
n \sum_{l=1}^{L}\left[\mathbb{E}\left(N w_{l} w_{l}^{\prime} N \mid \epsilon_{k}, k<l\right)-\mathbb{E}\left(N w_{l} w_{l}^{\prime} N\right)\right] \stackrel{p}{\longrightarrow} 0, \text { as } n \rightarrow \infty .
$$

To prove (A.45) it is sufficient to show

$$
\sum_{l=1}^{L} \mathbb{E}\left\|n^{\frac{1}{2}} D^{-\frac{1}{2}} N w_{i l}\right\|^{4} \stackrel{p}{\longrightarrow} 0, \text { as } n \rightarrow \infty, i=1,2,3,
$$

by the triangle and $c_{r}$ inequalities.

For $i=3$ the proof of (A.47) is identical to the proof of claim (60) in Delgado and Robinson (2015). Writing $z_{l}^{\lambda}=A^{\prime} \Xi^{-1} c_{l}$ and $z_{l}^{\beta}=X^{\prime} \Xi^{-1} c_{l}$, for $i=2$ the left side of (A.47) is equal to a constant times $n^{-2} \sum_{l=1}^{L}\left(\left(z_{l}^{\prime \lambda} \epsilon_{l}, z_{l}^{\prime \beta} \epsilon_{l}, 0^{\prime}\right) D\left(z_{l}^{\prime \lambda} \epsilon_{l}, z_{l}^{\prime \beta} \epsilon_{l}, 0^{\prime}\right)^{\prime}\right)^{2}$, which is bounded by a constant times $n^{-2} \sum_{l=1}^{L}\left(\left\|z_{l}^{\lambda}\right\|^{4}+\left\|z_{l}^{\beta}\right\|^{4}\right)\|D\|^{2}$. The last factor in the latter is $\mathscr{O}_{p}(1)$ because $\|D\|^{2}$ converges in probability to $\|\Delta\|^{2}$, while

$$
\begin{aligned}
\sum_{l=1}^{L}\left\|z_{l}^{\lambda}\right\|^{4} & =\sum_{l=1}^{L}\left(z_{l}^{\prime \lambda} z_{l}^{\lambda}\right)^{2} \leq \sum_{l=1}^{\infty}\left(z_{l}^{\prime \lambda} z_{l}^{\lambda}\right)^{2}=\sum_{j=1}^{p} e_{j}^{\prime} \Xi^{-1} A \sum_{l=1}^{\infty} c_{l} c_{l}^{\prime} A^{\prime} \Xi^{-1} e_{j} \\
& =\sum_{j=1}^{p} e_{j}^{\prime} \Xi^{-1} A \Xi^{\prime} A^{\prime} \Xi^{-1} e_{j}=\mathscr{O}_{p}\left(\|A\|^{2}\left\|\Xi^{-1}\right\|^{2}\|\Xi\|\right) \\
& =\mathscr{O}_{p}\left(\|A\|^{2}\right)=\mathscr{O}_{p}\left(\max _{j=1, \ldots, p}\left\|G_{j}\right\|^{2}\|X\|^{2}\right)=\mathscr{O}_{p}(n),
\end{aligned}
$$


by Assumption 21 and (3.2). Similarly $\sum_{l=1}^{L}\left\|z_{l}^{\beta}\right\|^{4}=\mathscr{O}_{p}(n)$, so $\sum_{l=1}^{L} \mathbb{E}\left\|n^{\frac{1}{2}} D^{-\frac{1}{2}} N w_{2 l}\right\|^{4}=\mathscr{O}_{p}\left(n^{-1}\right)$ and (A.47) is proved for $i=2$. Finally consider (A.47) for $i=1$. Evaluating the expectation, using an $\ell_{p}$ norm inequality and employing techniques used earlier, it follows that

$$
\begin{aligned}
& \mathbb{E}\left\|D^{-\frac{1}{2}} N w_{1 l}\right\|^{4} \leq C n^{-4}\left\|\nu_{l l}^{\lambda}\right\|^{4}+C n^{-4} \mathbb{E}\left\|\sum_{k=1}^{l-1} \nu_{l k}^{\lambda} \epsilon_{k}\right\|^{4} \leq C n^{-4}\left\|\nu_{l l}^{\lambda}\right\|^{4}+C n^{-4}\left(\sum_{k=1}^{l-1}\left\|\nu_{l k}^{\lambda}\right\|^{2}\right)^{2} \\
\leq & C n^{-4}\left(\sum_{k=1}^{l}\left\|\nu_{l k}^{\lambda}\right\|^{2}\right)^{2} \leq C n^{-4}\left(\sum_{j=1}^{p} c_{l}^{\prime} G_{j} \Xi^{-1} \sum_{k=1}^{l} c_{k} c_{k}^{\prime} \Xi^{-1} G_{j}^{\prime} c_{l}\right)^{2}=\mathscr{O}_{p}\left(n^{-4}\left\|c_{l}\right\|^{4}\right),
\end{aligned}
$$

whence

$$
\begin{aligned}
\sum_{l=1}^{L} \mathbb{E}\left\|n^{\frac{1}{2}} D^{-\frac{1}{2}} N w_{2 l}\right\|^{4} & =\mathscr{O}_{p}\left(n^{-2} \sum_{l=1}^{L}\left\|c_{l}\right\|^{4}\right)=\mathscr{O}_{p}\left(n^{-2} \sum_{l=1}^{L}\left(\sum_{r=1}^{n} c_{r l}^{* 2}\right)^{2}\right) \\
& =\mathscr{O}_{p}\left(n^{-2} \sum_{l=1}^{L}\left(\sum_{r=1}^{n}\left|c_{r l}^{*}\right|\right)^{4}\right)=\mathscr{O}_{p}\left(n^{-2} \sum_{r=1}^{n}\left(\sum_{l=1}^{L}\left|c_{r l}^{*}\right|\right)\right)=\mathscr{O}_{p}\left(n^{-1}\right),
\end{aligned}
$$

by Assumption 20 and the $\ell_{p}$ norm inequality. Thus (A.47), and hence (A.45), is established.

To prove (A.46) it is sufficient to show

$$
n \sum_{l=1}^{L}\left[\mathbb{E}\left(N w_{i l} w_{j l}^{\prime} N \mid \epsilon_{k}, k<l\right)-\mathbb{E}\left(N w_{i l} w_{j l}^{\prime} N\right)\right] \stackrel{p}{\longrightarrow} 0, \text { as } n \rightarrow \infty, i, j=1,2,3, j \geq i
$$

Once again, for $i=j=3$ the proof is identical to that of (61) in Delgado and Robinson (2015) and for $i=j=2$ it follows easily as in the proof of a similar assertion in Theorem 2.2. Of the remaining cases the most complex to handle is $i=j=1$ in view of both its quadraticity in $u$ and involvement of the $G_{i}$, so we prove this first.

Notice that the only nonzero block of $\mathbb{E}\left(w_{1 l} w_{1 l}^{\prime} \mid \epsilon_{k}, k<l\right)$ equals

$$
\begin{aligned}
& 4 n^{-2} \sigma_{0}^{-4}\left\{\left(\mu_{4}-\sigma_{0}^{4}\right) \nu_{l l}^{\lambda} \nu_{l l}^{\prime \lambda}+\mu_{3} \mathbb{1}(l \geq 2) \sum_{k=1}^{l-1}\left(\nu_{l k}^{\lambda} \nu_{l l}^{\prime \lambda}+\nu_{l l}^{\lambda} \nu_{l k}^{\prime \lambda}\right) \epsilon_{k}\right\} \\
+ & 4 n^{-2} \sigma_{0}^{-2} \mathbb{1}(l \geq 2)\left(\sum_{k=1}^{l-1} \nu_{l k}^{\lambda} \epsilon_{k}\right)\left(\sum_{k=1}^{l-1} \nu_{l k}^{\lambda} \epsilon_{k}\right)^{\prime}
\end{aligned}
$$

with expectation $\mathbb{E}\left(w_{1 l} w_{1 l}^{\prime}\right)$ (we only consider the non-zero block) equal to $4 n^{-2} \sigma_{0}^{-4}\left(\mu_{4}-\sigma_{0}^{4}\right) \nu_{l l}^{\lambda} \nu_{l l}^{\prime \lambda}+$ $4 n^{-2} \mathbb{1}(l \geq 2) \sum_{k=1}^{l-1} \nu_{l k}^{\lambda} \nu_{l k}^{\prime \lambda}$, so that the Frobenius norm of (A.48) for $i=j=1$ is bounded by a constant times

$$
n^{-1}\left\|\sum_{l=2}^{L} \sum_{k=1}^{l-1}\left(\nu_{l k}^{\lambda} \nu_{l l}^{\prime \lambda}+\nu_{l l}^{\lambda} \nu_{l k}^{\prime \lambda}\right) \epsilon_{k}\right\|_{F}+n^{-1}\left\|\sum_{l=2}^{L}\left(\left(\sum_{k=1}^{l-1} \nu_{l k}^{\lambda} \epsilon_{k}\right)\left(\sum_{k=1}^{l-1} \nu_{l k}^{\lambda} \epsilon_{k}\right)^{\prime}-\sigma_{0}^{2} \sum_{k=1}^{l-1} \nu_{l k}^{\lambda} \nu_{l k}^{\prime \lambda}\right)\right\|_{F} .
$$

By transforming the range of summation, the square of the first term in (A.49) has expectation 
bounded by

$$
C n^{-2} \mathbb{E}\left\|\sum_{k=1}^{L-1}\left(\sum_{l=k+1}^{L}\left(\nu_{l k}^{\lambda} \nu_{l l}^{\prime \lambda}+\nu_{l l}^{\lambda} \nu_{l k}^{\prime \lambda}\right)\right) \epsilon_{k}\right\|_{F}^{2} \leq C n^{-2} \sum_{k=1}^{L-1}\left\|\sum_{l=k+1}^{L}\left(\nu_{l k}^{\lambda} \nu_{l l}^{\prime \lambda}+\nu_{l l}^{\lambda} \nu_{l k}^{\prime \lambda}\right)\right\|_{F}^{2},
$$

where the factor in the norm on the RHS of (A.50) is

$$
\begin{aligned}
& \sum_{i, j=1}^{p} \sum_{l, m=k+1}^{L} c_{l}^{\prime} G_{i} \Xi^{-1} c_{l} c_{l}^{\prime} G_{j} \Xi^{-1} c_{k} c_{m}^{\prime} G_{i} \Xi^{-1} c_{m} c_{m}^{\prime} G_{j} \Xi^{-1} c_{k} \\
\leq & \sum_{l, m=k+1}^{L}\left|\sum_{i=1}^{p} c_{l}^{\prime} G_{i} \Xi^{-1} c_{l} c_{m}^{\prime} G_{i} \Xi^{-1} c_{m}\right| \sum_{j=1}^{p}\left|c_{l}^{\prime} G_{j} \Xi^{-1} c_{k}\right|\left|c_{m}^{\prime} G_{j} \Xi^{-1} c_{k}\right| \\
\leq & C \sum_{l, m=k+1}^{L} \max _{i=1, \ldots, p} \sum_{r, s, u, t=1}^{n}\left|c_{r l}\right|\left|p_{i r s}\right|\left|c_{s m}\right|\left|c_{u l}\right|\left|p_{i u t}\right|\left|c_{t m}\right| \max _{j=1, \ldots, p}\left|c_{l}^{\prime} G_{j} \Xi^{-1} c_{k}\right|\left|c_{m}^{\prime} G_{j} \Xi^{-1} c_{k}\right| \\
\leq & C\left(\sup _{i, r, s}\left|p_{i r s}\right|\right)^{2}\left(\sup _{l \geq 1}^{n} \sum_{r=1}^{n}\left|c_{r l}^{*}\right|\right)^{4} \sum_{l, m=k+1}^{L} \max _{j=1, \ldots, p}\left|c_{l}^{\prime} G_{j} \Xi^{-1} c_{k}\right|\left|c_{m}^{\prime} G_{j} \Xi^{-1} c_{k}\right| \\
= & \mathscr{O}_{p}\left(\max _{j=1, \ldots, p}\left(\sum_{l=k+1}^{L}\left|c_{k}^{\prime} G_{j} \Xi^{-1} c_{l}\right|\right)^{2}\right)=\mathscr{O}_{p}\left(\max _{j=1, \ldots, p}\left(\sum_{l=k+1}^{L} \sum_{r, s=1}^{n}\left|c_{r k}^{*}\right|\left|p_{j r s}\right|\left|c_{s l}^{*}\right|\right)^{2}\right),
\end{aligned}
$$

where we used Assumptions 20 and (A.42). Now Assumptions 20, 21, 22 and (A.42) imply that, uniformly in $j, \sum_{l=k+1}^{L} \sum_{r, s=1}^{n}\left|c_{r k}^{*}\right|\left|p_{j r s}\right|\left|c_{s l}^{*}\right|=\sum_{r=1}^{n}\left|c_{r k}^{*}\right| \sum_{s=1}^{n}\left|p_{j r s}\right| \sum_{l=k+1}^{L}\left|c_{s l}^{*}\right|=\mathscr{O}_{p}\left(\sum_{r=1}^{n}\left|c_{r k}^{*}\right|\right)$, so (A.50) is $\mathscr{O}_{p}\left(n^{-2} \sup _{k \geq 1}\left(\sum_{r=1}^{n}\left|c_{r k}^{*}\right|\right)\left(\sum_{r=1}^{n}\left(\sum_{k=1}^{L-1}\left|c_{r k}^{*}\right|\right)\right)\right)$. By Assumption 20 the latter is $\mathscr{O}_{p}\left(n^{-1}\right)$ and therefore the first term in (A.49) is $\mathscr{O}_{p}\left(n^{-\frac{1}{2}}\right)$.

Once again transforming the range of summation, we can rewrite the square of the second term in (A.49) as

$$
n^{-2}\left\|\sum_{k=1}^{L-1} \sum_{l=k+1}^{L} \nu_{l k}^{\lambda} \nu_{l k}^{\prime \lambda}\left(\epsilon_{k}^{2}-\sigma_{0}^{2}\right)+\sum_{k=1}^{L-1} \sum_{m=1}^{k-1}\left(\sum_{l=k+1}^{L}\left(\nu_{l k}^{\lambda} \nu_{l m}^{\prime \lambda}+\nu_{l m}^{\lambda} \nu_{l k}^{\prime \lambda}\right)\right) \epsilon_{k} \epsilon_{m}\right\|_{F}^{2} .
$$

A calculation along the lines of the proof of assertion (64) in Delgado and Robinson (2015) establishes that (A.51) is $\mathscr{O}_{p}\left(\varkappa_{1}+\varkappa_{2}\right)$, where

$$
\begin{aligned}
\varkappa_{1} & =n^{-2} \sum_{k=1}^{L-1}\left(\sum_{j=1}^{p} \sum_{l=k+1}^{L} \sum_{r, s=1}^{n}\left|c_{r l}^{*}\right|\left|p_{j r s}\right|\left|c_{s k}^{*}\right|\right)^{2} \\
\varkappa_{2} & =n^{-2} \sum_{j, k, l, m=1}^{L} \sum_{i_{1}, i_{2}=1}^{p} \sum_{r, s=1}^{n}\left|c_{r k}^{*}\right|\left|p_{i_{1} r s}\right|\left|c_{s j}^{*}\right| \sum_{r, s=1}^{n}\left|c_{r k}^{*}\right|\left|p_{i_{2} r s}\right|\left|c_{s m}^{*}\right| \\
& \times \sum_{r, s=1}^{n}\left|c_{r l}^{*}\right|\left|p_{i_{1} r s}\right|\left|c_{s j}^{*}\right| \sum_{r, s=1}^{n}\left|c_{r l}^{*}\right|\left|p_{i_{1} r s}\right|\left|c_{s m}^{*}\right|
\end{aligned}
$$


Now by (A.42), Assumptions 20, 21, 22 and elementary inequalities $\varkappa_{2}$ is

$$
\begin{aligned}
& \mathscr{O}_{p}\left(n^{-2} \max _{i_{1}, i_{2}=1, \ldots, p} \sum_{j, k, l=1}^{L}\left(\sum_{r, s=1}^{n}\left|c_{r k}^{*}\right|\left|p_{i_{1} r s}\right|\left|c_{s j}^{*}\right|\right)\left(\sum_{r, s=1}^{n}\left|c_{r k}^{*}\right|\left|p_{i_{2} r s}\right| \sum_{m=1}^{L}\left|c_{s m}^{*}\right|\right)\right. \\
\times & \left.\sum_{r, s=1}^{n}\left|c_{r l}^{*}\right|\left|p_{i_{1} r s}\right|\left|c_{s j}^{*}\right| \sum_{r=1}^{n}\left|c_{r l}^{*}\right| \sum_{s=1}^{n}\left|c_{s m}^{*}\right|\right) \\
= & \mathscr{O}_{p}\left(n^{-2} \max _{i_{1}=1, \ldots, p} \sum_{j, k=1}^{L}\left(\sum_{r, s=1}^{n}\left|c_{r k}^{*}\right|\left|p_{i_{1} r s}\right|\left|c_{s j}^{*}\right|\right) \sum_{r=1}^{n}\left|c_{r k}^{*}\right|\left(\sum_{r, s=1}^{n} \sum_{l=1}^{L}\left|c_{r l}^{*}\right|\left|p_{i_{1} r s}\right|\left|c_{s j}^{*}\right|\right)\right) \\
= & \mathscr{O}_{p}\left(n^{-2} \max _{i_{1}=1, \ldots, p} \sum_{r, s=1}^{n}\left(\sum_{k=1}^{L}\left|c_{r k}^{*}\right|\right)\left|p_{i_{1} r s}\right|\left(\sum_{j=1}^{L}\left|c_{s j}^{*}\right|\right) \sum_{s=1}^{n}\left(\sum_{r=1}^{n}\left|p_{i_{1} r s}\right|\right)\left|c_{s j}^{*}\right|\right) \\
= & \mathscr{O}_{p}\left(n^{-1} \max _{i_{1}=1, \ldots, p}\left\|\Xi^{-1} G_{i_{1}}^{\prime}\right\| \|_{R}\right)=\mathscr{O}_{p}\left(n^{-1}\right),
\end{aligned}
$$

while a similar use of the conditions of the theorem implies that $\varkappa_{1}$ is

$$
\begin{aligned}
& \mathscr{O}_{p}\left(n^{-2} \sum_{k=1}^{L-1}\left(\max _{j=1, \ldots, p} \sum_{r, s=1}^{n}\left|p_{j r s}\right|\left|c_{s k}^{*}\right|\right)^{4}\right)=\mathscr{O}_{p}\left(n^{-2} \sum_{k=1}^{L-1}\left(\sum_{s=1}^{n}\left|c_{s k}^{*}\right|\right)^{4}\right) \\
= & \mathscr{O}_{p}\left(n^{-2} \sum_{s=1}^{n} \sum_{k=1}^{L-1}\left|c_{s k}^{*}\right|\left(\sum_{s=1}^{n}\left|c_{s k}^{*}\right|\right)^{3}\right)=\mathscr{O}_{p}\left(n^{-2} \sum_{s=1}^{n} \sum_{k=1}^{L-1}\left|c_{s k}^{*}\right|\right)=\mathscr{O}_{p}\left(n^{-1}\right),
\end{aligned}
$$

proving (A.48) for $i=j=1$, as desired. For the remaining combinations of $i$ and $j$ we discuss the proof briefly to avoid repetition.

When $i=1, j=2$, the only nonzero blocks of $\mathbb{E}\left(w_{1 l} w_{2 l}^{\prime} \mid \epsilon_{k}, k<l\right)$ equal $4 n^{-2} \sigma_{0}^{-4} \mu_{3} \nu_{l l}^{\lambda} c_{l}^{\prime} \Xi^{-1} A+$ $8 n^{-2} \sigma_{0}^{-2} \mathbb{1}(l \geq 2) \sum_{k=1}^{l-1} \nu_{l k}^{\lambda} c_{l}^{\prime} \Xi^{-1} A \epsilon_{k}$ and $4 n^{-2} \sigma_{0}^{-4} \mu_{3} \nu_{l l}^{\lambda} c_{l}^{\prime} \Xi^{-1} X+8 n^{-2} \sigma_{0}^{-2} \mathbb{1}(l \geq 2) \sum_{k=1}^{l-1} \nu_{l k}^{\lambda} c_{l}^{\prime} \Xi^{-1} X \epsilon_{k}$ with expectations respectively equal to $4 n^{-2} \sigma_{0}^{-4} \mu_{3} \nu_{l l}^{\lambda} c_{l}^{\prime} \Xi^{-1} A$ and $4 n^{-2} \sigma_{0}^{-4} \mu_{3} \nu_{l l}^{\lambda} c_{l}^{\prime} \Xi^{-1} X$. Thus the Frobenius norm of (A.48) for $i=1, j=2$ is bounded by a constant times

$$
n^{-1}\left\|\sum_{l=2}^{L} \sum_{k=1}^{l-1} \nu_{l k}^{\lambda} c_{l}^{\prime} \Xi^{-1} A \epsilon_{k}\right\|_{F}+n^{-1}\left\|\sum_{l=2}^{L} \sum_{k=1}^{l-1} \nu_{l k}^{\lambda} c_{l}^{\prime} \Xi^{-1} X \epsilon_{k}\right\|_{F} .
$$

Writing $A_{j}=G_{j} X \beta_{0}, j=1, \ldots, p$, and proceeding as in the earlier proof for $i=j=1$ we deduce that the expectation of the square of the first term in (A.54) is bounded by

$$
\begin{aligned}
C n^{-2} \sum_{k=1}^{L-1}\left\|\sum_{l=k+1}^{L} \nu_{l k}^{\lambda} c_{l}^{\prime} \Xi^{-1} A\right\|_{F}^{2}= & C n^{-2} \sum_{k=1}^{L-1} \sum_{i, j=1}^{p}\left\{c_{k}^{\prime} \Xi^{-1} G_{i}^{\prime}\left(\sum_{l=k+1}^{L} c_{l} c_{l}^{\prime}\right) \Xi^{-1} A_{i}\right. \\
& \left.\times A_{j}^{\prime} \Xi^{-1}\left(\sum_{m=k+1}^{L} c_{m} c_{m}^{\prime}\right) G_{j} \Xi^{-1} c_{k}\right\} \\
\leq & C n^{-2} \sum_{k=1}^{L-1} \sum_{i, j=1}^{p}\left\{\left(c_{k}^{\prime} \Xi^{-1} G_{i}^{\prime} G_{i} \Xi^{-1} c_{k} c_{k}^{\prime} \Xi^{-1} G_{j}^{\prime} G_{j} \Xi^{-1} c_{k}\right)^{\frac{1}{2}}\right. \\
& \left.\times\left(A_{i}^{\prime} \Xi^{-1} \Xi^{-1} A_{i} A_{j}^{\prime} \Xi^{-1} \Xi^{-1} A_{j}\right)^{\frac{1}{2}}\right\}
\end{aligned}
$$




$$
\leq C n^{-2} \max _{j=1, \ldots, p}\left(A_{j}^{\prime} \Xi^{-2} A_{j}\right) \sum_{k=1}^{L-1}\left(c_{k}^{\prime} \Xi^{-1} G_{j}^{\prime} G_{j} \Xi^{-1} c_{k}\right)
$$

where we use the inequality $x^{\prime} Z y \leq\|x\|\|Z\|\|y\|$ and the fact that $\left\|\sum_{l=k+1}^{L} c_{l} c_{l}^{\prime}\right\| \leq\|\Xi\|=\mathscr{O}(1)$. The first factor in parentheses in (A.55) is $\mathscr{O}_{p}\left(\left\|A_{j}\right\|_{R}^{2}\left\|\Xi^{-1}\right\|_{R}^{2}\right)=\mathscr{O}_{p}\left(\left\|G_{j}\right\|_{R}^{2}\|X\|_{R}^{2}\right)=\mathscr{O}_{p}(1)$, uniformly in $j$ by Assumptions 21, 22 and (3.2). The sum in (A.55) is bounded by

$$
\begin{aligned}
& \sum_{k=1}^{\infty} \sum_{r, s=1}^{n} c_{r k} c_{s k}\left(\sum_{t=1}^{n} p_{j t r} p_{j t s}\right)=\operatorname{tr}\left(\Xi^{-1} G_{j}^{\prime} G_{j} \Xi^{-1}\right)=\operatorname{tr}\left(\Xi^{-\frac{1}{2}} G_{j}^{\prime} G_{j} \Xi^{\prime-\frac{1}{2}}\right)=\left\|\Xi^{-\frac{1}{2}} G_{j}^{\prime}\right\|_{F}^{2} \\
= & \mathscr{O}_{p}\left(\left\|\Xi^{-\frac{1}{2}}\right\|_{F}^{2}\right)=\operatorname{tr}\left(\Xi^{-1}\right)=\mathscr{O}_{p}(n),
\end{aligned}
$$

uniformly in $j$ by (A.23) and Assumption 12. The first term in (A.54) is thus established to be of order $\mathscr{O}_{p}\left(n^{-\frac{1}{2}}\right)$, which is negligible. The second term in (A.54) is handled in an identical fashion.

When $i=1, j=3$, we consider the product of two zero mean blocks that are quadratic in $\epsilon_{l}$, as in the $i=j=1$ case, and the desired conclusion follows on proceeding as for that case. Finally the case $i=2, j=3$ involves a block that is the product of a linear and a quadratic term in $\epsilon$, so it will also yield a term like (A.54) except with $\nu_{l k}^{\lambda}$ replaced with $\nu_{l k}^{\tau}$, whence the details follow as above and are omitted. Thus (A.37) is established.

For the next step of the proof we will introduce the second derivative matrix of (4.1) at any admissible point $\left(\varrho^{\prime}, \sigma^{2}\right)^{\prime}$ in the parameter space. Write $\mathcal{R}^{y}(\theta)=y-R \lambda-X \beta=S(\lambda) y-X \beta$, and introduce the following matrices: $F_{8}(\varrho)$ of dimension $p \times q$ and $j$-th column $R^{\prime} \Xi(\tau)^{-1} \Xi_{j}(\tau) \Xi(\tau)^{-1} \mathcal{R}^{y}(\theta), F_{9}(\varrho)$ of dimension $k \times q$ with $j$-th column $X^{\prime} \Xi(\tau)^{-1} \Xi_{j}(\tau) \Xi(\tau)^{-1} \mathcal{R}^{y}(\theta)$, and finally $F_{10}(\varrho)$ and $P_{\Xi 2}(\tau)$ both of dimension $q \times q$ with $(i, j)$-th elements $\mathcal{R}^{\prime y}(\theta) \Xi(\tau)^{-1}\left\{2 \Xi_{i}(\tau) \Xi(\tau)^{-1} \Xi_{j}(\tau)-\Xi_{i j}(\tau)\right\} \Xi(\tau)^{-1} \mathcal{R}^{y}(\theta)$ and $\operatorname{tr}\left(\Xi(\tau)^{-1}\left\{\Xi_{i j}(\tau)-\Xi_{i}(\tau) \Xi(\tau)^{-1} \Xi_{j}(\tau)\right\}\right)$, respectively. Then

$$
H\left(\varrho, \sigma^{2}\right)=2 \sigma^{-2} n^{-1}\left[\begin{array}{ccc}
\sigma^{2} P_{G 1}(\lambda)+R^{\prime} \Xi(\tau)^{-1} R & R^{\prime} \Xi(\tau)^{-1} X & F_{8}(\varrho) \\
* & X^{\prime} \Xi(\tau)^{-1} X & F_{9}(\varrho) \\
* & * & 2^{-1}\left(F_{10}(\varrho)+\sigma^{2} P_{\Xi 2}(\tau)\right)
\end{array}\right],
$$

so that

$$
H=2 \sigma_{0}^{-2} n^{-1}\left[\begin{array}{ccc}
\sigma_{0}^{2} P_{G 1}+R^{\prime} \Xi^{-1} R & R^{\prime} \Xi^{-1} X & F_{8} \\
* & X^{\prime} \Xi^{-1} X & F_{9} \\
* & * & 2^{-1}\left(F_{10}+\sigma^{2} P_{\Xi 2}\right)
\end{array}\right]
$$

whence, noting that $\mathcal{R}^{y}=u$, the expression in (4.11) follows easily. By the mean value theorem $(\mathrm{MVT})$, we have $0=d+H\left(\bar{\varrho}, \check{\sigma}^{2}\right)\left(\check{\varrho}-\varrho_{0}\right)$, where $H\left(\bar{\varrho}, \check{\sigma}^{2}\right)$ is obtained from $H\left(\varrho, \sigma^{2}\right)$ by evaluating each row at possibly different $\bar{\varrho}$ satisfying $\left\|\bar{\varrho}-\varrho_{0}\right\| \leq\left\|\varrho-\varrho_{0}\right\|$. Writing $0=N d+$ $N H\left(\bar{\varrho}, \check{\sigma}^{2}\right) N N^{-1}\left(\check{\varrho}-\varrho_{0}\right)$, we obtain $n^{\frac{1}{2}} N^{-1}\left(\check{\varrho}-\varrho_{0}\right)=-\left(N H\left(\bar{\varrho}, \check{\sigma}^{2}\right) N\right)^{-1} n^{\frac{1}{2}} N d$, indicating that the theorem is proved if we also show that

$$
N\left(H\left(\bar{\varrho}, \check{\sigma}^{2}\right)-H\right) N \stackrel{p}{\longrightarrow} 0, N(H-\Sigma) N \stackrel{p}{\longrightarrow} 0, \text { as } n \rightarrow \infty,
$$

because we have shown (A.37). The first part above uses $\bar{\varrho}-\varrho_{0} \stackrel{p}{\rightarrow} 0$ and $\check{\sigma}^{2}-\sigma_{0}^{2} \stackrel{p}{\rightarrow} 0$ together with the regularity conditions imposed on $\Xi(\tau)$ and $\Xi(\tau)^{-1}$, and the second follows using law of large numbers 
arguments. The calculations are handled in the same fashion as in the existing SAR literature, cf. Lee (2004), Delgado and Robinson (2015) and Gupta and Robinson (2018), so we omit the details except for the proof of

$$
n^{-1}\left(P_{G 1}(\bar{\lambda})-P_{G 1}\right) \stackrel{p}{\longrightarrow} 0,
$$

which is the most relevant and novel in this paper given our focus on stochastic $W_{j}$. The proof of (A.59) commences as in Lee (2004) and Gupta and Robinson (2018), by using the MVT to write the $(i, j)$-th element of the left side as $n^{-1} \operatorname{tr}\left(G_{i}(\bar{\lambda}) G_{j}(\bar{\lambda})\right)-n^{-1} \operatorname{tr}\left(G_{i} G_{j}\right)=n^{-1} \overline{\bar{g}}^{\prime}\left(\bar{\lambda}-\lambda_{0}\right)$, where $\overline{\bar{g}}$ is a $p \times 1$ vector with $k$-th element $\operatorname{tr}\left(G_{i}(\overline{\bar{\lambda}}) G_{k}(\overline{\bar{\lambda}}) G_{j}(\overline{\bar{\lambda}})+G_{k}(\overline{\bar{\lambda}}) G_{i}(\overline{\bar{\lambda}}) G_{j}(\overline{\bar{\lambda}})\right)$ and $\overline{\bar{\lambda}}$ satisfies $\left\|\overline{\bar{\lambda}}-\lambda_{0}\right\| \leq\left\|\bar{\lambda}-\lambda_{0}\right\| \leq\left\|\check{\lambda}-\lambda_{0}\right\|$. Because $\left\|\bar{\lambda}-\lambda_{0}\right\| \stackrel{p}{\rightarrow} 0$, (A.59) is proved if $n^{-1} \overline{\bar{g}}=\mathscr{O}_{p}(1)$. Since Lemma B.6 implies that each element of $\overline{\bar{g}}$ is $\mathscr{O}_{p}\left(n / h_{W}\right)$, the latter claim is true. The proof of the theorem is now completed.

\section{B Lemmas}

Lemma B.1. Under the conditions of Theorem 3.1, the expectation of an absolute typical element of $G_{j}^{\prime} G_{j}$ is $\mathscr{O}\left(\pi_{n}^{2}\right)$, uniformly in $j$.

Proof. For $r, s=1, \ldots, n$, a typical absolute element of $G_{j}^{\prime} G_{j}$ is $\left|g_{r, j}^{\prime} G_{j} e_{s}\right|=\left|e_{s}^{\prime} G_{j}^{\prime} g_{r, j}\right|$, where $g_{r, j}^{\prime}$ is the $r$-th row of $G_{j}^{\prime}$. Using Hölder's inequality as before, this has expectation bounded by

$$
\left(\mathbb{E}\left\|g_{r, j}\right\|_{R}^{\zeta_{5}}\right)^{\frac{1}{\zeta_{5}}}\left(\mathbb{E}\left\|G_{j}^{\prime}\right\|_{R}^{\zeta_{6}}\right)^{\frac{1}{\zeta_{6}}} \leq\left(\mathbb{E}\left\|g_{r, j}\right\|_{R}^{\zeta_{5}}\right)^{\frac{1}{\zeta_{5}}}\left(\mathbb{E}\left(\left\|W_{j}^{\prime}\right\|_{R}^{\zeta_{6}}\left\|S^{\prime-1}\right\|_{R}^{\zeta_{6}}\right)\right)^{\frac{1}{\zeta_{6}}}
$$

Consider the first factor on the RHS of (B.1). $g_{r, j}$ has elements $w_{s, j}^{\prime} S^{-1} e_{r}=e_{r}^{\prime} S^{\prime-1} w_{s, j}$, where $w_{s, j}^{\prime}$ is the $s$-th row of $W_{j}, s=1, \ldots, n$, so this factor is

$$
\begin{aligned}
\left(\mathbb{E}\left(\max _{1 \leq s \leq n}\left|w_{s, j}^{\prime} S^{-1} e_{r}\right|^{\zeta_{5}}\right)\right)^{\frac{1}{\zeta_{5}}} & \leq\left(\mathbb{E}\left(\max _{1 \leq s \leq n}\left\|w_{s, j}\right\|_{R}^{\zeta_{5}}\left\|S^{\prime-1}\right\|_{R}^{\zeta_{5}}\right)\right)^{\frac{1}{\zeta_{5}}} \\
& \leq\left(\mathbb{E}\left(\max _{1 \leq s \leq n}\left\|w_{s, j}\right\|_{R}^{\zeta_{5} \zeta_{7}}\right)\right)^{\frac{1}{\zeta_{5} \zeta_{7}}}\left(\mathbb{E}\left(\left\|S^{\prime-1}\right\|_{R}^{\zeta_{5} \zeta_{8}}\right)\right)^{\frac{1}{\zeta_{5} \zeta_{8}}} \\
& =\left(\mathbb{E}\left(\max _{1 \leq r, s \leq n}\left|w_{r s, j}\right|^{\zeta_{5} \zeta_{7}}\right)\right)^{\frac{1}{\zeta_{5} \zeta_{7}}}\left(\mathbb{E}\left(\left\|S^{\prime-1}\right\|_{R}^{\zeta_{5} \zeta_{8}}\right)\right)^{\frac{1}{\zeta_{5} \zeta_{8}}} \\
& =\mathscr{O}\left(h^{-1}\left(\mathbb{E}\left(\left\|S^{\prime-1}\right\|_{R}^{\zeta_{5} \zeta_{8}}\right)\right)^{\frac{1}{\zeta_{5} \zeta_{8}}}\right)
\end{aligned}
$$

by the Hölder inequality and Assumption 7. The second factor on the RHS of (B.1) is bounded by

$$
\left(\max _{1 \leq j \leq p} \mathbb{E}\left\|W_{j}^{\prime}\right\|_{R}^{\zeta_{6} \zeta_{9}}\right)^{\frac{1}{\zeta_{6} \zeta_{9}}}\left(\mathbb{E}\left\|S^{\prime-1}\right\|_{R}^{\zeta_{6} \zeta_{10}}\right)^{\frac{1}{\zeta_{6} \zeta_{10}}}
$$

by another application of Hölder's inequality, whence the claim follows from (B.1), (B.2), (B.3) and the definition of $\pi_{n}$.

Lemma B.2. Under the conditions of Theorem 3.2, the expectation of the absolute product of two typical elements of $G_{j}$ is $\mathscr{O}\left(\left(\mathbb{E}\left\|S^{\prime-1}\right\|_{R}^{2 \zeta_{12}}\right)^{\frac{1}{\zeta_{12}}} h^{-2}\right)$, uniformly in $j$. 
Proof. For $p, q, r, s=1, \ldots, n$, the expectation of the absolute product of typical elements of $G_{j}$ is

$$
\mathbb{E}\left|w_{r, j}^{\prime} S^{-1} e_{s} w_{p, j}^{\prime} S^{-1} e_{q}\right| \leq \mathbb{E}\left(\max _{1 \leq r \leq n}\left\|w_{r, j}\right\|_{R}^{2}\left\|S^{\prime-1}\right\|_{R}^{2}\right)
$$

which is bounded by $\left(\mathbb{E}\left(\max _{1 \leq r \leq n}\left\|w_{r, j}\right\|_{R}^{2 \zeta_{11}}\right)\right)^{\frac{1}{\zeta_{11}}}\left(\mathbb{E}\left\|S^{\prime-1}\right\|_{R}^{2 \zeta_{12}}\right)^{\frac{1}{\zeta_{12}}}$, whence the result follows by Assumption 7 because $\left\|w_{r, j}\right\|_{R}=\max _{1 \leq s \leq n}\left|w_{r s, j}\right|$.

Lemma B.3. Under the conditions of Theorem 3.1, $n^{-1}[B, 0]^{\prime} u=\mathscr{O}_{p}\left(\pi_{n}\right)$.

Proof. First note that $\mathbb{E}\|u\|^{2}=\sigma^{2} \sum_{r=1}^{n} \sum_{l=1}^{\infty} c_{r l}^{2}=\mathscr{O}(n)$, by $(2.4)$, so $\left\|n^{-1}[B, 0]^{\prime} u\right\|=\mathscr{O}_{p}\left(n^{-\frac{1}{2}}\|B\|\right)$ by Markov's inequality. Next $\mathbb{E}\|B\|^{2} \leq \mathbb{E}\left(\operatorname{tr} B^{\prime} B\right)=\mathscr{O}\left(\max _{1 \leq j \leq p} \mathbb{E}\left(u^{\prime} G_{j}^{\prime} G_{j} u\right)\right)$, the RHS being

$$
\mathscr{O}_{p}\left(\pi_{n}^{2} \sum_{r, s=1}^{n} \sum_{j, l=1}^{\infty} c_{r j} c_{s l} \mathbb{E}\left(\epsilon_{j} \epsilon_{l}\right)\right)=\mathscr{O}_{p}\left(\pi_{n}^{2} \sum_{r, s=1}^{n} \sum_{l=1}^{\infty}\left(c_{r l}^{2}+c_{s l}^{2}\right)\right)=\mathscr{O}_{p}\left(\pi_{n}^{2} \sum_{r=1}^{n} \sum_{l=1}^{\infty} c_{r l}^{2}\right)=\mathscr{O}_{p}\left(n \pi_{n}^{2}\right),
$$

by Lemma B.1, the inequality $|a b| \leq\left(a^{2}+b^{2}\right) / 2$ for real numbers $a, b$ and Assumption 1 . The claim follows by Markov's inequality,

Lemma B.4. Under the conditions of Theorem 3.2, $n^{-1}[B, 0]^{\prime} u=\mathscr{O}_{p}\left(\left(\mathbb{E}\left\|S^{\prime-1}\right\|_{R}^{2 \zeta_{12}}\right)^{\frac{1}{2 \zeta_{12}}} h^{-1}\right)$. Proof. Write $g_{r s, j}$ for a typical element of $G_{j}, r, s=1, \ldots, n$. It is sufficient to evaluate

$$
\mathbb{E}\left(n^{-1} u^{\prime} G_{j} u\right)^{2}=n^{-2} \sum_{r, s, t, v=1}^{n} \mathbb{E}\left(u_{r} u_{s} u_{t} u_{v}\right) \mathbb{E}\left(g_{r s, j} g_{t v, j}\right),
$$

with $j=1, \ldots, p$, and then use Markov's inequality. By Assumption 1 and Lemma B.2 the RHS of (B.5) is

$$
\begin{aligned}
& n^{-2} \sum_{r, s, t, v=1}^{n} \sum_{j, k, l, m=1}^{\infty} c_{r j} c_{s k} c_{t l} c_{v m} \mathbb{E}\left(\epsilon_{j} \epsilon_{k} \epsilon_{l} \epsilon_{m}\right) \mathbb{E}\left(g_{r s, j} g_{t v, j}\right) \\
= & \mathscr{O}\left(n ^ { - 2 } ( \mathbb { E } \| S ^ { \prime - 1 } \| _ { R } ^ { 2 \zeta _ { 1 2 } } ) ^ { \frac { 1 } { \zeta _ { 1 2 } } } h ^ { - 2 } \left[\sum_{r, s, t, v=1}^{n} \sum_{j=1}^{\infty} \mathbb{E}\left(\epsilon_{j}^{4}\right) c_{r j} c_{s j} c_{t j} c_{v j}+\right.\right. \\
+ & \left.\left.\sum_{r, s, t, v=1}^{n} \sum_{j, k=1}^{\infty}\left(c_{r j} c_{s j} c_{t k} c_{v k}+c_{r j} c_{s k} c_{t j} c_{v k}+c_{r j} c_{s k} c_{t k} c_{v j}\right)\right]\right) .
\end{aligned}
$$

By Assumption 9 and the $\ell_{p}$ norm inequality, the first sum inside square brackets in (B.6) is bounded in absolute value by a constant times

$$
\begin{aligned}
\sum_{r, s, t, v=1}^{n} \sum_{j=1}^{\infty}\left|c_{r j} c_{s j} c_{t j} c_{v j}\right| & \leq C \sum_{r, s, t, v=1}^{n} \sum_{j=1}^{\infty}\left(c_{r j}^{2} c_{s j}^{2}+c_{t j}^{2} c_{v j}^{2}\right) \\
& \leq C \sum_{r, s, t, v=1}^{n} \sum_{j=1}^{\infty}\left(c_{r j}^{4}+c_{s j}^{4}+c_{t j}^{4}+c_{v j}^{4}\right)
\end{aligned}
$$




$$
\leq C \sum_{r=1}^{n} \sum_{j=1}^{\infty} c_{r j}^{4} \leq C \sum_{r=1}^{n}\left(\sum_{j=1}^{\infty} c_{r j}^{2}\right)^{2} \leq C n
$$

Now consider the first product inside parentheses in the second sum inside square brackets in (B.6). By similar techniques this is bounded in absolute value by

$$
\begin{aligned}
\sum_{r, s, t, v=1}^{n} \sum_{j, k=1}^{\infty}\left|c_{r j} c_{s j}\right|\left|c_{t k} c_{v k}\right|=\left(\sum_{r, s=1}^{n} \sum_{j=1}^{\infty}\left|c_{r j} c_{s j}\right|\right)^{2} & \leq C\left(\sum_{r, s=1}^{n} \sum_{j=1}^{\infty}\left(c_{r j}^{2}+c_{s j}^{2}\right)\right)^{2} \\
& \leq C\left(\sum_{r=1}^{n}\left(\sum_{j=1}^{\infty} c_{r j}^{2}\right)\right)^{2} \leq C n^{2} .
\end{aligned}
$$

The remaining two products inside parentheses in the second sum inside square brackets in (B.6) are similarly shown to be $\mathscr{O}\left(n^{2}\right)$. We have established that the term inside square brackets in (B.6) is $\mathscr{O}\left(n^{2}\right)$, whence the claim follows.

Lemma B.5. Under the conditions of Theorem 4.2, $\left\|S(\lambda)^{-1}\right\|_{R}=\mathscr{O}_{p}(1)$ and $\left\|S(\lambda)^{\prime-1}\right\|_{R}=\mathscr{O}_{p}(1)$ in a closed neighbourhood of $\lambda_{0}$, denoted $B\left(\lambda_{0}\right)$.

Proof. We prove the claim for $\left\|S(\lambda)^{-1}\right\|_{R}$ only, the proof for the transpose being similar. We first show that, for given $0<\varepsilon<1$,

$$
\lim _{n \rightarrow \infty} P\left(\left\|\sum_{j=1}^{p}\left(\lambda_{j}-\lambda_{0 j}\right) G_{j}\right\|_{R}<1-\varepsilon\right)=1 .
$$

Because $\left\|G_{i}\right\|_{R}=\mathscr{O}_{p}(1)$ (Assumption 21) uniformly in $i,\left(\sum_{j=1}^{p}\left\|G_{j}\right\|_{R}^{2}\right)^{\frac{1}{2}}=\mathscr{O}_{p}(1)$ also, implying that there exists $0<C_{1}<\infty$ such that

$$
\lim _{n \rightarrow \infty} P\left(\left(\sum_{j=1}^{p}\left\|G_{j}\right\|_{R}^{2}\right)^{\frac{1}{2}}>C_{1}\right)=0
$$

The probability on the left side of (B.7) is bounded below by

$$
P\left(\left(\sum_{j=1}^{p}\left(\lambda_{j}-\lambda_{0 j}\right)^{2}\right)^{\frac{1}{2}}\left(\sum_{j=1}^{p}\left\|G_{j}\right\|_{R}^{2}\right)^{\frac{1}{2}}<1-\varepsilon\right)=P\left(\left\|\lambda-\lambda_{0}\right\|\left(\sum_{j=1}^{p}\left\|G_{j}\right\|_{R}^{2}\right)^{\frac{1}{2}}<1-\varepsilon\right),
$$

which in turn is bounded below by

$$
\begin{aligned}
& \quad 1-P\left(\left\|\lambda-\lambda_{0}\right\| \geq C_{1}^{-1}(1-\varepsilon)\right)-P\left(\left(\sum_{j=1}^{p}\left\|G_{j}\right\|_{R}^{2}\right)^{\frac{1}{2}} \geq C_{1}\right) \\
& =P\left(\left\|\lambda-\lambda_{0}\right\|<C_{1}^{-1}(1-\varepsilon)\right)-P\left(\left(\sum_{j=1}^{p}\left\|G_{j}\right\|_{R}^{2}\right)^{\frac{1}{2}} \geq C_{1}\right),
\end{aligned}
$$


where we used the fact that $|c d|>e f$ implies $|c|>e$ or $|d|>f$. Choosing $B\left(\lambda_{0}\right)$ to be a closed subset of a neighbourhood of $\lambda_{0}$ such that $\left\|\lambda-\lambda_{0}\right\|<C_{1}^{-1}(1-\varepsilon)$ implies that the first probability in (B.9) equals 1, while the second probability in (B.9) converges to zero by (B.8). Thus (B.7) is established.

Because $S(\lambda)^{-1}=S^{-1}\left(I_{n}-\sum_{j=1}^{p}\left(\lambda_{j}-\lambda_{0 j}\right) G_{j}\right)^{-1}$, we have

$$
\left\|S(\lambda)^{-1}\right\|_{R} \leq\left\|S^{-1}\right\|_{R}\left\|\left(I_{n}-\sum_{j=1}^{p}\left(\lambda_{j}-\lambda_{0 j}\right) G_{j}\right)^{-1}\right\|_{R},
$$

with the first factor on the RHS of (B.10) $\mathscr{O}_{p}(1)$ by Assumption 21. By (B.7), the second factor equals $\sum_{k=0}^{\infty}\left\|\sum_{j=1}^{p}\left(\lambda_{j}-\lambda_{0 j}\right) G_{j}\right\|_{R}^{k} \leq \sum_{k=0}^{\infty}(1-\varepsilon)^{k}=\varepsilon^{-1}$ with probability one on $B\left(\lambda_{0}\right)$. Thus the lemma is proved.

Lemma B.6. Under the conditions of Theorem 4.2, $G_{i}(\lambda) G_{j}(\lambda)$ and $G_{i}(\lambda) G_{j}(\lambda) G_{k}(\lambda)$ both have elements that are $\mathscr{O}_{p}\left(h_{W}^{-1}\right)$ uniformly in $i, j, k=1, \ldots, p$ and $\lambda \in B\left(\lambda_{0}\right)$.

Proof. We prove the lemma for the triple product, the double product being similarly handled. Take $\lambda \in B\left(\lambda_{0}\right)$. By Assumption 21 and Lemma B.5, $\left\|G_{i}(\lambda)\right\|_{R}$ and $\left\|G_{i}^{\prime}(\lambda)\right\|_{R}$ are uniformly $\mathscr{O}_{p}(1)$ in $B\left(\lambda_{0}\right)$. Let $g_{r, i}^{\prime}(\lambda)$ and $w_{r, i}^{\prime}$ be the $r$-th rows of $G_{i}(\lambda)$ and $W_{i}$ respectively and the $(r, s)$-th element of $G_{i}$ be written as $g_{r s, i}$. Then $g_{r s, i}=w_{r, i}^{\prime} S(\lambda)^{-1} e_{s}=\mathscr{O}_{p}\left(\left\|w_{r, i}\right\|_{R}\right)=\mathscr{O}_{p}\left(h_{W}^{-1}\right)$, uniformly in $r, s=1, \ldots, n$, by Lemma B.5 and Assumption 2. Thus the $(r, s)$-th element of $G_{i}(\lambda) G_{j}(\lambda) G_{k}(\lambda)$, viz. $g_{r, i}^{\prime}(\lambda) G_{j}(\lambda) G_{k}(\lambda) e_{s}$, is $\mathscr{O}_{p}\left(\left\|g_{r, i}\right\|_{R}\right)=\mathscr{O}_{p}\left(\max _{s=1, \ldots, n}\left|g_{r s, i}\right|\right)=\mathscr{O}_{p}\left(h_{W}^{-1}\right)$, uniformly in $r=1, \ldots, n$ and $i, j, k=1, \ldots, p$, as claimed.

\section{References}

Baltagi, B. H., B. Fingleton, and A. Pirotte (2014). Spatial lag models with nested random effects: An instrumental variable procedure with an application to English house prices. Journal of Urban Economics 80, 76-86.

Bell, K. P. and N. E. Bockstael (2000). Applying the generalized-moments estimation approach to spatial problems involving micro-level data. Review of Economics and Statistics 82, 72-82.

Boucher, V. and B. Fortin (2016). Some challenges in the empirics of the effects of networks. In Y. Bramoullé, A. Galeotti, and B. Rogers (Eds.), The Oxford Handbook of the Economics of Networks, Chapter 12. Oxford University Press.

Case, A. C. (1991). Spatial patterns in household demand. Econometrica 59, 953-965.

Cliff, A. D. and J. K. Ord (1973). Spatial Autocorrelation. London: Pion.

Conley, T. G. and B. Dupor (2003). A spatial analysis of sectoral complementarity. Journal of Political Economy 111, 311-352.

Conley, T. G. and E. Ligon (2002). Economic distance and cross-country spillovers. Journal of Economic Growth 7, 157-187.

Das, D., H. H. Kelejian, and I. R. Prucha (2003). Finite sample properties of estimators of spatial autoregressive models with autoregressive disturbances. Papers in Regional Science 82, 1-26.

Davis, P. J. (1979). Circulant Matrices. Wiley Interscience. 
Delgado, M. and P. M. Robinson (2015). Non-nested testing of spatial correlation. Journal of Econometrics 18\%, 385-401.

Gupta, A. and P. M. Robinson (2015). Inference on higher-order spatial autoregressive models with increasingly many parameters. Journal of Econometrics 186, 19-31.

Gupta, A. and P. M. Robinson (2018). Pseudo maximum likelihood estimation of spatial autoregressive models with increasing dimension. Journal of Econometrics 202, 92-107.

Hillier, G. and F. Martellosio (2013). Properties of the maximum likelihood estimator in spatial autoregressive models. Mimeo.

Hillier, G. and F. Martellosio (2018). Exact likelihood inference in group interaction network models. Econometric Theory 34, 383-415.

Jenish, N. and I. R. Prucha (2012). On spatial processes and asymptotic inference under near-epoch dependence. Journal of Econometrics 170, 178 - 190.

Kelejian, H. H. and G. Piras (2014). Estimation of spatial models with endogenous weighting matrices, and an application to a demand model for cigarettes. Regional Science and Urban Economics 46 , 140-149.

Kelejian, H. H. and I. R. Prucha (1998). A generalized spatial two-stage least squares procedure for estimating a spatial autoregressive model with autoregressive disturbances. The Journal of Real Estate Finance and Economics 17, 99-121.

Kelejian, H. H. and I. R. Prucha (1999). A generalized moments estimator for the autoregressive parameter in a spatial model. International Economic Review 40, 509-533.

Kelejian, H. H. and I. R. Prucha (2001). On the asymptotic distribution of the Moran $I$ test statistic with applications. Journal of Econometrics 104, 219-257.

Kelejian, H. H. and I. R. Prucha (2007). HAC estimation in a spatial framework. Journal of Econometrics 140, 131-154.

Kelejian, H. H. and I. R. Prucha (2010). Specification and estimation of spatial autoregressive models with autoregressive and heteroskedastic disturbances. Journal of Econometrics 157, 53-67.

Kuersteiner, G. M. and I. R. Prucha (2013). Limit theory for panel data models with cross sectional dependence and sequential exogeneity. Journal of Econometrics 174, 107-126.

Kuersteiner, G. M. and I. R. Prucha (2015). Dynamic spatial panel models: networks, common shocks, and sequential exogeneity. CESifo Working Paper 5445.

Lee, L. F. (2002). Consistency and efficiency of least squares estimation for mixed regressive, spatial autoregressive models. Econometric Theory 18, 252-277.

Lee, L. F. (2003). Best spatial two-stage least squares estimators for a spatial autoregressive model with autoregressive disturbances. Econometric Reviews 22, 307-335.

Lee, L. F. (2004). Asymptotic distributions of quasi-maximum likelihood estimators for spatial autoregressive models. Econometrica 72, 1899-1925.

Lee, L. F. and X. Liu (2010). Efficient GMM estimation of high order spatial autoregressive models with autoregressive disturbances. Econometric Theory 26, 187-230.

Lee, L. F. and J. Yu (2013). Near unit root in the spatial autoregressive model. Spatial Economic Analysis 8, 314-351.

Lee, L. F. and J. Yu (2014). Efficient GMM estimation of spatial dynamic panel data models with fixed effects. Journal of Econometrics 180, 174-197. 
$\mathrm{Qu}, \mathrm{X}$. and L. F. Lee (2015). Estimating a spatial autoregressive model with an endogenous spatial weight matrix. Journal of Econometrics 184, 209-232.

Robinson, P. M. (2008). Correlation testing in time series, spatial and cross-sectional data. Journal of Econometrics 147, 5-16.

Robinson, P. M. (2010). Efficient estimation of the semiparametric spatial autoregressive model. Journal of Econometrics 157, 6-17.

Robinson, P. M. and F. J. Hidalgo (1997). Time series regression with long-range dependence. The Annals of Statistics 25, 77-104.

Robinson, P. M. and S. Thawornkaiwong (2012). Statistical inference on regression with spatial dependence. Journal of Econometrics 167, 521-542.

Scott, D. J. (1973). Central limit theorems for martingales and for processes with stationary increments using a Skorokhod representation approach. Advances in Applied Probability 5, 119-137.

Souza, P. C. L. (2015). Estimating network effects without network data. Mimeo.

Su, L. and S. Jin (2010). Profile quasi-maximum likelihood estimation of partially linear spatial autoregressive models. Journal of Econometrics 157, 18-33.

$\mathrm{Xu}, \mathrm{X}$. and L. F. Lee (2015). A spatial autoregressive model with a nonlinear transformation of the dependent variable. Journal of Econometrics 186, 1-18.

Yuzefovich, Y. A. (2003). Two Essays on Spatial Econometrics. Ph. D. thesis, University of Maryland. 


\begin{tabular}{|c|c|c|c|c|c|c|c|}
\hline \multirow{2}{*}{ Bias } & \multirow{2}{*}{$\frac{m}{v}$} & \multicolumn{2}{|c|}{48} & \multicolumn{2}{|c|}{72} & \multicolumn{2}{|c|}{144} \\
\hline & & $\lambda$ & $\beta$ & $\lambda$ & $\beta$ & $\lambda$ & $\beta$ \\
\hline \multirow[t]{4}{*}{$\hat{\theta}$} & 1 & 0.0369 & 0.0027 & 0.3397 & 0.0293 & 0.0055 & 0.0048 \\
\hline & 10 & 0.0118 & 0.0285 & 0.0037 & 0.0107 & 0.0052 & 0.0103 \\
\hline & 20 & 0.0175 & 0.0361 & 0.0029 & 0.0117 & 0.0037 & 0.0060 \\
\hline & 100 & 0.0119 & 0.0262 & 0.0083 & 0.0170 & 0.0019 & 0.0030 \\
\hline \multirow[t]{4}{*}{$\tilde{\theta}$} & 1 & 0.0446 & 0.0035 & 0.0313 & 0.0053 & 0.0258 & 0.0064 \\
\hline & 10 & 0.0055 & 0.0044 & 0.0084 & 0.0065 & 0.0009 & 0.0036 \\
\hline & 20 & 0.0048 & 0.0080 & 0.0077 & 0.0037 & 0.0014 & 0.0013 \\
\hline & 100 & 0.0063 & 0.0033 & 0.0032 & 0.0137 & 0.0045 & 0.0063 \\
\hline \multirow[t]{4}{*}{$\check{\theta}$} & 1 & 0.0710 & 0.0051 & 0.0642 & 0.0070 & 0.0601 & 0.0076 \\
\hline & 10 & 0.0443 & 0.0504 & 0.0362 & 0.0462 & 0.0163 & 0.0209 \\
\hline & 20 & 0.0444 & 0.0511 & 0.0357 & 0.0437 & 0.0168 & 0.0236 \\
\hline & 100 & 0.0454 & 0.0557 & 0.0316 & 0.0403 & 0.0198 & 0.0286 \\
\hline MSE & $v$ & $\lambda$ & $\beta$ & $\lambda$ & $\beta$ & $\lambda$ & $\beta$ \\
\hline \multirow[t]{4}{*}{$\hat{\theta}$} & 1 & 0.9217 & 0.0808 & 123.9720 & 0.7877 & 0.7418 & 0.0262 \\
\hline & 10 & 0.0254 & 0.1212 & 0.0164 & 0.0830 & 0.0087 & 0.0410 \\
\hline & 20 & 0.0264 & 0.1272 & 0.0169 & 0.0828 & 0.0081 & 0.0422 \\
\hline & 100 & 0.0259 & 0.1267 & 0.0158 & 0.0818 & 0.0085 & 0.0406 \\
\hline \multirow[t]{4}{*}{$\tilde{\theta}$} & 1 & 0.2254 & 0.0769 & 0.2195 & 0.0532 & 0.2509 & 0.0258 \\
\hline & 10 & 0.0240 & 0.1157 & 0.0163 & 0.0820 & 0.0086 & 0.0405 \\
\hline & 20 & 0.0250 & 0.1225 & 0.0162 & 0.0807 & 0.0079 & 0.0414 \\
\hline & 100 & 0.0248 & 0.1229 & 0.0152 & 0.0801 & 0.0084 & 0.0403 \\
\hline \multirow[t]{4}{*}{$\check{\theta}$} & 1 & 0.1598 & 0.0765 & 0.1638 & 0.0530 & 0.1731 & 0.0257 \\
\hline & 10 & 0.0210 & 0.1071 & 0.0149 & 0.0779 & 0.0080 & 0.0389 \\
\hline & 20 & 0.0216 & 0.1128 & 0.0148 & 0.0765 & 0.0074 & 0.0401 \\
\hline & 100 & 0.0217 & 0.1144 & 0.0137 & 0.0757 & 0.0080 & 0.0392 \\
\hline Size & $v$ & $\lambda$ & $\beta$ & $\lambda$ & $\beta$ & $\lambda$ & $\beta$ \\
\hline \multirow[t]{4}{*}{$\hat{\hat{\theta}}$} & 1 & 0.0490 & 0.0550 & 0.0490 & 0.0625 & 0.0380 & 0.0625 \\
\hline & 10 & 0.0560 & 0.0465 & 0.0520 & 0.0535 & 0.0570 & 0.0475 \\
\hline & 20 & 0.0560 & 0.0640 & 0.0610 & 0.0610 & 0.0460 & 0.0565 \\
\hline & 100 & 0.0560 & 0.0595 & 0.0440 & 0.0460 & 0.0540 & 0.0485 \\
\hline \multirow[t]{4}{*}{$\tilde{\theta}$} & 1 & 0.0610 & 0.0530 & 0.0520 & 0.0645 & 0.0610 & 0.0620 \\
\hline & 10 & 0.0610 & 0.0485 & 0.0560 & 0.0560 & 0.0590 & 0.0475 \\
\hline & 20 & 0.0620 & 0.0640 & 0.0580 & 0.0610 & 0.0470 & 0.0570 \\
\hline & 100 & 0.0580 & 0.0585 & 0.0470 & 0.0495 & 0.0600 & 0.0495 \\
\hline \multirow[t]{4}{*}{$\check{\theta}$} & 1 & 0.0420 & 0.0580 & 0.0310 & 0.0685 & 0.0340 & 0.0640 \\
\hline & 10 & 0.0340 & 0.0445 & 0.0430 & 0.0510 & 0.0530 & 0.0460 \\
\hline & 20 & 0.0420 & 0.0560 & 0.0480 & 0.0550 & 0.0410 & 0.0580 \\
\hline & 100 & 0.0420 & 0.0525 & 0.0360 & 0.0495 & 0.0440 & 0.0470 \\
\hline
\end{tabular}

Table 6.1: Monte Carlo absolute bias, mean squared error and size, nominal size 5\%, dense stochastic $W_{1}, W_{2}$, see section 6.1. $n=2 m$. 


\begin{tabular}{|c|c|c|c|c|c|c|c|}
\hline \multirow{2}{*}{ Bias } & \multirow{2}{*}{$\frac{m}{v}$} & \multicolumn{2}{|c|}{48} & \multicolumn{2}{|c|}{72} & \multicolumn{2}{|c|}{144} \\
\hline & & $\lambda$ & $\beta$ & $\lambda$ & $\beta$ & $\lambda$ & $\beta$ \\
\hline \multirow[t]{4}{*}{$\hat{\theta}$} & 1 & 0.0832 & 0.0103 & 0.0315 & 0.0061 & 0.0296 & 0.0065 \\
\hline & 10 & 0.0058 & 0.0135 & 0.0066 & 0.0126 & 0.0096 & 0.0166 \\
\hline & 20 & 0.0126 & 0.0262 & 0.0040 & 0.0170 & 0.0004 & 0.0063 \\
\hline & 100 & 0.0067 & 0.0153 & 0.0021 & 0.0090 & 0.0059 & 0.0137 \\
\hline \multirow[t]{4}{*}{$\tilde{\theta}$} & 1 & 0.0473 & 0.0100 & 0.0286 & 0.0052 & 0.0210 & 0.0067 \\
\hline & 10 & 0.0127 & 0.0127 & 0.0051 & 0.0041 & 0.0034 & 0.0077 \\
\hline & 20 & 0.0040 & 0.0030 & 0.0063 & 0.0021 & 0.0048 & 0.0016 \\
\hline & 100 & 0.0112 & 0.0104 & 0.0131 & 0.0107 & 0.0006 & 0.0044 \\
\hline \multirow[t]{4}{*}{$\check{\theta}$} & 1 & 0.1069 & 0.0099 & 0.0750 & 0.0053 & 0.0807 & 0.0067 \\
\hline & 10 & 0.0498 & 0.0656 & 0.0327 & 0.0437 & 0.0123 & 0.0150 \\
\hline & 20 & 0.0431 & 0.0524 & 0.0344 & 0.0379 & 0.0203 & 0.0237 \\
\hline & 100 & 0.0492 & 0.0644 & 0.0408 & 0.0505 & 0.0162 & 0.0181 \\
\hline MSE & $v$ & $\lambda$ & $\beta$ & $\lambda$ & $\beta$ & $\lambda$ & $\beta$ \\
\hline \multirow[t]{4}{*}{$\hat{\theta}$} & 1 & 1.9703 & 0.0770 & 3.6357 & 0.0482 & 1.3637 & 0.0240 \\
\hline & 10 & 0.0247 & 0.1198 & 0.0150 & 0.0795 & 0.0081 & 0.0427 \\
\hline & 20 & 0.0263 & 0.1264 & 0.0168 & 0.0820 & 0.0091 & 0.0401 \\
\hline & 100 & 0.0243 & 0.1213 & 0.0184 & 0.0839 & 0.0083 & 0.0412 \\
\hline \multirow[t]{4}{*}{$\tilde{\theta}$} & 1 & 0.3590 & 0.0756 & 0.3343 & 0.0447 & 0.4064 & 0.0232 \\
\hline & 10 & 0.0243 & 0.1172 & 0.0149 & 0.0787 & 0.0078 & 0.0417 \\
\hline & 20 & 0.0255 & 0.1225 & 0.0164 & 0.0808 & 0.0090 & 0.0399 \\
\hline & 100 & 0.0231 & 0.1181 & 0.0187 & 0.0838 & 0.0083 & 0.0409 \\
\hline \multirow[t]{4}{*}{$\check{\theta}$} & 1 & 0.2100 & 0.0749 & 0.2198 & 0.0446 & 0.2497 & 0.0231 \\
\hline & 10 & 0.0219 & 0.1107 & 0.0136 & 0.0750 & 0.0072 & 0.0400 \\
\hline & 20 & 0.0220 & 0.1130 & 0.0150 & 0.0763 & 0.0086 & 0.0384 \\
\hline & 100 & 0.0207 & 0.1115 & 0.0173 & 0.0797 & 0.0078 & 0.0393 \\
\hline Size & $v$ & $\lambda$ & $\beta$ & $\lambda$ & $\beta$ & $\lambda$ & $\beta$ \\
\hline \multirow[t]{4}{*}{$\hat{\theta}$} & 1 & 0.0590 & 0.0515 & 0.0550 & 0.0425 & 0.0500 & 0.0480 \\
\hline & 10 & 0.0470 & 0.0540 & 0.0400 & 0.0500 & 0.0480 & 0.0535 \\
\hline & 20 & 0.0600 & 0.0560 & 0.0550 & 0.0570 & 0.0540 & 0.0455 \\
\hline & 100 & 0.0540 & 0.0490 & 0.0590 & 0.0520 & 0.0480 & 0.0500 \\
\hline \multirow[t]{4}{*}{$\tilde{\theta}$} & 1 & 0.0750 & 0.0515 & 0.0540 & 0.0450 & 0.0540 & 0.0510 \\
\hline & 10 & 0.0610 & 0.0550 & 0.0460 & 0.0515 & 0.0490 & 0.0535 \\
\hline & 20 & 0.0640 & 0.0595 & 0.0530 & 0.0555 & 0.0540 & 0.0445 \\
\hline & 100 & 0.0540 & 0.0500 & 0.0700 & 0.0525 & 0.0460 & 0.0495 \\
\hline \multirow[t]{4}{*}{$\check{\theta}$} & 1 & 0.0290 & 0.0560 & 0.0270 & 0.0460 & 0.0200 & 0.0505 \\
\hline & 10 & 0.0510 & 0.0505 & 0.0320 & 0.0485 & 0.0380 & 0.0510 \\
\hline & 20 & 0.0480 & 0.0565 & 0.0420 & 0.0490 & 0.0470 & 0.0435 \\
\hline & 100 & 0.0440 & 0.0475 & 0.0600 & 0.0510 & 0.0430 & 0.0465 \\
\hline
\end{tabular}

Table 6.2: Monte Carlo absolute bias, mean squared error and size, nominal size 5\%, dense fixed $W_{1}, W_{2}$, see section 6.1. $n=2 m$. 


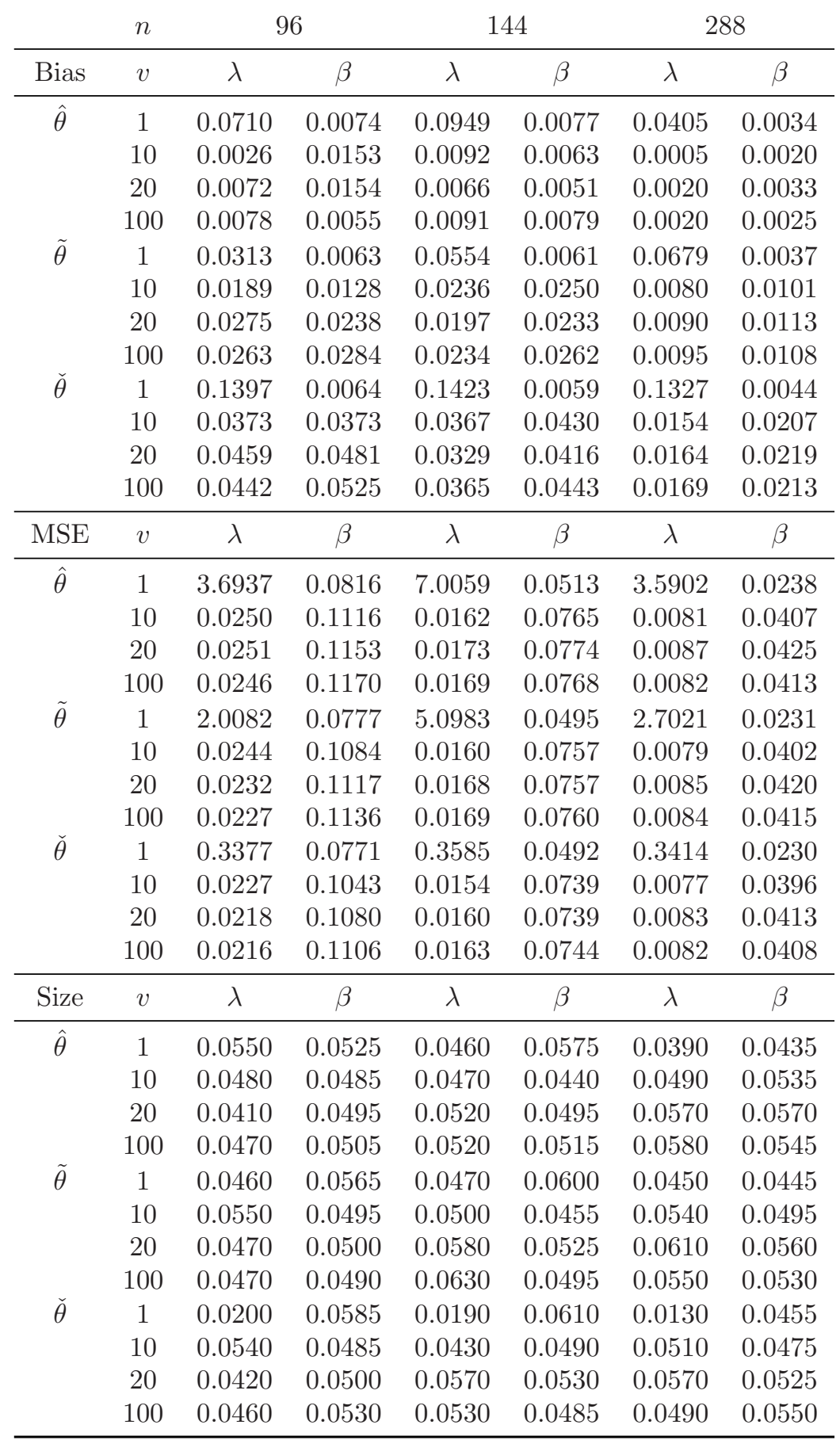

Table 6.3: Monte Carlo absolute bias, mean squared error and size, nominal size 5\%, sparse stochastic $W$, see Section 6.1. 


\begin{tabular}{|c|c|c|c|c|c|c|c|}
\hline \multirow{2}{*}{ Bias } & \multirow{2}{*}{$\begin{array}{l}n \\
v\end{array}$} & \multicolumn{2}{|c|}{96} & \multicolumn{2}{|c|}{144} & \multicolumn{2}{|c|}{288} \\
\hline & & $\lambda$ & $\beta$ & $\lambda$ & $\beta$ & $\lambda$ & $\beta$ \\
\hline \multirow[t]{4}{*}{$\hat{\theta}$} & 1 & 0.2813 & 0.0077 & 0.0213 & 0.0019 & 0.0354 & 0.0033 \\
\hline & 10 & 0.0000 & 0.0082 & 0.0033 & 0.0031 & 0.0073 & 0.0066 \\
\hline & 20 & 0.0011 & 0.0063 & 0.0045 & 0.0042 & 0.0025 & 0.0017 \\
\hline & 100 & 0.0066 & 0.0041 & 0.0016 & 0.0083 & 0.0040 & 0.0054 \\
\hline \multirow[t]{4}{*}{$\tilde{\theta}$} & 1 & 0.0021 & 0.0109 & 0.0106 & 0.0025 & 0.0115 & 0.0031 \\
\hline & 10 & 0.0231 & 0.0224 & 0.0186 & 0.0208 & 0.0149 & 0.0173 \\
\hline & 20 & 0.0180 & 0.0170 & 0.0183 & 0.0149 & 0.0101 & 0.0124 \\
\hline & 100 & 0.0271 & 0.0258 & 0.0174 & 0.0155 & 0.0116 & 0.0095 \\
\hline \multirow[t]{4}{*}{$\check{\theta}$} & 1 & 0.1725 & 0.0114 & 0.0391 & 0.0040 & 0.0518 & 0.0031 \\
\hline & 10 & 0.0415 & 0.0469 & 0.0316 & 0.0386 & 0.0222 & 0.0276 \\
\hline & 20 & 0.0371 & 0.0428 & 0.0320 & 0.0337 & 0.0174 & 0.0227 \\
\hline & 100 & 0.0462 & 0.0517 & 0.0311 & 0.0346 & 0.0191 & 0.0202 \\
\hline MSE & $v$ & $\lambda$ & $\beta$ & $\lambda$ & $\beta$ & $\lambda$ & $\beta$ \\
\hline \multirow[t]{4}{*}{$\hat{\theta}$} & 1 & 33.4155 & 0.0878 & 0.6563 & 0.0528 & 1.9369 & 0.0252 \\
\hline & 10 & 0.0254 & 0.1131 & 0.0153 & 0.0757 & 0.0084 & 0.0392 \\
\hline & 20 & 0.0252 & 0.1135 & 0.0177 & 0.0810 & 0.0081 & 0.0390 \\
\hline & 100 & 0.0250 & 0.1179 & 0.0168 & 0.0787 & 0.0093 & 0.0438 \\
\hline \multirow[t]{4}{*}{$\tilde{\theta}$} & 1 & 2.3193 & 0.0744 & 0.1910 & 0.0510 & 0.2607 & 0.0240 \\
\hline & 10 & 0.0245 & 0.1099 & 0.0150 & 0.0741 & 0.0084 & 0.0390 \\
\hline & 20 & 0.0238 & 0.1102 & 0.0173 & 0.0796 & 0.0081 & 0.0389 \\
\hline & 100 & 0.0244 & 0.1153 & 0.0159 & 0.0761 & 0.0090 & 0.0428 \\
\hline \multirow[t]{4}{*}{$\check{\theta}$} & 1 & 0.4626 & 0.0733 & 0.1493 & 0.0508 & 0.1846 & 0.0239 \\
\hline & 10 & 0.0229 & 0.1059 & 0.0144 & 0.0724 & 0.0083 & 0.0386 \\
\hline & 20 & 0.0219 & 0.1058 & 0.0165 & 0.0771 & 0.0079 & 0.0383 \\
\hline & 100 & 0.0228 & 0.1113 & 0.0152 & 0.0739 & 0.0088 & 0.0421 \\
\hline Size & $v$ & $\lambda$ & $\beta$ & $\lambda$ & $\beta$ & $\lambda$ & $\beta$ \\
\hline \multirow[t]{4}{*}{$\hat{\theta}$} & 1 & 0.0450 & 0.0480 & 0.0510 & 0.0570 & 0.0340 & 0.0485 \\
\hline & 10 & 0.0550 & 0.0460 & 0.0480 & 0.0505 & 0.0420 & 0.0475 \\
\hline & 20 & 0.0510 & 0.0485 & 0.0550 & 0.0595 & 0.0450 & 0.0535 \\
\hline & 100 & 0.0500 & 0.0490 & 0.0540 & 0.0525 & 0.0580 & 0.0575 \\
\hline \multirow[t]{4}{*}{$\tilde{\theta}$} & 1 & 0.0470 & 0.0490 & 0.0500 & 0.0585 & 0.0560 & 0.0510 \\
\hline & 10 & 0.0650 & 0.0535 & 0.0580 & 0.0490 & 0.0470 & 0.0530 \\
\hline & 20 & 0.0490 & 0.0465 & 0.0580 & 0.0600 & 0.0470 & 0.0545 \\
\hline & 100 & 0.0570 & 0.0495 & 0.0590 & 0.0520 & 0.0530 & 0.0560 \\
\hline \multirow[t]{4}{*}{$\check{\theta}$} & 1 & 0.0140 & 0.0500 & 0.0270 & 0.0620 & 0.0250 & 0.0520 \\
\hline & 10 & 0.0550 & 0.0465 & 0.0530 & 0.0500 & 0.0480 & 0.0505 \\
\hline & 20 & 0.0410 & 0.0475 & 0.0550 & 0.0575 & 0.0490 & 0.0530 \\
\hline & 100 & 0.0550 & 0.0495 & 0.0540 & 0.0490 & 0.0500 & 0.0590 \\
\hline
\end{tabular}

Table 6.4: Monte Carlo absolute bias, mean squared error and size, nominal size 5\%, sparse fixed $W$, see Section 6.1 . 


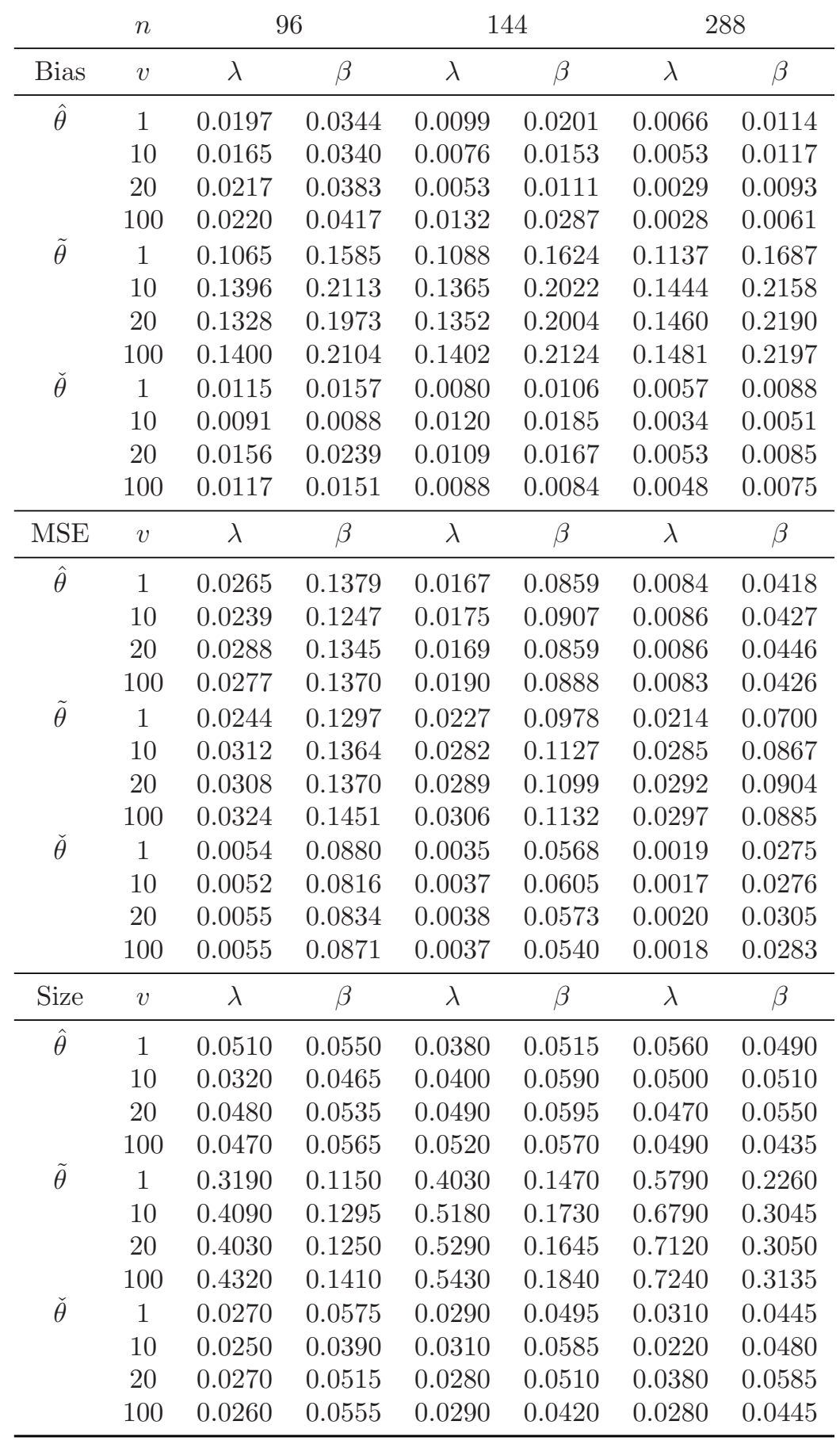

Table 6.5: Monte Carlo absolute bias, mean squared error and size, nominal size 5\%, circulant stochastic $W$, see Section 6.1. 


\begin{tabular}{|c|c|c|c|c|c|c|c|}
\hline \multirow{2}{*}{ Bias } & \multirow{2}{*}{$\begin{array}{l}n \\
v\end{array}$} & \multicolumn{2}{|c|}{96} & \multicolumn{2}{|c|}{144} & \multicolumn{2}{|c|}{288} \\
\hline & & $\lambda$ & $\beta$ & $\lambda$ & $\beta$ & $\lambda$ & $\beta$ \\
\hline \multirow[t]{4}{*}{$\hat{\theta}$} & 1 & 0.0163 & 0.0341 & 0.0068 & 0.0163 & 0.0043 & 0.0074 \\
\hline & 10 & 0.0093 & 0.0171 & 0.0055 & 0.0129 & 0.0041 & 0.0073 \\
\hline & 20 & 0.0181 & 0.0338 & 0.0122 & 0.0305 & 0.0061 & 0.0150 \\
\hline & 100 & 0.0121 & 0.0268 & 0.0066 & 0.0198 & 0.0075 & 0.0153 \\
\hline \multirow[t]{4}{*}{$\tilde{\theta}$} & 1 & 0.1319 & 0.1984 & 0.1980 & 0.2958 & 0.1404 & 0.2072 \\
\hline & 10 & 0.0905 & 0.1320 & 0.1210 & 0.1798 & 0.0014 & 0.0066 \\
\hline & 20 & 0.1185 & 0.1771 & 0.2092 & 0.3162 & 0.0857 & 0.1299 \\
\hline & 100 & 0.0240 & 0.0399 & 0.1342 & 0.2028 & 0.2191 & 0.3284 \\
\hline \multirow[t]{4}{*}{$\check{\theta}$} & 1 & 0.0122 & 0.0144 & 0.0098 & 0.0130 & 0.0057 & 0.0096 \\
\hline & 10 & 0.0139 & 0.0228 & 0.0099 & 0.0137 & 0.0054 & 0.0078 \\
\hline & 20 & 0.0123 & 0.0164 & 0.0101 & 0.0085 & 0.0037 & 0.0053 \\
\hline & 100 & 0.0152 & 0.0174 & 0.0091 & 0.0078 & 0.0018 & 0.0016 \\
\hline MSE & $v$ & $\lambda$ & $\beta$ & $\lambda$ & $\beta$ & $\lambda$ & $\beta$ \\
\hline \multirow[t]{4}{*}{$\hat{\theta}$} & 1 & 0.0282 & 0.1380 & 0.0174 & 0.0851 & 0.0090 & 0.0450 \\
\hline & 10 & 0.0249 & 0.1247 & 0.0168 & 0.0841 & 0.0078 & 0.0436 \\
\hline & 20 & 0.0267 & 0.1352 & 0.0192 & 0.0904 & 0.0082 & 0.0426 \\
\hline & 100 & 0.0234 & 0.1178 & 0.0175 & 0.0840 & 0.0091 & 0.0418 \\
\hline \multirow[t]{4}{*}{$\tilde{\theta}$} & 1 & 0.0249 & 0.1270 & 0.0454 & 0.1451 & 0.0224 & 0.0727 \\
\hline & 10 & 0.0149 & 0.0987 & 0.0193 & 0.0887 & 0.0019 & 0.0307 \\
\hline & 20 & 0.0217 & 0.1220 & 0.0496 & 0.1586 & 0.0097 & 0.0464 \\
\hline & 100 & 0.0069 & 0.0805 & 0.0233 & 0.0986 & 0.0508 & 0.1348 \\
\hline \multirow[t]{4}{*}{$\check{\theta}$} & 1 & 0.0055 & 0.0848 & 0.0039 & 0.0546 & 0.0019 & 0.0290 \\
\hline & 10 & 0.0054 & 0.0803 & 0.0035 & 0.0550 & 0.0019 & 0.0307 \\
\hline & 20 & 0.0057 & 0.0877 & 0.0036 & 0.0569 & 0.0019 & 0.0292 \\
\hline & 100 & 0.0060 & 0.0779 & 0.0039 & 0.0554 & 0.0017 & 0.0257 \\
\hline Size & $v$ & $\lambda$ & $\beta$ & $\lambda$ & $\beta$ & $\lambda$ & $\beta$ \\
\hline \multirow[t]{4}{*}{$\hat{\theta}$} & 1 & 0.0560 & 0.0510 & 0.0410 & 0.0455 & 0.0580 & 0.0580 \\
\hline & 10 & 0.0400 & 0.0420 & 0.0440 & 0.0475 & 0.0450 & 0.0600 \\
\hline & 20 & 0.0410 & 0.0535 & 0.0420 & 0.0555 & 0.0440 & 0.0490 \\
\hline & 100 & 0.0400 & 0.0400 & 0.0470 & 0.0530 & 0.0460 & 0.0405 \\
\hline \multirow[t]{4}{*}{$\tilde{\theta}$} & 1 & 0.3960 & 0.1195 & 0.7600 & 0.2465 & 0.8050 & 0.2465 \\
\hline & 10 & 0.2300 & 0.0735 & 0.4330 & 0.1230 & 0.0550 & 0.0625 \\
\hline & 20 & 0.3430 & 0.0990 & 0.7960 & 0.2810 & 0.4840 & 0.1240 \\
\hline & 100 & 0.0790 & 0.0415 & 0.5220 & 0.1410 & 0.9780 & 0.5080 \\
\hline \multirow[t]{4}{*}{$\check{\theta}$} & 1 & 0.0230 & 0.0475 & 0.0210 & 0.0430 & 0.0360 & 0.0550 \\
\hline & 10 & 0.0270 & 0.0435 & 0.0240 & 0.0405 & 0.0540 & 0.0615 \\
\hline & 20 & 0.0270 & 0.0530 & 0.0150 & 0.0425 & 0.0410 & 0.0595 \\
\hline & 100 & 0.0510 & 0.0440 & 0.0320 & 0.0500 & 0.0170 & 0.0340 \\
\hline
\end{tabular}

Table 6.6: Monte Carlo absolute bias, mean squared error and size, nominal size 5\%, circulant fixed $W$, see Section 6.1. 


\begin{tabular}{ccccccc}
$n$ & \multicolumn{2}{c}{48} & \multicolumn{2}{c}{96} & \multicolumn{2}{c}{144} \\
\hline Bias & $\lambda$ & $\beta$ & $\lambda$ & $\beta$ & $\lambda$ & $\beta$ \\
\hline$\hat{\theta}$ & 0.0244 & 0.0058 & 0.0025 & 0.0060 & 0.0098 & 0.0042 \\
$\tilde{\theta}$ & 0.1792 & 0.0804 & 0.0754 & 0.0384 & 0.0399 & 0.0255 \\
$\check{\theta}$ & 0.1747 & 0.1131 & 0.0832 & 0.0576 & 0.0463 & 0.0388 \\
\hline $\mathrm{MSE}$ & $\lambda$ & $\beta$ & $\lambda$ & $\beta$ & $\lambda$ & $\beta$ \\
\hline$\hat{\theta}$ & 0.1898 & 0.1487 & 0.0812 & 0.0696 & 0.0556 & 0.0464 \\
$\tilde{\theta}$ & 0.2385 & 0.1706 & 0.0905 & 0.0741 & 0.0587 & 0.0486 \\
$\check{\theta}$ & 0.1852 & 0.1598 & 0.0852 & 0.0727 & 0.0565 & 0.0479 \\
\hline Size & $\lambda$ & $\beta$ & $\lambda$ & $\beta$ & $\lambda$ & $\beta$ \\
\hline$\hat{\theta}$ & 0.0570 & 0.0590 & 0.0525 & 0.0480 & 0.0510 & 0.0500 \\
$\tilde{\theta}$ & 0.0860 & 0.0710 & 0.0530 & 0.0560 & 0.0555 & 0.0505 \\
$\check{\theta}$ & 0.0585 & 0.0670 & 0.0485 & 0.0545 & 0.0515 & 0.0550 \\
\hline
\end{tabular}

Table 6.7: Monte Carlo absolute (average) bias, mean squared error and size, nominal size $5 \%, W_{1}$ and $W_{2}$ generated as in Section 6.2 using (6.2) and (6.3) respectively. 


\title{
Supplementary Material to 'Estimation of spatial autoregressions with stochastic weight matrices'*
}

\author{
Abhimanyu Gupta ${ }^{\dagger}$ \\ Department of Economics \\ University of Essex
}

March 14, 2018

\section{A simulation study when spatial weights are generated by de- pendent random variables}

Section 6.1 of 'Estimation of spatial autoregressions with stochastic weight matrices' took the spatial weights to be iid but in some cases, especially for asymmetric spatial weight matrices, this may not be reasonable. For instance, if the distance from unit $r$ to $s$ is small the distance from $s$ to $r$ may also be expected to be small. To capture such behaviour we use the same designs as described in Section 6.1, but with the following alteration: after generating the $V_{j}$, replace $v_{r s, j}=\left(v_{s r, j}^{2}+5\right)^{\frac{1}{2}}$ for each $r=1, \ldots, m$ and $s \leq r$, where $v_{r s, j}$ denotes the $(r, s)$-th element of $V_{j}$. Thus we replace the part of $V_{j}$ below the diagonal with a transformation of the part above the diagonal. The choice of transformation is uniformly continuous, in keeping with the idea of 'preserving' the distance between units discussed earlier in the paragraph. Similar operations are carried out with the sparse and circulant specifications of $W$. We then proceed with the experiment design as in the corresponding parts of Section 6.1.

The results are in Tables 0.1(a)-(c), where we report the stochastic case. They indicate that the procedure of generating dependent weights in this way does little to alter the character and behaviour of the estimates. The same features that we saw in Tables 6.1, 6.3 and 6.5 are evident. We may also compare the dense, sparse and circulant cases to see if stochastic dependent spatial weights yield any difference in performance as opposed to stochastic iid ones. Out of 72 comparisons for each type of weight matrix, the dependent setting exhibits a smaller bias in 54 (dense), 61 (sparse) and 41 (circulant) cases, while the MSE is smaller in all 72 (dense), 54 (sparse) and 32 (circulant) cases. Thus in our experiment designs dependent spatial weights do not contaminate the performance of estimates.

*This appendix should be read in conjunction with Section 6 of 'Estimation of spatial autoregressions with stochastic weight matrices'.

†Email: a.gupta@essex.ac.uk. 
(a) Dense $W_{1}, W_{2}$

(b) Sparse $W$

(c) Circulant $W$

$m / n$ 72 144 96 144 288 96 288

\begin{tabular}{|c|c|c|c|c|c|c|c|c|c|c|c|c|c|c|c|c|c|c|c|}
\hline \multirow[b]{2}{*}{ Bias } & \multirow{2}{*}{$\begin{array}{c}m / n \\
v \\
\end{array}$} & \multicolumn{2}{|c|}{48} & \multicolumn{2}{|c|}{72} & \multicolumn{2}{|c|}{144} & \multicolumn{2}{|c|}{96} & \multicolumn{2}{|c|}{144} & \multicolumn{2}{|c|}{288} & \multicolumn{2}{|c|}{96} & \multicolumn{2}{|c|}{144} & \multicolumn{2}{|c|}{288} \\
\hline & & $\lambda$ & $\beta$ & $\lambda$ & $\beta$ & $\lambda$ & $\beta$ & $\lambda$ & $\beta$ & $\lambda$ & $\beta$ & $\lambda$ & $\beta$ & $\lambda$ & $\beta$ & $\lambda$ & $\beta$ & $\lambda$ & $\beta$ \\
\hline \multirow[t]{4}{*}{$\hat{\theta}$} & 1 & 0.0292 & 0.0025 & 0.0423 & 0.0046 & 0.0459 & 0.0009 & 0.0280 & 0.0061 & 0.0585 & 0.0059 & 0.0586 & 0.0038 & 0.0058 & 0.0309 & 0.0024 & 0.0151 & 0.0027 & 0.0152 \\
\hline & 10 & 0.0126 & 0.0143 & 0.0059 & 0.0069 & 0.0051 & 0.0046 & 0.0017 & 0.0063 & 0.0062 & 0.0062 & 0.0005 & 0.0009 & 0.0047 & 0.0367 & 0.0017 & 0.0139 & 0.0015 & 0.0128 \\
\hline & 20 & 0.0086 & 0.0104 & 0.0067 & 0.0104 & 0.0016 & 0.0050 & 0.0033 & 0.0151 & 0.0027 & 0.0046 & 0.0001 & 0.0031 & 0.0066 & 0.0422 & 0.0024 & 0.0099 & 0.0001 & 0.0074 \\
\hline & 100 & 0.0036 & 0.0030 & 0.0023 & 0.0084 & 0.0012 & 0.0013 & 0.0100 & 0.0109 & 0.0046 & 0.0076 & 0.0021 & 0.0027 & 0.0055 & 0.0369 & 0.0047 & 0.0346 & 0.0003 & 0.0044 \\
\hline \multirow[t]{4}{*}{$\tilde{\theta}$} & 1 & 0.0698 & 0.0151 & 0.0622 & 0.0084 & 0.0793 & 0.0066 & 0.0008 & 0.0063 & 0.0396 & 0.0055 & 0.0417 & 0.0039 & 0.0697 & 0.4591 & 0.0769 & 0.4970 & 0.0874 & 0.5451 \\
\hline & 10 & 0.0007 & 0.0021 & 0.0016 & 0.0020 & 0.0012 & 0.0009 & 0.0091 & 0.0052 & 0.0116 & 0.0107 & 0.0031 & 0.0049 & 0.0810 & 0.5066 & 0.0908 & 0.5429 & 0.1008 & 0.5947 \\
\hline & 20 & 0.0036 & 0.0038 & 0.0017 & 0.0032 & 0.0031 & 0.0005 & 0.0125 & 0.0154 & 0.0090 & 0.0116 & 0.0027 & 0.0044 & 0.0819 & 0.5032 & 0.0889 & 0.5336 & 0.1000 & 0.5902 \\
\hline & 100 & 0.0104 & 0.0131 & 0.0052 & 0.0084 & 0.0058 & 0.0050 & 0.0207 & 0.0226 & 0.0120 & 0.0117 & 0.0016 & 0.0026 & 0.0811 & 0.5006 & 0.0896 & 0.5452 & 0.0994 & 0.5836 \\
\hline \multirow[t]{4}{*}{$\check{\theta}$} & 1 & 0.0516 & 0.0073 & 0.0625 & 0.0078 & 0.0531 & 0.0029 & 0.1230 & 0.0081 & 0.1311 & 0.0076 & 0.1335 & 0.0063 & 0.0008 & 0.0102 & 0.0034 & 0.0204 & 0.0090 & 0.0548 \\
\hline & 10 & 0.0155 & 0.0187 & 0.0116 & 0.0137 & 0.0039 & 0.0062 & 0.0306 & 0.0280 & 0.0263 & 0.0265 & 0.0107 & 0.0132 & 0.0048 & 0.0315 & 0.0059 & 0.0339 & 0.0118 & 0.0715 \\
\hline & 20 & 0.0176 & 0.0202 & 0.0113 & 0.0106 & 0.0080 & 0.0063 & 0.0336 & 0.0292 & 0.0231 & 0.0268 & 0.0100 & 0.0124 & 0.0040 & 0.0222 & 0.0065 & 0.0363 & 0.0122 & 0.0749 \\
\hline & 100 & 0.0240 & 0.0287 & 0.0144 & 0.0177 & 0.0105 & 0.0104 & 0.0402 & 0.0430 & 0.0258 & 0.0264 & 0.0087 & 0.0094 & 0.0037 & 0.0225 & 0.0080 & 0.0505 & 0.0135 & 0.0823 \\
\hline MSE & $v$ & $\lambda$ & $\beta$ & $\lambda$ & $\beta$ & $\lambda$ & $\beta$ & $\lambda$ & $\beta$ & $\lambda$ & $\beta$ & $\lambda$ & $\beta$ & $\lambda$ & $\beta$ & $\lambda$ & $\beta$ & $\lambda$ & $\beta$ \\
\hline \multirow[t]{4}{*}{$\hat{\theta}$} & 1 & 0.1665 & 0.0398 & 0.1529 & 0.0245 & 0.3150 & 0.0131 & 1.9196 & 0.0822 & 0.9467 & 0.0518 & 1.3855 & 0.0242 & 0.0048 & 0.2282 & 0.0028 & 0.1434 & 0.0016 & 0.0746 \\
\hline & 10 & 0.0119 & 0.0530 & 0.0083 & 0.0350 & 0.0039 & 0.0171 & 0.0257 & 0.0998 & 0.0158 & 0.0665 & 0.0077 & 0.0339 & 0.0039 & 0.2033 & 0.0033 & 0.1548 & 0.0018 & 0.0797 \\
\hline & 20 & 0.0120 & 0.0530 & 0.0085 & 0.0358 & 0.0043 & 0.0174 & 0.0269 & 0.1028 & 0.0167 & 0.0650 & 0.0080 & 0.0346 & 0.0043 & 0.2106 & 0.0038 & 0.1583 & 0.0018 & 0.0782 \\
\hline & 100 & 0.0120 & 0.0534 & 0.0076 & 0.0335 & 0.0040 & 0.0177 & 0.0235 & 0.1031 & 0.0171 & 0.0646 & 0.0078 & 0.0335 & 0.0042 & 0.2075 & 0.0033 & 0.1530 & 0.0017 & 0.0783 \\
\hline \multirow[t]{4}{*}{$\tilde{\theta}$} & 1 & 0.0461 & 0.0395 & 0.0408 & 0.0241 & 0.0515 & 0.0130 & 0.5264 & 0.0784 & 0.6161 & 0.0502 & 0.5338 & 0.0233 & 0.0069 & 0.3462 & 0.0081 & 0.3464 & 0.0099 & 0.3710 \\
\hline & 10 & 0.0117 & 0.0525 & 0.0084 & 0.0350 & 0.0039 & 0.0171 & 0.0266 & 0.1002 & 0.0156 & 0.0662 & 0.0077 & 0.0338 & 0.0084 & 0.3664 & 0.0102 & 0.3831 & 0.0119 & 0.4084 \\
\hline & 20 & 0.0118 & 0.0525 & 0.0084 & 0.0356 & 0.0042 & 0.0172 & 0.0261 & 0.1024 & 0.0166 & 0.0649 & 0.0080 & 0.0346 & 0.0086 & 0.3675 & 0.0098 & 0.3736 & 0.0116 & 0.4051 \\
\hline & 100 & 0.0120 & 0.0532 & 0.0076 & 0.0336 & 0.0040 & 0.0177 & 0.0229 & 0.1024 & 0.0169 & 0.0643 & 0.0078 & 0.0335 & 0.0085 & 0.3699 & 0.0098 & 0.3818 & 0.0115 & 0.3975 \\
\hline \multirow[t]{4}{*}{$\check{\theta}$} & 1 & 0.0287 & 0.0387 & 0.0301 & 0.0240 & 0.0302 & 0.0128 & 0.1552 & 0.0769 & 0.1628 & 0.0495 & 0.1677 & 0.0231 & 0.0016 & 0.1481 & 0.0017 & 0.1136 & 0.0020 & 0.1014 \\
\hline & 10 & 0.0109 & 0.0512 & 0.0081 & 0.0344 & 0.0038 & 0.0169 & 0.0243 & 0.0970 & 0.0150 & 0.0651 & 0.0075 & 0.0335 & 0.0023 & 0.1610 & 0.0023 & 0.1392 & 0.0027 & 0.1235 \\
\hline & 20 & 0.0111 & 0.0514 & 0.0081 & 0.0349 & 0.0041 & 0.0170 & 0.0242 & 0.0996 & 0.0158 & 0.0639 & 0.0078 & 0.0343 & 0.0024 & 0.1635 & 0.0024 & 0.1372 & 0.0027 & 0.1279 \\
\hline & 100 & 0.0115 & 0.0524 & 0.0073 & 0.0331 & 0.0040 & 0.0176 & 0.0217 & 0.1008 & 0.0162 & 0.0634 & 0.0076 & 0.0332 & 0.0022 & 0.1606 & 0.0025 & 0.1415 & 0.0029 & 0.1312 \\
\hline Size & $v$ & $\lambda$ & $\beta$ & $\lambda$ & $\beta$ & $\lambda$ & $\beta$ & $\lambda$ & $\beta$ & $\lambda$ & $\beta$ & $\lambda$ & $\beta$ & $\lambda$ & $\beta$ & $\lambda$ & $\beta$ & $\lambda$ & $\beta$ \\
\hline \multirow[t]{4}{*}{$\hat{\theta}$} & 1 & 0.0430 & 0.0575 & 0.0330 & 0.0475 & 0.0480 & 0.0580 & 0.0540 & 0.0555 & 0.0530 & 0.0565 & 0.0500 & 0.0415 & 0.0530 & 0.0540 & 0.0380 & 0.0415 & 0.0560 & 0.0535 \\
\hline & 10 & 0.0610 & 0.0565 & 0.0640 & 0.0470 & 0.0440 & 0.0430 & 0.0490 & 0.0445 & 0.0410 & 0.0480 & 0.0420 & 0.0535 & 0.0520 & 0.0440 & 0.0470 & 0.0580 & 0.0490 & 0.0605 \\
\hline & 20 & 0.0510 & 0.0550 & 0.0500 & 0.0555 & 0.0660 & 0.0500 & 0.0560 & 0.0565 & 0.0500 & 0.0415 & 0.0460 & 0.0535 & 0.0470 & 0.0520 & 0.0460 & 0.0510 & 0.0430 & 0.0445 \\
\hline & 100 & 0.0510 & 0.0570 & 0.0450 & 0.0435 & 0.0490 & 0.0520 & 0.0470 & 0.0530 & 0.0550 & 0.0505 & 0.0490 & 0.0535 & 0.0470 & 0.0540 & 0.0450 & 0.0505 & 0.0450 & 0.0455 \\
\hline \multirow[t]{4}{*}{$\tilde{\theta}$} & 1 & 0.1040 & 0.0585 & 0.0760 & 0.0455 & 0.1070 & 0.0615 & 0.0640 & 0.0535 & 0.0740 & 0.0610 & 0.0710 & 0.0430 & 0.8110 & 0.4335 & 0.8890 & 0.6105 & 0.9390 & 0.8100 \\
\hline & 10 & 0.0560 & 0.0570 & 0.0640 & 0.0480 & 0.0440 & 0.0425 & 0.0580 & 0.0485 & 0.0390 & 0.0485 & 0.0490 & 0.0525 & 0.9070 & 0.4670 & 0.9460 & 0.6540 & 0.9840 & 0.8845 \\
\hline & 20 & 0.0560 & 0.0540 & 0.0550 & 0.0535 & 0.0590 & 0.0510 & 0.0620 & 0.0570 & 0.0500 & 0.0425 & 0.0530 & 0.0555 & 0.8930 & 0.4820 & 0.9380 & 0.6420 & 0.9760 & 0.8800 \\
\hline & 100 & 0.0590 & 0.0570 & 0.0490 & 0.0440 & 0.0480 & 0.0520 & 0.0440 & 0.0520 & 0.0550 & 0.0505 & 0.0520 & 0.0515 & 0.8920 & 0.4785 & 0.9430 & 0.6730 & 0.9790 & 0.8735 \\
\hline$\check{\theta}$ & 1 & 0.0210 & 0.0570 & 0.0150 & 0.0465 & 0.0160 & 0.0585 & 0.0240 & 0.0545 & 0.0130 & 0.0630 & 0.0120 & 0.0435 & 0.1120 & 0.1040 & 0.1260 & 0.1000 & 0.1510 & 0.1210 \\
\hline & 10 & 0.0430 & 0.0560 & 0.0600 & 0.0455 & 0.0390 & 0.0445 & 0.0520 & 0.0510 & 0.0360 & 0.0510 & 0.0420 & 0.0520 & 0.1310 & 0.1095 & 0.1560 & 0.1330 & 0.1730 & 0.1475 \\
\hline & 20 & 0.0510 & 0.0570 & 0.0520 & 0.0545 & 0.0540 & 0.0490 & 0.0610 & 0.0575 & 0.0520 & 0.0435 & 0.0470 & 0.0550 & 0.1440 & 0.1190 & 0.1470 & 0.1245 & 0.1800 & 0.1630 \\
\hline & 100 & 0.0530 & 0.0575 & 0.0470 & 0.0420 & 0.0490 & 0.0515 & 0.0430 & 0.0555 & 0.0580 & 0.0515 & 0.0480 & 0.0520 & 0.1290 & 0.1165 & 0.1550 & 0.1280 & 0.1730 & 0.1470 \\
\hline
\end{tabular}

Table 0.1: Monte Carlo absolute bias, mean squared error and size, nominal size 5\%, dependent weight matrices regenerated in each trial as in Section . 